\title{
Sarcopenia in hospitalized geriatric patients
}

\author{
Citation for published version (APA):
}

Sipers, W. (2021). Sarcopenia in hospitalized geriatric patients: prevalence, diagnosis and consequences. [Doctoral Thesis, Maastricht University]. Zalsman Innovative Print. https://doi.org/10.26481/dis.20210115ws

Document status and date:

Published: 01/01/2021

DOI:

10.26481/dis.20210115ws

Document Version:

Publisher's PDF, also known as Version of record

\section{Please check the document version of this publication:}

- A submitted manuscript is the version of the article upon submission and before peer-review. There can be important differences between the submitted version and the official published version of record.

People interested in the research are advised to contact the author for the final version of the publication, or visit the DOI to the publisher's website.

- The final author version and the galley proof are versions of the publication after peer review.

- The final published version features the final layout of the paper including the volume, issue and page numbers.

Link to publication

\footnotetext{
General rights rights.

- You may freely distribute the URL identifying the publication in the public portal. please follow below link for the End User Agreement:

www.umlib.nl/taverne-license

Take down policy

If you believe that this document breaches copyright please contact us at:

repository@maastrichtuniversity.nl

providing details and we will investigate your claim.
}

Copyright and moral rights for the publications made accessible in the public portal are retained by the authors and/or other copyright owners and it is a condition of accessing publications that users recognise and abide by the legal requirements associated with these

- Users may download and print one copy of any publication from the public portal for the purpose of private study or research.

- You may not further distribute the material or use it for any profit-making activity or commercial gain

If the publication is distributed under the terms of Article $25 \mathrm{fa}$ of the Dutch Copyright Act, indicated by the "Taverne" license above, 


\section{Sarcopenia in hospitalized geriatric patients}

prevalence, diagnosis and consequences 
(c) Copyright Walther Sipers, Sittard 2021

All rights reserved. No part of this thesis may be reproduced, distributed, or transmitted in any form or by any means electronic or mechanical, including photocopying, recording, or any information storage or retrieval system, without permission from the author or when appropriate, from the publisher of the publications.

The studies presented in this thesis were performed at the Department of Geriatric Medicine of Zuyderland Medical Centre in collaboration with the Department of Human Biology, NUTRIM School of Nutrition and Translational Research in Metabolism, Faculty of Health, Medicine and Life Sciences and the Department of Health Services Research and the Department of Family Medicine Caphri - Care and Public Health Research Institute, Maastricht University.

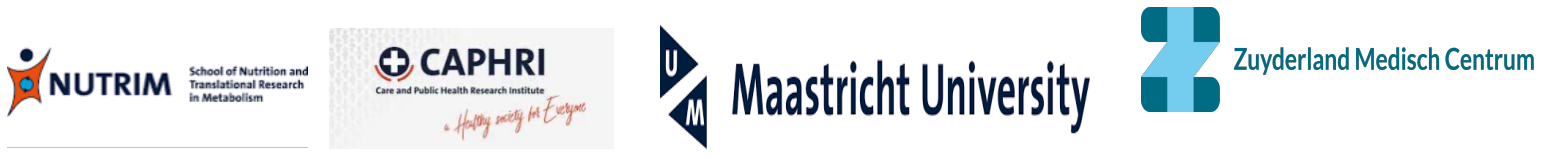

ISBN/EAN: 978-90-9034100-2

Cover design: Photo of Mr. Jussent (with permission) and the author

Printed by: Zalsman Innovative Print, Kampen

Printing of this thesis was financially supported by Zuyderland Medical Centre, Heerlen-Sittard-Geleen; Abbott B.V., Hoofddorp and Nutricia Specialized Nutrition, Zoetermeer

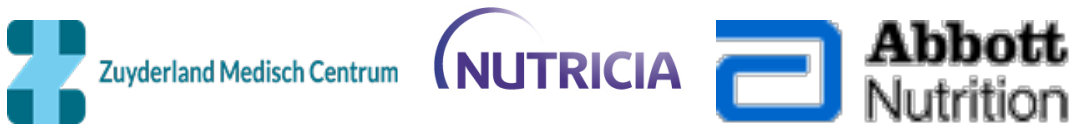




\title{
Sarcopenia in hospitalized geriatric patients
}

\author{
prevalence, diagnosis and consequences
}

\section{Dissertation}

To obtain the degree of Doctor at Maastricht University, on the authority of the Rector Magnificus, Prof. Dr. Rianne M. Letschert in accordance with the decision of the Board of Deans, to be defended in public on Friday 15 January 2021, at 10:00 hours

by

Wilhemus Maria Waltherus Hubertus Sipers

Born 13 March 1965, Maastricht 


\section{Promotor:}

Prof. dr. L.J.C. van Loon

Prof. dr. J.M.G.A. Schols

\section{Co-promotor:}

Dr. L.B. Verdijk

\section{Assessment Committee:}

Prof. dr. R.A. de Bie (chair)

Prof. dr. A.F. Lenssen

Prof. dr. A.M.W.J. Schols

Prof. dr. C.P.G.M. de Groot (Wageningen University \& Research, Wageningen)

Prof. dr. M.G.M. Olde Rikkert (Radboud Universiteit, Nijmegen) 


\section{Sarcopenia in hospitalized geriatric patients prevalence, diagnosis and consequences}

\section{Table of contents}

$\begin{array}{lll}\text { Chapter } 1 \quad \text { General introduction } & 7\end{array}$

Chapter 2 Impact of different diagnostic criteria on the prevalence of 17 sarcopenia in an acute care geriatric ward

Chapter 3 Sarcopenia is related to mortality in the acutely hospitalized geriatric patient

Chapter 4 Multi-frequency bioelectrical impedance analysis may represent a reproducible and practical tool to assess skeletal muscle mass in euvolemic acutely ill hospitalized geriatric patients

Chapter 5 The Martin Vigorimeter represents a reliable and more practical tool than the Jamar dynamometer to assess handgrip strength in the geriatric patient

Chapter $6 \quad$ General discussion

Valorisation

Dankwoord 



\section{CHAPTER 1}

General introduction 


\section{General introduction}

"Mister $T$ is an 83-year old man living independently with his wife. He has always loved to gardening. As a former mason, he had complained about low back pain for a number of years and habitual physical activity became more and more difficult. A year ago, he stopped his weekly walking with a friend and his son took over the gardening. Most of the time he was sitting in his chair, reading books and watching television. Recently, because of a pneumonia complicated with loss of mobility, he was admitted to a geriatric ward in the community hospital. The first days after hospitalization he was bedridden, and eating was a problem because he felt so tired and suffered from loss of appetite. He was treated by the multidisciplinary geriatric team and recovered soon from the pneumonia. However, mobilisation was complicated despite getting him on a chair as soon as possible in combination with daily exercise sessions by the ward physiotherapist. Therefore, he was transferred to the geriatric rehabilitation ward first, and finally returned home after 6 weeks. At home, however, he is not able to climb the stairs anymore, getting out of a chair remains challenging, and with a walker he can walk only 15 meters in his garden, after which he is completely exhausted. His wife helps dressing him and his bed is placed in the living room. Although Mr T is happy to be back home with his wife, he feels sorry about his functional losses, which hampers him to go back to leading his previous life."

Many geriatricians will recognise the scenario of $\mathrm{Mr} \mathrm{T}$ from their daily practice of acute care geriatric ward patients. This scenario raises many questions. Is sarcopenia a probable mechanism underlying the functional decline that is observed? How can we recognise patients with sarcopenia? And importantly, what can we do to stop the loss of physical functioning and potentially regain functional capacity in these patients? In this general introduction, the following issues related to these questions will be addressed: ageing, frailty, sarcopenia, and the acute geriatric ward patients. We end by shortly describing the outline of this dissertation.

\section{Aging}

Worldwide, the population is aging rapidly. In high-income countries such as the Netherlands, the continuing increase in life expectancy is mainly the result of a declining mortality among the oldest old (1). If the increase in life expectancy continues throughout the 21st century, most babies born since 2000 in Western Europe will be able to celebrate their 100th birthday (2). Some older people may complete marathons and even Ironman events, however, most older people will not be able to do so as they will suffer from diminished endurance as well as a decline in functional capacity. Aging is generally accompanied by physical and functional 
deterioration and can be considered a very complex phenomenon where interactions of many processes at several levels are involved (3). Homeostatic regulatory processes normally tend to keep the environment of the cells within relatively narrow bounds. On the other hand, the body is constantly adapting to several endogenous and exogenous stresses such as those induced by environmental factors, physical activity, and food consumption. At a biological level, molecular and cellular damage induced by such stresses will accumulate over time, leading to a general age-related decline of the individual health status (4). Despite this, the physiological changes that occur over time are only loosely associated with chronological age, and many other factors determine an individual's status at each phase throughout the lifespan. Importantly, old age itself does not define physical deterioration primarily. Some patients remain vigorous, despite an advanced age, while others show a gradual yet unrelenting functional decline in the absence of apparent disease states, or failure to recover following illness or hospitalization $(5,6)$, ultimately resulting in a condition of frailty.

\section{Physical frailty}

Frailty is a common and important geriatric syndrome characterized by age-associated declines in physiological reserve and function across multi-organ systems, leading to increased vulnerability for adverse health outcomes. Frail older adults are less able to adapt to stressors such as acute illness or trauma than younger or non-frail older adults (7). This increased vulnerability contributes to an increased risk of multiple adverse outcomes, including complications, falls, functional decline, institutionalization, disability, and death. Increasingly, frailty in older patients is considered to be the hallmark geriatric syndrome, and a forerunner to many other geriatric syndromes, including fractures, delirium, and incontinence. Two major frailty models have been described in the literature: the frailty phenotype, and the frailty index (8). The frailty phenotype defines frailty as a distinct clinical syndrome meeting three or more of five phenotypic criteria, also known as the Fried criteria: weakness, slowness, low level of physical activity, self-reported exhaustion, and unintentional weight loss. Given the nature of these criteria, this type of frailty can be considered as physical frailty (9). The frailty index on the other hand, defines frailty as cumulative deficits identified in a comprehensive geriatric assessment, thereby including the actual number of health deficits (e.g. symptoms, signs, laboratory abnormalities) that are counted in an individual, which is then divided by the total number of potential health deficits that were considered. This approach yields a number between 0 (no deficits) and 1 (all possible deficits). People with high frailty index scores are at a greatly increased risk of adverse outcomes, including death (10).

Significant progress has recently been made in understanding the pathogenesis of physical frailty. Chronic inflammation is likely a key pathophysiological process that contributes to the frailty syndrome directly and indirectly through other intermediate physiological systems, such 
as the musculoskeletal, endocrine, and haematological systems (6, 7). The complex multifactorial aetiologies of physical frailty also include obesity and specific diseases, such as e.g. chronic renal insufficiency and cardiovascular diseases. Despite controversies surrounding the best operational definition of frailty, there is a strong consensus among clinicians that frailty should be evaluated whenever feasible. Currently, nutrition, exercise and comprehensive geriatric interdisciplinary assessment and treatment are key interventions for physical frailty $(11,12)$. As our understanding of the biological basis and complexity of physical frailty further improves, more targeted preventive and counteractive interventional strategies, and innovative geriatric-care models will likely be developed $(8,12)$. In the context of clinical practice, physical frailty is a subclinical phenomenon and becomes visible due to an acute stressor, e.g. like a pneumonia in the case of $\mathrm{Mr} \mathrm{T}$, with the loss of mobility as one of the key characteristics. The loss of mobility is likely attributed to the age-related loss of muscle mass, quality and strength, called sarcopenia. Sarcopenia is more common in older adults and has been considered a precursor syndrome for frailty, as well as the physical manifestation of frailty (13).

\section{Sarcopenia}

The co-occurrence of low muscle mass and a decline in muscle function or strength has been termed sarcopenia, which is derived from the Greek words 'sarx' and 'penia', meaning 'flesh' and 'poverty' or 'lack'. Although Irwin Rosenberg first coined the term sarcopenia in 1989, it has taken some time for the European Working Group on Sarcopenia in Older People (EWGSOP) to develop an official (practical and clinical) definition (14). In their 2010 consensus paper, they describe sarcopenia as "a syndrome characterised by progressive and generalised loss of skeletal muscle mass and strength increasing the risk of frailty and predicting physical disability, loss of independence, poor quality of life and death" (14). In 2019, they revised the definition of sarcopenia and "reduced muscle strength" became the central hallmark of muscle failure, in combination with the loss of muscle mass or muscle quality (15). In some individuals, sarcopenia is largely attributed to ageing per se, whereas in many other cases, one or more specific causes can be identified. Thus, the categories of primary sarcopenia and secondary sarcopenia may be useful in clinical practice. Sarcopenia is considered 'primary' (or age-related) when no other specific causes are evident. Sarcopenia is considered 'secondary' when causal factors other than (or in addition to) ageing are evident, with multiple factors being mentioned in literature. For example, sarcopenia can occur secondary to a systemic disease, especially one that may invoke inflammatory processes, e.g. malignancy or organ failure. In addition, physical inactivity and disuse contribute to the development of sarcopenia, whether due to a sedentary lifestyle or to disease-related immobility or disability. Furthermore, sarcopenia may develop as a result of inadequate intake 
of energy or protein, which may be due to anorexia, malabsorption, and limited access to healthy foods, or a limited ability or reduced desire to eat. Apart from differentiating between primary and secondary sarcopenia, the EWGSOP identifies subcategories of sarcopenia as acute and chronic. Sarcopenia that has lasted less than 6 months is considered an acute condition, while sarcopenia lasting $\geq 6$ months is considered a chronic condition. Acute sarcopenia is usually related to an acute illness or injury, and, therefore, highly prevalent in the acute care geriatric wards, while chronic sarcopenia is likely associated with chronic and progressive conditions (15).

Muscle tissue can be considered as the engine of the human body. Cardiac muscle is the dominant tissue in the heart that is of vital importance by propelling oxygen and nutrients to our cells. Muscle is also found as smooth muscle tissue in the walls of our hollow organs, generating the driving force for propulsion of food and for the regulation of blood pressure. Finally, the mobility of the body as a whole reflects the activity of skeletal muscle tissue. The most important function of skeletal muscle for the body is the generation of force that is necessary for the production of movements, maintenance of posture, and stabilisation of joints, but it also plays a vital role in metabolism and the production of heat (16). Skeletal muscle makes up nearly half of the body's mass in healthy adult humans. In older people, the condition of skeletal muscle mass is of vital importance for their daily functional performance and overall health. However, one of the age-related changes that strongly impacts on health and function is a gradual, progressive loss of skeletal muscle mass. Adults lose an average of $25 \%$ of their muscle mass between the age of 40 and 70 years, with muscle loss accelerating after the age of 70 years, although with large inter-individual variation $(14,17)$. Age related loss of muscle mass and strength is characterized by a decline in muscle fibre size, with specific atrophy of type II muscle fibers, a decreased number of motor units, and accumulation of fat within muscle tissue $(18,19)$.

The decline in muscle mass with aging can be attributed to various processes. However, their relative contribution to the process of normal aging has not been well characterized and likely differs considerably between individuals. The balance between muscle protein synthesis and breakdown regulates muscle mass maintenance, and a negative net muscle protein balance is the common ground for any type of muscle loss. Since food (protein) intake and physical activity represent the two main anabolic stimuli, it is evident that both malnutrition and a reduction in physical activity play a key role in age-related muscle loss (20). In addition, genetic potential, hormonal changes, impairments in muscle autophagy and apoptosis, reduced mitochondrial content and function, inflammation, and loss of lower motor neurons can all play a role in tipping the balance towards a more negative net muscle protein balance and, thus, muscle loss in the older population $(18,21,22)$. Importantly, inflammation, 
malnutrition and prolonged bed rest often accompany hospitalisation of older people, and these factors strongly accelerate the rate of muscle loss. Hence, it is of particular interest to study skeletal muscle mass, function, and metabolism in frail and more clinically compromised older people admitted to hospital.

Because of the negative consequences of sarcopenia it is important to detect sarcopenia as early as possible, to understand the various factors contributing to its development, and to apply interventional strategies to attenuate or even prevent the loss of skeletal muscle mass in older individuals prior to and during hospitalisation. As the majority of studies on sarcopenia generally include healthy older subjects, there is a relative lack of data on the prevalence and incidence of sarcopenia in frail old and hospitalized people, as well as on the impact of nutrition and exercise interventions to attenuate or prevent the loss of muscle mass and strength in this target group (23-25).

\section{The hospitalized geriatric patient}

The (acutely) hospitalized geriatric patient is typically severely frail, showing a profile of multimorbidity, polypharmacy, and disability (26). About 30 percent of hospitalized older patients show frequent and severe disease presentations, a higher risk of iatrogenic injury during hospitalization, functional decline, loss of mobility and self-care abilities during the course of an acute medical illness (27-29). In hospitalized geriatric patients, functional decline and loss of mobility are likely attributed to pre-existing sarcopenia, either: primary, secondary, chronic or acute, or a combination of these categories (15). In acute care geriatric wards, care is designed to prevent patients' loss of independence in the performance of daily activities needed for autonomous living (hospital associated disability) from admission to discharge (29). Both acute care geriatric wards or acute care for elders units interventions include principles of a prepared environment that encourages safe patient self-care, a set of clinical guidelines for bedside care by nurses and other health professionals to prevent patient disability and restore self-care lost by the acute illness, and planning for transitions of care and medical care (30).

By applying a structural care approach, an interdisciplinary geriatric team completes a geriatric assessment, follows clinical guidelines, and initiates plans for care transitions together with the patient and their family members. This strategy has already been proven successful in terms of a reduced functional disability among patients, reduced risk of nursing home admission and lower costs of hospitalization (30-32).

Nevertheless, despite this strategy with multidisciplinary interventions, many older patients show a substantial decline in physical function following hospitalization (33), as also illustrated in Mr. T's case at the start of this introduction. In hospitalized geriatric patients, frailty, cachexia, and sarcopenia may coexist and it is difficult to unravel these syndromes as they overlap. Sarcopenia is probably an underlying explanation for the functional decline (34). 
Sarcopenia is highly prevalent $(21-28 \%)$ in the hospitalized older patient and this number is probably even higher in the acute care geriatric wards. Sarcopenia has been associated with adverse outcomes despite multi-disciplinary interventions (35-37). As such, recognizing sarcopenia in hospitalized geriatric patients should be the starting point in preventing adverse outcomes. Currently though, screening for sarcopenia is not part of the comprehensive geriatric assessment in daily clinical practice of acute care geriatric wards (38).

The challenge for clinical practice, therefore will be the assessment of sarcopenia to identify those who might benefit most from interventions that attenuate or prevent further loss of muscle mass, strength, and/or functional capacity. However, as we often lack detailed information on the functional status of patients prior to admission to the ward, we are unaware how many patients like Mr. $\mathrm{T}$ are admitted to the acute care geriatric hospital ward. Furthermore, we can only speculate on the impact of pre-existing sarcopenia on the clinical and functional outcome in these patients.

Despite a relative large number of methods and tools available to assess muscle mass, muscle strength, and physical performance, there is a lack of knowledge on the applicability and reliability of these methods in this specific population. Many of the accepted methods are not feasible for the assessment of sarcopenia in practice in hospitalized geriatric patients because of e.g. comorbid diseases or low physical capacity. Furthermore, the clinical condition of acutely admitted geriatric patients is not stable and changes throughout their hospitalization, which further compromises the accurate assessment of muscle mass, strength, or functional performance.

\section{Dissertation outline}

This dissertation describes several studies performed in the acute geriatric ward of a general hospital to provide further insight into the assessment, prevalence and clinical outcomes of sarcopenia-associated issues in this clinical care setting. First, the impact of different diagnostic criteria for sarcopenia on the prevalence of sarcopenia in acutely hospitalized geriatric patients is presented in Chapter 2. Next, we examined whether sarcopenia is associated with mortality in this population. Although there is no consensus about the exact definition and criteria for sarcopenia, it is generally acknowledged that sarcopenia is related to adverse outcome in different populations. However information about the impact of sarcopenia in acutely ill hospitalized geriatric patients on outcome is sparse. In chapter $\mathbf{3}$ we examine the relation of different criteria for sarcopenia with mortality in acutely hospitalized geriatric patients. Here, we also address which hallmarks of sarcopenia and/or other patients' characteristics represent the strongest predictors of 1 and 2-year mortality in these geriatric patients. Thereafter, the attention is shifted to the actual measurement of different sarcopenia characteristics in the clinical setting. As pointed out, measuring hallmarks of sarcopenia like 
skeletal muscle mass and muscle strength may be difficult in acutely ill geriatric patients because of functional limitations, multimorbidity, and multimodal treatment. In chapter 4, we assessed the reproducibility of measuring skeletal muscle mass with bio-impedance analysis (BIA), as this represents a mobile, non-invasive, inexpensive, and easy to use measurement. Specific attention is paid to the impact of an abnormal hydration status on the reproducibility of measuring muscle mass with BIA. Though much attention has gone out to the amount of muscle mass, muscle strength and functional capacity are likely of more relevance to the patient. In chapter 5 , we describe a study where we assessed handgrip strength as a proxy of overall muscle strength, and a hallmark of sarcopenia. The applicability and test-retest reliability of measuring handgrip strength with two different handheld dynamometers, the Martin Vigorimeter and the Jamar dynamometer are compared in geriatric patients during hospitalization. The general discussion is delineated in Chapter 6, where the overall findings of this thesis and their implications for daily practice are discussed. Furthermore, a few key areas for further research are identified. 


\section{References}

1. Christensen K, Doblhammer G, Rau R, Vaupel JW. Ageing populations: the challenges ahead. Lancet. 2009;374(9696):1196-208.

2. Vaupel JW. Biodemography of human ageing. Nature. 2010;464(7288):536-42.

3. da Costa JP, Vitorino R, Silva GM, Vogel C, Duarte AC, Rocha-Santos T. A synopsis on aging-Theories, mechanisms and future prospects. Ageing Res Rev. 2016;29:90112.

4. Hanson MA, Cooper C, Aihie Sayer A, Eendebak RJ, Clough GF, Beard JR. Developmental aspects of a life course approach to healthy ageing. J Physiol. 2016;594(8):2147-60.

5. Ding YY, Kuha J, Murphy M. Pathways from physical frailty to activity limitation in older people: Identifying moderators and mediators in the English Longitudinal Study of Ageing. Exp Gerontol. 2017;98:169-76.

6. Ferrucci L, Fabbri E. Inflammageing: chronic inflammation in ageing, cardiovascular disease, and frailty. Nat Rev Cardiol. 2018;15(9):505-22.

7. Cornman JC, Glei DA, Goldman N, Weinstein M. Physiological Dysregulation, Frailty, and Risk of Mortality Among Older Adults. Res Aging. 2017;39(8):911-33.

8. Walston J, Buta B, Xue QL. Frailty Screening and Interventions: Considerations for Clinical Practice. Clin Geriatr Med. 2018;34(1):25-38.

9. Fried LP, Tangen CM, Walston J, Newman AB, Hirsch C, Gottdiener J, et al. Frailty in older adults: evidence for a phenotype. J Gerontol A Biol Sci Med Sci. 2001;56(3):M146-56.

10. Rockwood K, Blodgett JM, Theou O, Sun MH, Feridooni HA, Mitnitski A, et al. A Frailty Index Based On Deficit Accumulation Quantifies Mortality Risk in Humans and in Mice. Sci Rep. 2017;7:43068.

11. Hoogendijk EO, Afilalo J, Ensrud KE, Kowal P, Onder G, Fried LP. Frailty: implications for clinical practice and public health. Lancet. 2019;394(10206):1365-75.

12. Dent E, Morley JE, Cruz-Jentoft AJ, Woodhouse L, Rodriguez-Manas L, Fried LP, et al. Physical Frailty: ICFSR International Clinical Practice Guidelines for Identification and Management. J Nutr Health Aging. 2019;23(9):771-87.

13. Wilson D, Jackson T, Sapey E, Lord JM. Frailty and sarcopenia: The potential role of an aged immune system. Ageing Res Rev. 2017;36:1-10.

14. Cruz-Jentoft AJ, Baeyens JP, Bauer JM, Boirie Y, Cederholm T, Landi F, et al. Sarcopenia: European consensus on definition and diagnosis: Report of the European Working Group on Sarcopenia in Older People. Age Ageing. 2010;39(4):412-23.

15. Cruz-Jentoft AJ, Bahat G, Bauer J, Boirie Y, Bruyere O, Cederholm T, et al. Sarcopenia: revised European consensus on definition and diagnosis. Age Ageing. 2019;48(1):16-31.

16. Murphy AC, Muldoon SF, Baker D, Lastowka A, Bennett B, Yang M, et al. Structure, function, and control of the human musculoskeletal network. PLoS Biol. 2018;16(1):e2002811.

17. McCormick R, Vasilaki A. Age-related changes in skeletal muscle: changes to life-style as a therapy. Biogerontology. 2018;19(6):519-36.

18. Wilkinson DJ, Piasecki M, Atherton PJ. The age-related loss of skeletal muscle mass and function: Measurement and physiology of muscle fibre atrophy and muscle fibre loss in humans. Ageing Res Rev. 2018;47:123-32.

19. Larsson L, Degens H, Li M, Salviati L, Lee YI, Thompson W, et al. Sarcopenia: AgingRelated Loss of Muscle Mass and Function. Physiol Rev. 2019;99(1):427-511.

20. Kraemer RR, Castracane VD. Novel insights regarding mechanisms for treatment of sarcopenia. Metabolism. 2015;64(2):160-2.

21. Ali S, Garcia JM. Sarcopenia, cachexia and aging: diagnosis, mechanisms and therapeutic options - a mini-review. Gerontology. 2014;60(4):294-305. 
22. Beaudart C, Dawson A, Shaw SC, Harvey NC, Kanis JA, Binkley N, et al. Nutrition and physical activity in the prevention and treatment of sarcopenia: systematic review. Osteoporos Int. 2017;28(6):1817-33.

23. Luo D, Lin Z, Li S, Liu SJ. Effect of nutritional supplement combined with exercise intervention on sarcopenia in the elderly: A meta-analysis. Int $\mathrm{J}$ Nurs Sci. 2017;4(4):389-401.

24. Robinson SM, Reginster JY, Rizzoli R, Shaw SC, Kanis JA, Bautmans I, et al. Does nutrition play a role in the prevention and management of sarcopenia? Clin Nutr. 2018;37(4):1121-32.

25. Tessier AJ, Chevalier S. An Update on Protein, Leucine, Omega-3 Fatty Acids, and Vitamin $D$ in the Prevention and Treatment of Sarcopenia and Functional Decline. Nutrients. 2018;10(8).

26. Bell SP, Vasilevskis EE, Saraf AA, Jacobsen JM, Kripalani S, Mixon AS, et al. Geriatric Syndromes in Hospitalized Older Adults Discharged to Skilled Nursing Facilities. J Am Geriatr Soc. 2016;64(4):715-22.

27. Brummel NE, Balas MC, Morandi A, Ferrante LE, Gill TM, Ely EW. Understanding and reducing disability in older adults following critical illness. Crit Care Med. 2015;43(6):1265-75.

28. Gill TM, Allore HG, Holford TR, Guo Z. Hospitalization, restricted activity, and the development of disability among older persons. JAMA. 2004;292(17):2115-24.

29. Covinsky KE, Pierluissi E, Johnston CB. Hospitalization-associated disability: "She was probably able to ambulate, but I'm not sure". JAMA. 2011;306(16):1782-93.

30. Ahlund $\mathrm{K}$, Back $\mathrm{M}$, Oberg $\mathrm{B}$, Ekerstad $\mathrm{N}$. Effects of comprehensive geriatric assessment on physical fitness in an acute medical setting for frail elderly patients. Clin Interv Aging. 2017;12:1929-39.

31. Ekerstad N, Karlson BW, Dahlin Ivanoff S, Landahl S, Andersson D, Heintz E, et al. Is the acute care of frail elderly patients in a comprehensive geriatric assessment unit superior to conventional acute medical care? Clin Interv Aging. 2017;12:1-9.

32. Ekerstad N, Dahlin Ivanoff S, Landahl S, Ostberg G, Johansson M, Andersson D, et al. Acute care of severely frail elderly patients in a CGA-unit is associated with less functional decline than conventional acute care. Clin Interv Aging. 2017;12:1239-49.

33. Zhao Y, Zhang Y, Hao Q, Ge M, Dong B. Sarcopenia and hospital-related outcomes in the old people: a systematic review and meta-analysis. Aging Clin Exp Res. 2019;31(1):5-14.

34. Beaudart C, Zaaria M, Pasleau F, Reginster JY, Bruyere O. Health Outcomes of Sarcopenia: A Systematic Review and Meta-Analysis. PLoS One. 2017;12(1):e0169548.

35. Sousa AS, Guerra RS, Fonseca I, Pichel F, Amaral TF. Sarcopenia among hospitalized patients - A cross-sectional study. Clin Nutr. 2015;34(6):1239-44.

36. Cerri AP, Bellelli G, Mazzone A, Pittella F, Landi F, Zambon A, et al. Sarcopenia and malnutrition in acutely ill hospitalized elderly: Prevalence and outcomes. Clin Nutr. 2015;34(4):745-51.

37. Vetrano DL, Landi F, Volpato S, Corsonello A, Meloni E, Bernabei R, et al. Association of sarcopenia with short- and long-term mortality in older adults admitted to acute care wards: results from the CRIME study. J Gerontol A Biol Sci Med Sci. 2014;69(9):115461.

38. Parker SG, McCue P, Phelps K, McCleod A, Arora S, Nockels K, et al. What is Comprehensive Geriatric Assessment (CGA)? An umbrella review. Age Ageing. 2018;47(1):149-55. 


\title{
CHAPTER 2
}

\section{Impact of different diagnostic criteria on the}

\author{
prevalence of sarcopenia in an acute care
}

\author{
geriatric ward
}

Key words: sarcopenia, diagnostic criteria, prevalence, hospitalized geriatric patients

Walther Sipers, Judith Meijers, Ruud Halfens, Rian Bibo, Jos Schols Journal of Frailty \& Aging. 2014;3(4):222-9. 


\begin{abstract}
Background: Sarcopenia is probably an important causal factor for functional decline in acutely ill hospitalized geriatric patients. Low skeletal muscle mass, low gait speed and low grip strength are hallmarks of diagnosing sarcopenia. However there are many different diagnostic criteria to assess sarcopenia.
\end{abstract}

Objectives: In this study the influence of different criteria for sarcopenia was studied on sarcopenia prevalence in geriatric patients admitted to an acute care hospital.

Design: Cross sectional study design.

Setting: A geriatric ward of a large Dutch hospital.

Participants: Geriatric patients.

Measurements: Skeletal muscle mass measured using bio impedance analysis (BIA), gait speed using the 4 meter walking test and grip strength. The sarcopenia prevalence was investigated according to criteria of: muscle mass, grip strength, the European Working Group on Sarcopenia in Elderly People, the International Working Group on Sarcopenia and the Special Interest Group of Society of Sarcopenia, Cachexia and Wasting Disorders.

Results: 85 geriatric patients were included (61 women). Applying the 17 different criteria, the sarcopenia prevalence varied from $26-75 \%$ for women and from $42-100 \%$ for men. Comparing the Janssen calculation with the Maltron calculation sarcopenia prevalence ranged from respectively $26-67 \%$ and $67-70 \%$ for women and from $42-71 \%$ and $75-100 \%$ for men. Almost all patients $(96 \%)$ had a low gait speed.

Conclusions: Sarcopenia is highly prevalent in an acute hospitalized geriatric population, although the prevalence varies widely depending on the diagnostic criteria applied. A prospective study is needed to discover which criteria of sarcopenia can predict best adverse outcomes. 


\section{Introduction}

Acute hospitalization is a hazardous event with a high mortality rate for older people and high rates of post-discharge disability (1). This is especially the case for geriatric patients, the frailest elderly people $(1,2)$. Accelerated muscle loss due to acute illness, bed rest and malnutrition, combined with a decline in physical performance, is probably an important causal factor for functional decline and adverse outcomes $(3,4,5)$. Reduced muscle strength has been found to be associated with dependency in daily living activities and with mortality $(6,7,8)$.

It was Rosenberg, in 1989, who first described this loss of muscle mass with aging as sarcopenia (9). It was more than another 10 years before the European Working Group on Sarcopenia in Elderly People (EWGSOP) developed the first diagnostic criteria with a diagnostic algorithm (10). This group indicated that muscle strength, physical performance and muscle mass have to be determined to assess sarcopenia. In addition, the International Working Group on Sarcopenia (IWGS) and Special Interest Group (SIG) of the Society of Sarcopenia, Cachexia and Wasting Disorders developed additional definitions and criteria for sarcopenia $(11,12)$. The IWGS defined sarcopenia as the loss of muscle mass with a physical performance measure (6). The SIG defined sarcopenia with limited mobility if a patient had low muscle mass and low physical performance (12). The debate about the definition of sarcopenia continues and there is still no consensus.

In addition to various definitions, there are different methods with several techniques, equations, reference populations and devices for measuring different domains of sarcopenia such as muscle mass, gait speed and grip strength (10). For example, when applying Bioelectrical Impedance Analysis (BIA), there are devices with single-frequency or multifrequency multi-segmental measurement and with different BIA calculations $(13,14)$.

Although sarcopenia seems to be an important and prevalent clinical topic in geriatric medicine, to our knowledge there are only a few publications specifically about geriatric patients in acute hospital geriatric wards. Rossi and colleagues found a prevalence of $26 \%$ for sarcopenia among acutely ill persons in a geriatric unit in an academic medical hospital (15). Other researchers found a prevalence of $25.3 \%$ in an acute geriatric ward of a general hospital (16). A prevalence of $28 \%$ was found in a cohort from the CRIME project in geriatric and internal medicine acute care wards at seven Italian hospitals (17).

In this study, we investigated the prevalence of sarcopenia according to skeletal muscle mass with BIA using different calculations related to height and weight. We applied the EWGSOP, IWGS and SIG criteria to geriatric patients admitted to an acute care hospital $(10,11,12)$. Our research questions were:

- What is the prevalence of sarcopenia according to skeletal muscle mass, EWGSOP, IWGS and SIG criteria among geriatric patients admitted to a geriatric ward of an acute care hospital? 
- What is the influence of different BIA equations, Maltron or Janssen calculation, on the prevalence of sarcopenia in the geriatric patients?

- How many patients are sarcopenic according to all the definitions tested in this study?

\section{Methods}

Design

This study followed a cross-sectional design.

\section{Study sample}

All geriatric patients admitted to the acute geriatric ward of a large Dutch general hospital were included in the study. For organizational reasons, there were two periods of recruitment: the first from March through June 2012 and the second for the same months in 2013. The inclusion criteria were an age above 70 years, the ability to walk prior to admission and being physical frail according to the Fried criteria (18).

Patients were excluded if they had a pacemaker or an implantable cardioverter defibrillator (ICD), were not able to perform instructions because of a severe confusional state or dementia, or had a terminal condition. Patients were also excluded if they were transferred from another hospital department or if there was no informed consent by patient or proxy.

\section{Measurements}

\section{Patient characteristics}

Patient characteristics were retrieved from the medical and nursing files. These included sex, age, living situation, diagnosed medical conditions, medical history and activities of daily living prior to the acute illness that led to hospital admission. Height was estimated to the nearest centimeter by measuring ulna length because many patients were temporarily bedridden (19). The physical frailty score was assessed according to the Fried criteria, which ranges from 0 to 5: a score of 3 or higher indicates physical frailty (18). Weight was measured on a sitting weight scale (SECA, Model 959). Measurements of handgrip strength, muscle mass and gait speed were made on the fourth day after hospital admission.

A number of scales were used to evaluate patient characteristics. The cumulative illness rating scale (CIRS) was used to calculate the number and severity of chronic illnesses of the patients' comorbid diseases. The score ranges from 0 , which corresponds to the absence of disorders, to a maximum of 56 (20). Malnutrition was measured using the Short Nutritional Assessment Questionnaire (SNAQ), which is a validated screening instrument for malnutrition. Scores range from 0 to 5 ; a score of 3 or higher indicates that the patient is malnourished (21). 
The Katz ADL-6, a validated instrument for screening daily living activities, was used to assess ADL. Scores range from 0 (totally independent) to 6 (completely dependent) (22).

\section{Handgrip strength}

Handgrip strength was assessed using the Jamar dynamometer (Sammons Preston, Inc., Bolingbrook, IL, USA), a frequently used and validated tool for assessing healthy elderly people. We applied the Southampton protocol, which is described in detail in the review by Roberts et al $(2011)(23,24)$.

\section{Muscle mass}

The Maltron BioScan 920-II, a multi-frequency multi-segmental bio-impedance (mf-ms BIA) device, was used to measure muscle mass. Mf-ms BIA has been validated for the assessment of whole body composition and segmental lean mass in elderly people $(6,25)$. The Maltron BioScan 920-II has an eight-point electrode system, which separately measures impedance of the patient's trunk, arms and legs at four different frequencies $(5 \mathrm{kHz}, 50 \mathrm{kHz} 100 \mathrm{~Hz}$ and 200 $\mathrm{Hz}$ ) for each body segment. The Maltron BioScan 920-II calculates appendicular skeletal muscle mass according to the Janssen calculation and a device-specific calculation called the Maltron calculation (25). Patients were measured early in the morning before breakfast, wearing only the pajamas described in the user's manual.

\section{Gait speed}

The four-meter walking test has been validated for measuring gait speed in elderly people. The faster of two trials was used and the test was started from a standing still position. Patients were instructed to walk at an easy usual speed and were allowed to use a walking aid if necessary (26).

\section{Diagnostic criteria for sarcopenia}

Table 1 shows the different diagnostic criteria for sarcopenia that are described in the literature and were used as reference criteria in this study. They are based on measurements of muscle mass by BIA, handgrip strength and gait speed.

\section{Statistics}

Data were analyzed using IBM SPSS Statistics 20. Due to the aim of the study, descriptive statistics were used. As the prevalence of sarcopenia is different for men and women, data were analyzed for men and woman separately (27). Categorical data are presented as 
frequencies and percentages, and discrete and continuous data are presented as means with standard deviations.

The Ethics committee of Sittard-Heerlen approved the study (number 13-N-60) and participants signed an informed consent.

\section{Results}

In the two three-month periods, 205 older patients were admitted and asked to participate. At baseline, 77 patients were excluded. Of that number, 13 patients or proxies refused to participate. Another 64 patients were excluded because they did not meet the inclusion criteria: 10 patients had a severe confusional state or dementia, 7 had a pacemaker or ICD, 13 were unable to walk, 24 had a terminal condition and 10 were transferred from another hospital department.

After exclusion, 128 patients were eligible to participate. However, there was an incomplete data set for 34 patients and there were technical problems with the BIA or Jamar dynamometer when measuring 9 patients. In the end, 85 patients with complete datasets were included in the analyses.

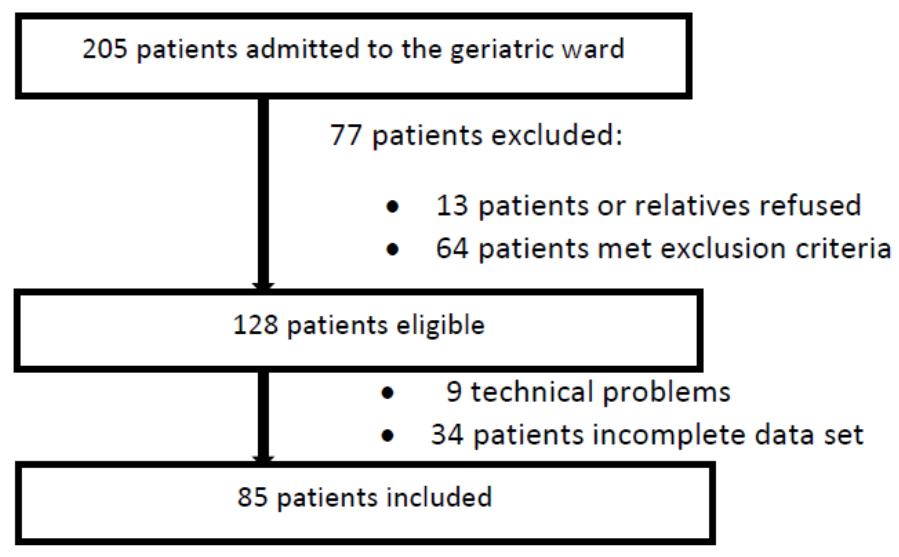

Figure 1: Flow chart describing patient recruitment.

The mean age of participants was 84 years and $72 \%$ were female. The mean Fried score was 3.8 for men $(S D=0.7)$ and 4.0 for women $(S D=0.7)$. Sixty-nine percent of the women and $92 \%$ of the men lived in the community surrounding the hospital. The mean CIRS score was 19.3 $(S D=5.1)$ for women and $20.9(S D=5.7)$ for men. Thirty-nine percent of the participants were malnourished, with SNAQ scores of 3 or higher. Forty-seven percent were highly ADL dependent, with a Katz ADL-6 score of 5. 
Table 1: Cut-off points for sarcopenia based on different definitions

\begin{tabular}{|c|c|c|c|c|c|c|}
\hline Criterion & Code & Formula & Measurement & $\begin{array}{l}\text { Cut-off point, } \\
\text { sarcopenia } \\
\text { present }\end{array}$ & $\begin{array}{l}\text { Cut-off } \\
\text { for men }\end{array}$ & $\begin{array}{l}\text { Cut-off } \\
\text { for women }\end{array}$ \\
\hline \multirow[t]{4}{*}{$\begin{array}{l}\text { Muscle } \\
\text { mass }\end{array}$} & $\mathrm{A} 1$ & $\begin{array}{l}\text { Skeletal lean mass/body mass } x \\
100 \%\left(\mathrm{RMM}^{\star}\right)^{13,25}\end{array}$ & $\begin{array}{l}\text { Maltron Bioscan 920-II with } \\
\text { Maltron calculation }\end{array}$ & $\begin{array}{l}\text { Class I } \\
\text { Class II }\end{array}$ & $\begin{array}{l}37 \% \\
31 \%\end{array}$ & $\begin{array}{l}28 \% \\
22 \%\end{array}$ \\
\hline & A2 & $\mathrm{RMM}^{13}$ & $\begin{array}{l}\text { Maltron Bioscan 920-II with } \\
\text { Janssen calculation }\end{array}$ & $\begin{array}{l}\text { Class I } \\
\text { Class II }\end{array}$ & $\begin{array}{l}37 \% \\
31 \% \\
\end{array}$ & $\begin{array}{l}28 \% \\
22 \% \\
\end{array}$ \\
\hline & A3 & $\begin{array}{l}\text { Skeletal lean mass/ height }{ }^{2} \\
\left(\mathrm{SMI}^{\dagger}\right)^{7,25}\end{array}$ & $\begin{array}{l}\text { Maltron Bioscan 920-II with } \\
\text { Maltron calculation }\end{array}$ & $\begin{array}{l}\text { Moderate } \\
\text { Severe }\end{array}$ & $\begin{array}{l}10.75 \\
8.50 \mathrm{~kg} / \mathrm{m}^{2}\end{array}$ & $\begin{array}{l}6.75 \\
5.75 \mathrm{~kg} / \mathrm{m}^{2}\end{array}$ \\
\hline & A4 & $\mathrm{SMI}^{1,13}$ & $\begin{array}{l}\text { Maltron Bioscan 920-II with } \\
\text { Janssen calculation }\end{array}$ & $\begin{array}{l}\text { Moderate } \\
\text { Severe }\end{array}$ & $\begin{array}{l}10.75 \\
8.50 \mathrm{~kg} / \mathrm{m}^{2}\end{array}$ & $\begin{array}{l}6.75 \\
5.75 \mathrm{~kg} / \mathrm{m}^{2}\end{array}$ \\
\hline $\begin{array}{l}\text { Muscle } \\
\text { strength }\end{array}$ & $\mathrm{B}$ & $\begin{array}{l}\text { Grip strength below cut-off } \\
\text { point }^{10}\end{array}$ & Jamar dynamometer & Sarcopenia & $<30,3 \mathrm{~kg}$ & $<19,3 \mathrm{~kg}$ \\
\hline \multirow[t]{6}{*}{$\begin{array}{l}\text { Muscle } \\
\text { mass } \\
\text { and } \\
\text { gait speed } \\
\end{array}$} & $\mathrm{C} 1$ & $\begin{array}{l}\text { IWGS } \\
\text { Gait speed }<1 \mathrm{~m} / \mathrm{s} \text { and } \\
\text { SMI below cut-off point }{ }^{11,25}\end{array}$ & $\begin{array}{l}\text { 4-meter walking test } \\
\text { Maltron Bioscan 920-II with } \\
\text { Maltron calculation }\end{array}$ & $\begin{array}{l}\text { Moderate } \\
\text { Severe }\end{array}$ & $\begin{array}{l}<1 \mathrm{~m} / \mathrm{s}+ \\
10.75 \\
8.50 \mathrm{~kg} / \mathrm{m}^{2}\end{array}$ & $\begin{array}{l}<1 \mathrm{~m} / \mathrm{s}+ \\
6.75 \\
5.75 \mathrm{~kg} / \mathrm{m}^{2}\end{array}$ \\
\hline & $\mathrm{C} 2$ & $\begin{array}{l}\text { IWGS } \\
\text { Gait speed }<1 \mathrm{~m} / \mathrm{s} \text { and } \\
\text { SMI below cut-off point }\end{array}$ & $\begin{array}{l}\text { 4-meter walking test } \\
\text { Maltron Bioscan 920-II with } \\
\text { Janssen calculation }\end{array}$ & $\begin{array}{l}\text { Moderate } \\
\text { Severe }\end{array}$ & $\begin{array}{l}<1 \mathrm{~m} / \mathrm{s}+ \\
10.75 \\
8.50 \mathrm{~kg} / \mathrm{m}^{2}\end{array}$ & $\begin{array}{l}<1 \mathrm{~m} / \mathrm{s}+ \\
6.75 \\
5.75 \mathrm{~kg} / \mathrm{m}^{2}\end{array}$ \\
\hline & C3 & $\begin{array}{l}\text { IWGS } \\
\text { Gait speed }<1 \mathrm{~m} / \mathrm{s} \text { and } \\
\text { RMM below cut-off point } 11,25\end{array}$ & $\begin{array}{l}\text { 4-meter walking test } \\
\text { Maltron Bioscan 920-II with } \\
\text { Maltron calculation }\end{array}$ & $\begin{array}{l}\text { Class } 1 \\
\text { Class } 2\end{array}$ & $\begin{array}{l}<1 \mathrm{~m} / \mathrm{s}+ \\
37 \% \\
31 \%\end{array}$ & $\begin{array}{l}<1 \mathrm{~m} / \mathrm{s}+ \\
28 \% \\
22 \%\end{array}$ \\
\hline & $\mathrm{C} 4$ & $\begin{array}{l}\text { IWGS } \\
\text { Gait speed }<1 \mathrm{~m} / \mathrm{s} \text { and } \\
\text { RMM below cut-off point } 3,11\end{array}$ & $\begin{array}{l}\text { 4-meter walking test } \\
\text { Maltron Bioscan 920-II with } \\
\text { Janssen calculation }\end{array}$ & $\begin{array}{l}\text { Class } 1 \\
\text { Class } 2\end{array}$ & $\begin{array}{l}<1 \mathrm{~m} / \mathrm{s}+ \\
37 \% \\
31 \%\end{array}$ & $\begin{array}{l}<1 \mathrm{~m} / \mathrm{s}+ \\
28 \% \\
22 \%\end{array}$ \\
\hline & $\mathrm{C5}$ & $\begin{array}{l}\text { SIG } \\
\text { Gait speed }<0.8 \mathrm{~m} / \mathrm{s} \text { and } \\
\text { RMM }^{12,25}\end{array}$ & $\begin{array}{l}\text { 4-meter walking test } \\
\text { Maltron Bioscan 920-II with } \\
\text { Maltron calculation }\end{array}$ & $\begin{array}{l}\text { Class } 1 \\
\text { Class } 2 \\
\end{array}$ & $\begin{array}{l}<0.8 \mathrm{~m} / \mathrm{s}+ \\
37 \% \\
31 \%\end{array}$ & $\begin{array}{l}<0.8 \mathrm{~m} / \mathrm{s}+ \\
28 \% \\
22 \%\end{array}$ \\
\hline & C6 & $\begin{array}{l}\text { SIG } \\
\text { Gait speed }<0.8 \mathrm{~m} / \mathrm{s} \\
\text { and } \mathrm{RMM}^{3,12}\end{array}$ & $\begin{array}{l}\text { 4-meter walking test } \\
\text { Maltron Bioscan 920-II with } \\
\text { Janssen calculation }\end{array}$ & $\begin{array}{l}\text { Class } 1 \\
\text { Class } 2 \\
\end{array}$ & $\begin{array}{l}<0.8 \mathrm{~m} / \mathrm{s}+ \\
37 \% \\
31 \%\end{array}$ & $\begin{array}{l}<0.8 \mathrm{~m} / \mathrm{s}+ \\
28 \% \\
22 \%\end{array}$ \\
\hline \multirow[t]{6}{*}{$\begin{array}{l}\text { Muscle } \\
\text { mass } \\
\text { and } \\
\text { gait speed } \\
\text { and } \\
\text { grip } \\
\text { strength }\end{array}$} & D1 & $\begin{array}{l}\text { EWGSOP } \\
\text { Gait speed } \leq 0.8 \mathrm{~m} / \mathrm{s} \text { and } \\
\text { RMM below cut-off point } \\
\text { or } \\
\text { Gait speed }>0.8 \mathrm{~m} / \mathrm{s} \text { and } \\
\text { Grip strength and RMM below } \\
\text { cut-off points }\end{array}$ & $\begin{array}{l}\text { 4-meter walking test } \\
\text { Maltron Bioscan 920-II with } \\
\text { Maltron calculation } \\
\text { Jamar dynamometer }\end{array}$ & $\begin{array}{l}\text { Class } 1 \\
\text { Class } 2 \\
\\
\\
\text { Class } 1 \\
\text { Class } 2 \\
\end{array}$ & $\begin{array}{l}\leq 0.8 \mathrm{~m} / \mathrm{s}+ \\
37 \% \\
31 \% \\
\\
>0.8 \mathrm{~m} / \mathrm{s}+ \\
<30 \mathrm{~kg}+ \\
37 \% \\
31 \% \\
\end{array}$ & $\begin{array}{l}\leq 0.8 \mathrm{~m} / \mathrm{s}+ \\
28 \% \\
22 \% \\
>0.8 \mathrm{~m} / \mathrm{s}+ \\
<30 \mathrm{~kg}+ \\
28 \% \\
22 \%\end{array}$ \\
\hline & D2 & $\begin{array}{l}\text { EWGSOP } \\
\text { Gait speed } \leq 0.8 \mathrm{~m} / \mathrm{s} \text { and } \\
\text { RMM below cut-off point } \\
\text { or } \\
\text { Gait speed }>0.8 \mathrm{~m} / \mathrm{s} \text { and } \\
\text { Grip strength and RMM below } \\
\text { cut-off points, }\end{array}$ & $\begin{array}{l}\text { 4-meter walking test } \\
\text { Maltron Bioscan 920-II with } \\
\text { Janssen Calculation } \\
\text { Jamar dynamometer }\end{array}$ & $\begin{array}{l}\text { Class } 1 \\
\text { Class } 2 \\
\\
\\
\text { Class } 1 \\
\text { Class } 2 \\
\end{array}$ & $\begin{array}{l}\leq 0.8 \mathrm{~m} / \mathrm{s}+ \\
37 \% \\
31 \% \\
\\
>0.8 \mathrm{~m} / \mathrm{s}+ \\
<30 \mathrm{~kg}+ \\
37 \% \\
31 \% \\
\end{array}$ & $\begin{array}{l}\leq 0.8 \mathrm{~m} / \mathrm{s}+ \\
28 \% \\
22 \% \\
\\
>0.8 \mathrm{~m} / \mathrm{s}+ \\
<30 \mathrm{~kg}+ \\
28 \% \\
22 \%\end{array}$ \\
\hline & D3 & $\begin{array}{l}\text { EWGSOP } \\
\text { Gait speed } \leq 0.8 \mathrm{~m} / \mathrm{s} \text { and } \\
\text { RMM below cut-off point } \\
\text { or } \\
\text { Gait speed }>0.8 \mathrm{~m} / \mathrm{s} \text { and } \\
\text { Grip strength and RMM below } \\
\text { cut-off points }\end{array}$ & $\begin{array}{l}\text { 4-meter walking test } \\
\text { Maltron Bioscan 920-II with } \\
\text { Maltron calculation } \\
\text { Jamar dynamometer }\end{array}$ & $\begin{array}{l}\text { Class } 1 \\
\text { Class } 2 \\
\\
\\
\text { Class } 1 \\
\text { Class } 2\end{array}$ & $\begin{array}{l}\leq 0.8 \mathrm{~m} / \mathrm{s} \\
+ \\
37 \% \\
31 \% \\
\\
>0.8 \mathrm{~m} / \mathrm{s}+ \\
\mathrm{BMI}^{\ddagger} \leq 24 \quad \leq 29 \mathrm{~kg} \\
\mathrm{BMl} 24.1-26 \leq 30 \mathrm{~kg} \\
\mathrm{BMl} 26.1-28 \leq 30 \mathrm{~kg} \\
\mathrm{BMl}>28 \quad \leq 32 \mathrm{~kg} \\
+ \\
37 \% \\
31 \%\end{array}$ & $\begin{array}{l}\leq 0.8 \mathrm{~m} / \mathrm{s} \\
+ \\
28 \% \\
22 \% \\
\\
>0.8 \mathrm{~m} / \mathrm{s}+ \\
\mathrm{BMI} \leq 23 \quad \leq 17 \mathrm{~kg} \\
\mathrm{BMI} 23.1-26 \leq 17.3 \mathrm{~kg} \\
\mathrm{BMI} 26.1-29 \leq 18 \mathrm{~kg} \\
\mathrm{BMI}>29 \quad \leq 21 \mathrm{~kg} \\
+ \\
28 \% \\
22 \%\end{array}$ \\
\hline & D4 & $\begin{array}{l}\text { EWGSOP } \\
\text { Gait speed } \leq 0.8 \mathrm{~m} / \mathrm{s} \text { and } \\
\text { RMM below cut-off point } \\
\text { or } \\
\text { Gait speed }>0.8 \mathrm{~m} / \mathrm{s} \text { and } \\
\text { Grip strength and RMM below } \\
\text { cut-off points }\end{array}$ & $\begin{array}{l}\text { 4-meter walking test } \\
\text { Maltron Bioscan 920-II with } \\
\text { Janssen calculation } \\
\text { Jamar dynamometer }\end{array}$ & $\begin{array}{l}\text { Class } 1 \\
\text { Class } 2 \\
\\
\text { Class } 1 \\
\text { Class } 2\end{array}$ & $\begin{array}{l}\leq 0.8 \mathrm{~m} / \mathrm{s}+ \\
37 \% \\
31 \% \\
\\
>0.8 \mathrm{~m} / \mathrm{s}+ \\
\mathrm{BMl} \leq 24 \quad \leq 29 \\
\mathrm{~kg} \\
\mathrm{BMl} 24.1-26 \leq 30 \mathrm{~kg} \\
\mathrm{BMl} 26.1-28 \leq 30 \mathrm{~kg} \\
\mathrm{BMl}>28 \quad \leq 32 \\
\mathrm{~kg} \\
+ \\
37 \% \\
31 \%\end{array}$ & $\begin{array}{l}\leq 0.8 \mathrm{~m} / \mathrm{s}+ \\
28 \% \\
22 \% \\
\\
>0.8 \mathrm{~m} / \mathrm{s}+ \\
\mathrm{BMI} \leq 23 \quad \leq 17 \mathrm{~kg} \\
\mathrm{BMI} 23.1-26 \leq 17.3 \mathrm{~kg} \\
\mathrm{BMl} 26.1-29 \leq 18 \mathrm{~kg} \\
\mathrm{BMI}>29 \quad \leq 21 \mathrm{~kg} \\
+ \\
28 \% \\
22 \%\end{array}$ \\
\hline & D5 & $\begin{array}{l}\text { EWGSOP algorithm } \\
\text { Gait speed } \leq 0.8 \mathrm{~m} / \mathrm{s} \text { and } \\
\text { SMI below cut-off point } \\
\text { or } \\
\text { Gait speed }>0.8 \mathrm{~m} / \mathrm{s} \text { and } \\
\text { Grip strength and SMI below } \\
\text { cut-off points } \text { s }^{10,25}\end{array}$ & $\begin{array}{l}\text { 4-meter walking test } \\
\text { Maltron Bioscan 920-II with } \\
\text { Maltron calculation } \\
\text { Jamar dynamometer }\end{array}$ & $\begin{array}{l}\text { Moderate } \\
\text { Severe } \\
\text { Moderate } \\
\text { Severe }\end{array}$ & $\begin{array}{l}\leq 0.8 \mathrm{~m} / \mathrm{s}+ \\
10.75 \\
8.50 \mathrm{~kg} / \mathrm{m}^{2} \\
\\
>0.8 \mathrm{~m} / \mathrm{s}+ \\
<30 \mathrm{~kg}+ \\
10.75 \\
8.50 \mathrm{~kg} / \mathrm{m}^{2}\end{array}$ & $\begin{array}{l}\leq 0.8 \mathrm{~m} / \mathrm{s}+ \\
6.75 \\
5.75 \mathrm{~kg} / \mathrm{m}^{2} \\
\\
>0.8 \mathrm{~m} / \mathrm{s}+ \\
<20 \mathrm{~kg}+ \\
6.75 \\
5.75 \mathrm{~kg} / \mathrm{m}^{2}\end{array}$ \\
\hline & D6 & $\begin{array}{l}\text { EWGSOP algorithm } \\
\text { Gait speed } \leq 0.8 \mathrm{~m} / \mathrm{s} \text { and } \\
\text { SMI below cut-off point } \\
\text { or } \\
\text { Gait speed }>0.8 \mathrm{~m} / \mathrm{s} \text { and } \\
\text { Grip strength and SMI below } \\
\text { cut-off points } \mathrm{s}^{3,10,25}\end{array}$ & $\begin{array}{l}\text { 4-meter walking test } \\
\text { Maltron Bioscan 920-II with } \\
\text { Janssen calculation } \\
\text { Jamar dynamometer }\end{array}$ & $\begin{array}{l}\text { Moderate } \\
\text { Severe } \\
\text { Moderate } \\
\text { Severe }\end{array}$ & $\begin{array}{l}\leq 0.8 \cdot \mathrm{m} / \mathrm{s}+ \\
10.75 \\
8.50 \mathrm{~kg} / \mathrm{m}^{2} \\
\\
>0.8 \mathrm{~m} / \mathrm{s}+ \\
<30 \mathrm{~kg}+ \\
10.75 \\
8.50 \mathrm{~kg} / \mathrm{m}^{2}\end{array}$ & $\begin{array}{l}\leq 0.8 \mathrm{~m} / \mathrm{s}+ \\
6.75 \\
5.75 \mathrm{~kg} / \mathrm{m}^{2} \\
\\
>0.8 \mathrm{~m} / \mathrm{s}+ \\
<20 \mathrm{~kg}+ \\
6.75 \\
5.75 \mathrm{~kg} / \mathrm{m}^{2}\end{array}$ \\
\hline
\end{tabular}




\section{Sarcopenia prevalence}

Table 2 shows sarcopenia prevalence using the different diagnostic criteria. Sarcopenia prevalence differed when various diagnostic criteria were applied. According to definitions that only take muscle mass into account ( $A 1, A 2, A 3$, and $A 4)$, prevalence ranged from $26-70 \%$ for women and from $46-100 \%$ for men. When sarcopenia was defined as low grip strength (B), the prevalence was $75 \%$ for both men and women. According to definitions that include a combination of low muscle mass and low gait speed (IWGS: C1-C4 and SIG: C5-C6), sarcopenia prevalence ranged from $26-69 \%$ for women and from $42-96 \%$ for men. According to the EWGSOP diagnostic criteria (D1-D6) with different cut-off points for low grip strength and low RMM or SMI for muscle mass, sarcopenia prevalence ranged from $26-70 \%$ for women and from $42-92 \%$ for men. The prevalence of sarcopenia according to the EWGSOP algorithm used with the Maltron and Janssen calculations (D5-D6) was $67 \%$ and $26 \%$ for women and $92 \%$ and $71 \%$ for men, respectively. Figure 2 shows the influence of both the Jansen and Maltron calculations for estimating muscle mass on the prevalence of sarcopenia.
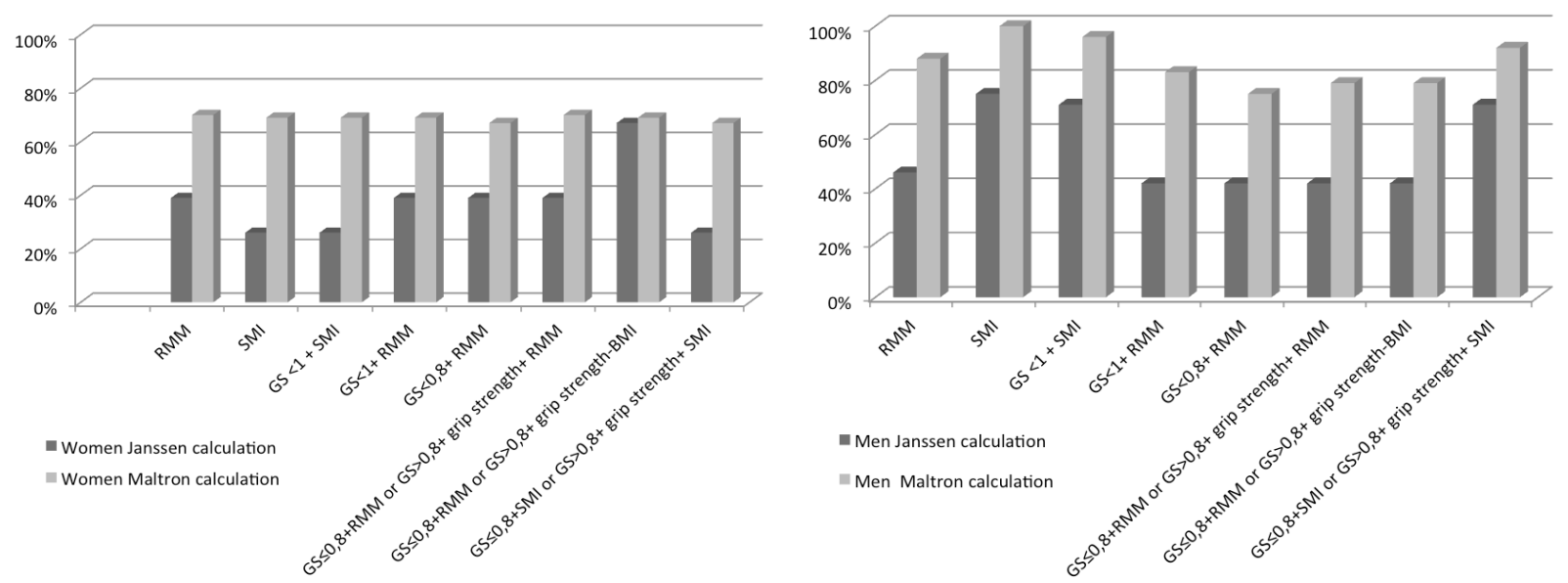

Figure 2: Prevalence of sarcopenia in hospitalized geriatric patients $(n=85)$ according to different criteria with Janssen and Maltron calculations (Left: women $(n=61) ;$ Right: men $(n=24)$ )

The prevalence of sarcopenia was higher when the Maltron calculation was applied. Twelve (14\%) geriatric patients ( 5 men and 7 women) were sarcopenic according to all 17 definitions. When only the Janssen calculation was applied for measuring muscle mass, 15 (18\%) patients were sarcopenic according to all definitions; when only the Maltron calculation was applied, 30 $(35 \%)$ were sarcopenic. 
Table 2: Prevalence of sarcopenia in study population $(n=85)$, based on different criteria: skeletal muscle mass, EWGSOP, IWGS and SIG.

\begin{tabular}{|c|c|c|c|c|c|}
\hline Criterion & Code ${ }^{*}$ & Formula & $\begin{array}{l}\text { Cut-off point, } \\
\text { sarcopenia } \\
\text { present }\end{array}$ & $\begin{array}{l}\text { Men } \\
n=24\end{array}$ & $\begin{array}{l}\text { Women } \\
n=61\end{array}$ \\
\hline \multirow[t]{4}{*}{ Muscle mass } & $\mathrm{A} 1$ & RMM & $\begin{array}{l}\text { Class I } \\
\text { Class II } \\
\text { Total }\end{array}$ & $\begin{array}{r}16(67 \%) \\
5(21 \%) \\
21(88 \%)\end{array}$ & $\begin{array}{r}36(59 \%) \\
7(11 \%) \\
43(70 \%)\end{array}$ \\
\hline & A2 & RMM & $\begin{array}{l}\text { Class I } \\
\text { Class II } \\
\text { Total }\end{array}$ & $\begin{array}{r}9(38 \%) \\
2(8 \%) \\
11(46 \%)\end{array}$ & $\begin{array}{r}23(37 \%) \\
1(2 \%) \\
24(39 \%)\end{array}$ \\
\hline & A3 & SMI & $\begin{array}{l}\text { Moderate } \\
\text { Severe } \\
\text { Total }\end{array}$ & $\begin{array}{l}12(50 \%) \\
12(50 \%) \\
24(100 \%)\end{array}$ & $\begin{array}{r}34(56 \%) \\
8(13 \%) \\
42(69 \%)\end{array}$ \\
\hline & A4 & SMI & $\begin{array}{l}\text { Moderate } \\
\text { Severe } \\
\text { Total }\end{array}$ & $\begin{array}{r}13(54 \%) \\
5(21 \%) \\
18(75 \%)\end{array}$ & $\begin{array}{r}10(16 \%) \\
6(10 \%) \\
16(26 \%)\end{array}$ \\
\hline Muscle strength & $\mathrm{B}$ & Grip strength & Sarcopenia & $18(75 \%)$ & $46(75 \%)$ \\
\hline \multirow[t]{6}{*}{$\begin{array}{l}\text { Muscle mass } \\
\text { and } \\
\text { gait speed }\end{array}$} & C1 & $\begin{array}{l}\text { Gait speed }<1 \mathrm{~m} / \mathrm{s} \text { and } \\
\text { SMI }\end{array}$ & $\begin{array}{l}\text { Moderate } \\
\text { Severe } \\
\text { Total }\end{array}$ & $\begin{array}{l}12(50 \%) \\
11(46 \%) \\
23(96 \%)\end{array}$ & $\begin{array}{r}34(56 \%) \\
8(13 \%) \\
42(69 \%)\end{array}$ \\
\hline & $\mathrm{C} 2$ & $\begin{array}{l}\text { Gait speed }<1 \mathrm{~m} / \mathrm{s} \text { and } \\
\text { SMI }\end{array}$ & $\begin{array}{l}\text { Moderate } \\
\text { Severe } \\
\text { Total }\end{array}$ & $\begin{array}{r}13(54 \%) \\
4(21 \%) \\
17(75 \%) \\
\end{array}$ & $\begin{array}{r}10(16 \%) \\
6(10 \%) \\
16(26 \%) \\
\end{array}$ \\
\hline & C3 & $\begin{array}{l}\text { Gait speed }<1 \mathrm{~m} / \mathrm{s} \text { and } \\
\text { RMM }\end{array}$ & $\begin{array}{l}\text { Class } 1 \\
\text { Class } 2 \\
\text { Total }\end{array}$ & $\begin{array}{r}16(67 \%) \\
4(17 \%) \\
20(83 \%)\end{array}$ & $\begin{array}{r}36(59 \%) \\
6(10 \%) \\
42(69 \%)\end{array}$ \\
\hline & $\mathrm{C} 4$ & $\begin{array}{l}\text { Gait speed }<1 \mathrm{~m} / \mathrm{s} \text { and } \\
\text { RMM below cut-off point }\end{array}$ & $\begin{array}{l}\text { Class } 1 \\
\text { Class } 2 \\
\text { Total } \\
\end{array}$ & $\begin{array}{r}8(33 \%) \\
2(8 \%) \\
10(42 \%) \\
\end{array}$ & $\begin{array}{r}23(38 \%) \\
1(2 \%) \\
24(39 \%) \\
\end{array}$ \\
\hline & C5 & $\begin{array}{l}\text { Gait speed }<0.8 \mathrm{~m} / \mathrm{s} \text { and } \\
\text { RMM }\end{array}$ & $\begin{array}{l}\text { Class } 1 \\
\text { Class } 2 \\
\text { Total } \\
\end{array}$ & $\begin{array}{r}14(58 \%) \\
4(17 \%) \\
18(75 \%) \\
\end{array}$ & $\begin{array}{r}5(57 \%) \\
6(10 \%) \\
41(67 \%) \\
\end{array}$ \\
\hline & $\mathrm{C} 6$ & $\begin{array}{l}\text { Gait speed }<0.8 \mathrm{~m} / \mathrm{s} \text { and } \\
\text { RMM }\end{array}$ & $\begin{array}{l}\text { Class } 1 \\
\text { Class } 2 \\
\text { Total } \\
\end{array}$ & $\begin{array}{r}8(34 \%) \\
2(8 \%) \\
10(42 \%) \\
\end{array}$ & $\begin{array}{r}23(37 \%) \\
1(2 \%) \\
24(39 \%) \\
\end{array}$ \\
\hline \multirow[t]{6}{*}{$\begin{array}{l}\text { Muscle mass } \\
\text { and gait speed } \\
\text { and } \\
\text { grip strength }\end{array}$} & D1 & $\begin{array}{l}\text { Gait speed } \leq 0.8 \mathrm{~m} / \mathrm{s} \text { and } \\
\text { RMM } \\
\text { or } \\
\text { Gait speed }>0.8 \mathrm{~m} / \mathrm{s} \text { and } \\
\text { Grip strength and RMM }\end{array}$ & $\begin{array}{l}\text { Class } 1 \\
\text { Class } 2 \\
\text { Class } 1 \\
\text { Class } 2 \\
\text { Total }\end{array}$ & $\begin{array}{r}14(58 \%) \\
4(17 \%) \\
1(4 \%) \\
0(0 \%) \\
19(79 \%)\end{array}$ & $\begin{array}{r}35(57 \%) \\
6(10 \%) \\
1(2 \%) \\
1(2 \%) \\
43(70 \%)\end{array}$ \\
\hline & D2 & $\begin{array}{l}\text { Gait speed } \leq 0.8 \mathrm{~m} / \mathrm{s} \text { and } \\
\text { RMM } \\
\text { or } \\
\text { Gait speed }>0.8 \mathrm{~m} / \mathrm{s} \text { and } \\
\text { Grip strength and RMM }\end{array}$ & $\begin{array}{l}\text { Class } 1 \\
\text { Class } 2 \\
\text { Class } 1 \\
\text { Class } 2 \\
\text { Total }\end{array}$ & $\begin{array}{r}8(34 \%) \\
2(8 \%) \\
0(0 \%) \\
0(0 \%) \\
10(42 \%)\end{array}$ & $\begin{array}{r}23(37 \%) \\
1(2 \%) \\
0(0 \%) \\
0(0 \%) \\
24(39 \%) \\
\end{array}$ \\
\hline & D3 & $\begin{array}{l}\text { Gait speed } \leq 0.8 \mathrm{~m} / \mathrm{s} \text { and } \\
\text { RMM } \\
\text { or } \\
\text { Gait speed }>0.8 \mathrm{~m} / \mathrm{s} \text { and } \\
\text { Grip strength and RMM }\end{array}$ & $\begin{array}{l}\text { Class } 1 \\
\text { Class } 2 \\
\text { Class } 1 \\
\text { Class } 2 \\
\text { Total }\end{array}$ & $\begin{array}{r}14(58 \%) \\
4(17 \%) \\
1(4 \%) \\
0(0 \%) \\
19(79 \%)\end{array}$ & $\begin{array}{r}35(57 \%) \\
6(10 \%) \\
1(2 \%) \\
0(0 \%) \\
42(69 \%)\end{array}$ \\
\hline & D4 & $\begin{array}{l}\text { Gait speed } \leq 0.8 \mathrm{~m} / \mathrm{s} \text { and } \\
\text { RMM } \\
\text { or } \\
\text { Gait speed }>0.8 \mathrm{~m} / \mathrm{s} \text { and } \\
\text { Grip strength and RMM }\end{array}$ & $\begin{array}{l}\text { Class } 1 \\
\text { Class } 2 \\
\text { Class } 1 \\
\text { Class } 2 \\
\text { Total } \\
\end{array}$ & $\begin{array}{r}8(34 \%) \\
2(8 \%) \\
0(0 \%) \\
0(0 \%) \\
10(42 \%) \\
\end{array}$ & $\begin{array}{r}35(57 \%) \\
6(10 \%) \\
0(0 \%) \\
0(0 \%) \\
41(67 \%) \\
\end{array}$ \\
\hline & D5 & $\begin{array}{l}\text { Gait speed } \leq 0.8 \mathrm{~m} / \mathrm{s} \text { and } \\
\text { SMl below cut-off point } \\
\text { or } \\
\text { Gait speed }>0.8 \mathrm{~m} / \mathrm{s} \text { and } \\
\text { Grip strength and } \\
\text { SMl below cut-off points }\end{array}$ & $\begin{array}{l}\text { Moderate } \\
\text { Severe } \\
\text { Moderate } \\
\text { Severe } \\
\text { Total }\end{array}$ & $\begin{array}{r}11(46 \%) \\
10(42 \%) \\
0(0 \%) \\
1(4 \%) \\
22(92 \%) \\
\end{array}$ & $\begin{array}{r}32(52 \%) \\
7(11 \%) \\
2(3 \%) \\
0(0 \%) \\
41(67 \%) \\
\end{array}$ \\
\hline & D6 & $\begin{array}{l}\text { Gait speed } \leq 0.8 \mathrm{~m} / \mathrm{s} \text { and } \\
\text { SMI below cut-off point } \\
\text { or } \\
\text { Gait speed }>0.8 \mathrm{~m} / \mathrm{s} \text { and } \\
\text { Grip strength and } \\
\text { SMI below cut-off points }\end{array}$ & $\begin{array}{l}\text { Moderate } \\
\text { Severe } \\
\text { Moderate } \\
\text { Severe } \\
\text { Total }\end{array}$ & $\begin{aligned} & 12(50 \%) \\
& 4(17 \%) \\
& 1(4 \%) \\
& 0(0 \%) \\
& 17(71 \%)\end{aligned}$ & $\begin{array}{r}10(16 \%) \\
6(10 \%) \\
0(0 \%) \\
0(0 \%) \\
16(26 \%)\end{array}$ \\
\hline
\end{tabular}

* Code as defined in Table 1 . 


\section{Discussion}

We found a huge variation in sarcopenia prevalence when applying different criteria. This variation is consistent with results from the Leiden Longevity Study, in which sarcopenia prevalence also varied when different criteria were used. In the Leiden community cohort, who were aged 70 years and older, the prevalence varied from $0-45.2 \%$ for men and $0-25.8 \%$ for women (28).

Analysis of 119 acutely ill older patients (mean age of 80.4 years and $34.4 \%$ female) who were admitted to a geriatric unit in an academic medical department showed a sarcopenia prevalence of $26 \%$. In this study the EWGSOP algorithm with assessment of muscle mass by bio-impedance analysis, muscle strength by handheld dynamometer and gait speed by the four-meter walking test was used (15). Other researchers found a similar prevalence: $25.3 \%$ in 198 patients (mean age of 82.8 years and $70.2 \%$ female) from an acute care geriatric ward of a general hospital. They used the cut-off points of the EWGSOP algorithm, although they used the Short Physical Performance Battery instead of the fourmeter walking test (16). A similar sarcopenia prevalence (28\%) was found in 770 acutely ill older patients (mean age of 81.7 years and $56 \%$ female) who were admitted to geriatric and internal medicine acute care wards at seven Italian hospitals (17). In that study the EWGSOP algorithm, with assessment of muscle mass by bio-impedance analysis (including the Janssen calculation), grip strength and gait speed (four-meter walking test) was used.

The higher sarcopenia prevalence we found compared to the above findings might be explained because our geriatric patients had a higher average age ( 84 years, $S D=5.8$ ) and were more physically frail (mean Fried score of 4). The higher prevalence of sarcopenia in our study might also be explained because of the impact of relatively acute loss of muscle mass due to acute illness, as shown in earlier research (5).

Another possible explanation for the big variance in sarcopenia prevalence might be the impact of the different calculations used in the BIA estimation of muscle mass as shown in Figure 2. Overall, the rate of sarcopenia prevalence assessed by applying definitions that use the Janssen calculation is lower than the rate assessed by applying definitions using the Maltron calculation. It is possible that the Janssen calculation might be overrating skeletal muscle mass, since it draws a direct relationship between resistance and skeletal muscle mass (13). In contrast, the Maltron calculation uses other frequencies, which allow it to calculate intracellular and extracellular water and body mass. Many hospitalized frail elderly people have hydration problems that have a direct influence on the estimation of muscle mass, especially when using the Janssen calculation. As Figure 2 shows, there is little variance in sarcopenia prevalence (67\% versus $70 \%$ ) for women when using the Maltron calculation and different definitions. As far as we know, this is the second study to use BIA with the Maltron 
calculation. A high correlation between BIA with Maltron calculation and MRI was shown in an aged community population (25). The higher prevalence of sarcopenia in men compared to women in all criteria (with the exception of criterion with code D4; Table 1) is consistent with results from earlier studies (27).

In our study, few geriatric patients $(12 ; 14 \%)$ were sarcopenic according to all 17 different criteria (as shown in Table 1). This finding is consistent with the results of the Leiden Longevity Study, in which only 1 of 130 participants $(0.2 \%)$ was identified as sarcopenic according to seven different diagnostic criteria (28). It is relevant to know which of the criteria or components of sarcopenia are related with adverse outcomes in acutely hospitalized frail elderly people, such as those in this study's target group. McLean and colleagues found among 1869 women (mean age of 76 years) and 4411 men (mean age of 74 years), pooled from 6 cohort studies from the Foundation for the National Institutes of Health (FNIH) Sarcopenia Project, that low grip strength $(<26 \mathrm{~kg}$ men and $<16 \mathrm{~kg}$ women) and low appendicular lean mass to Body Mass Index ratio ( $<0.789$ men and $<0.512$ women) were associated with increased likelihood for incident mobility impairment $(29,30,31)$. However validation of these candidate criteria for clinically relevant weakness and low lean mass (measured with BIA instead of dual-energy x-ray absorptiometry) in acutely hospitalized frail elderly people is needed.

Our study had some limitations. First, it was a single center study, limited to one acute care geriatric ward of a Dutch general hospital and only included geriatric patients who were mobile prior to hospitalization. Second, participants were only recruited between March and June, so it is possible that seasonal variations of disease manifestations might have affected the study findings. The influence of season has been previously demonstrated for variation in disease, vitamin D level, physical activity, ankle dorsiflexion strength and hours spent outside (32). A third limitation of this study is the fact that we were missing values for $43(33 \%)$ of the eligible patients. Although they had similar ages and were equally physically frail (data not shown) compared to the included patients, we cannot exclude possible influences their inclusion may have had on our findings. Finally, we were not able to differentiate between sarcopenia and cachexia in this study. These two conditions may overlap in acutely ill geriatric patients because they share common etiological pathways. Therefore we have to be cautious with generalizing our prevalence results.

\section{Conclusion}

Sarcopenia is highly prevalent in an acute hospitalized geriatric population, although the prevalence varies widely depending on the diagnostic criteria applied. In this study, almost all the geriatric patients (96\%) had a low gait speed. Furthermore, using the Maltron calculation to estimate skeletal muscle mass found a higher prevalence of sarcopenia and a higher rate of 
sarcopenic patients according to all definitions than did the Janssen calculation. Sarcopenia prevalence was almost $70 \%$ for female geriatric patients, according to all definitions including muscle mass. The prevalence varied more widely for men when the SMI, RMM, SIG, IWGS and EWGSOP criteria were applied.

We clearly found that current definitions of sarcopenia are identifying different patients. In accordance with the findings from McLean and colleagues it might be possible to diagnose sarcopenia in acutely ill geriatric patients based only on grip strength and a muscle mass index, because almost all the patients in our study sample had low gait speed (29). Since acute conditions are often related to momentary functional impairments, gait speed may be less suitable as a criterion for diagnosing sarcopenia in acutely ill geriatric patients. From a clinical point of view and in accordance with the findings from the FNIH Sarcopenia Project, it is important to select sarcopenic geriatric patients with adverse outcomes like loss of mobility or functional decline, which can be prevented with adequate treatment $(29,30,31)$. A prospective study is needed to discover which specific criteria of sarcopenia, for example the new candidate criteria from the FNIH Sarcopenia Project, can predict these adverse outcomes. This may be the starting point for treating sarcopenic geriatric patients and preventing unnecessary and undesired functional loss. 


\section{References}

1. Covinsky K, Pierluissi E, Johnston C. Hospitalization Associated Disability. JAMA 2011;306:1782-1793

2. Merino Martín S, Cruz Jentoft AJ. Impact of hospital admission on functional and cognitive measures in older subjects. Eur Geriatr Med 2012 Aug;3: 208-212.

3. Janssen I, Heymsfield SB, Ross R. Low relative skeletal muscle mass (sarcopenia) in older persons is associated with functional impairment and physical disability. J Am Geriatr Soc 2002; 50:889-96.

4. Gale CR, Martyn CN, Cooper C, Sayer AA (2007), Grip strength, body composition, and mortality. Int J Epidemiol 36: 228-235

5. Kortebein et al. Effect of 10 Days of Bed Rest on Skeletal Muscle in Healthy Older Adults JAMA 2007; 297:16;1772- 1774

6. Ling $\mathrm{CHY}$ et al. Accuracy of direct segmental multi-frequency bio impedance analysis in the assessment of total body and segmental body composition in middle aged adult population. Clinical nutrition 30(2011) 610-615

7. Janssen I, Baumgartner R, Ross $\mathrm{R}$ et al. Skeletal muscle cut points associated with elevated physical disability risk in older men and women. Am J Epidemiol 2004; 159: 413-21.

8. Cesari M, Kritchevsky SB, Newman $A B$ et al. Added value of physical performance measures in predicting adverse health-related events: results from the health, aging and body composition study. J Am Geriatr Soc 2009; 57: 251-9.

9. Rosenberg I. Summary comments: epidemiological and methodological problems in determining nutritional status of older persons. Am J Clin Nutr 1989;50:1231-3

10. Cruz-Jentoft AJ, Baeyens JP, Bauer JM, et al. Sarcopenia: European consensus on definition and diagnosis: Report of the European Working Group on Sarcopenia in Older People. Age Ageing 2010; 39: 412-423.

11. Fielding RA, Vellas B, Evans WJ, et al. Sarcopenia: an undiagnosed condition in older adults. Current consensus definition: prevalence, etiology, and consequences. International Working Group on Sarcopenia. J Am Med Dir Assoc 2011; 12: 249-256.

12. Morley JE, Abbatecola AM, Argiles JM, et al. Sarcopenia with limited mobility: an international consensus. J Am Med Dir Assoc 2011; 12: 403-409.

13. Janssen I, Heymsfield SB, Baumgartner RN, Ross R. Estimation of skeletal muscle mass by bioelectrical impedance analysis. J Appl Physiol 2000 Aug;89:465-71.

14. Kyle UG, Genton L, Hans D, Pichard C. Validation of a bioelectrical impedance analysis equation to predict appendicular skeletal muscle mass (ASMM). Clin Nutr 2003 Dec;22:537-43.

15. Rossi AP, Fantin F, Micciolo R, et al. Identifying sarcopenia in acute care setting patients. J Am Med Dir Assoc 2014; 15: 303.e7-303.e12.

16. Smoliner C, Sieber CC, Wirth R. Prevalence of sarcopenia in geriatric hospitalized patients. J Am Med Dir Assoc 2014; 15: 267-272.

17. Vetrano DL, Landi F, Volpato S, et al. Association of Sarcopenia With Short- and Longterm Mortality in Older Adults Admitted to Acute Care Wards: Results From the CRIME Study. J Gerontol A Biol Sci Med Sci (2014) doi: 10.1093/gerona/glu034.

18. Fried LP, Tangen CM, Walston $\mathrm{J}$, et al. Frailty in older adults: evidence for a phenotype. J Gerontol A Biol Sci Med Sci 2001 Mar;56:M146-56.

19. Malnutrition Advisory Group, British Association of Parenteral and Enteral Nutrition. October 2008. Malnutrition Universal Screening Tool. The Malnutrition Universal Screening Tool (MUST) is reproduced here with the kind permission of BAPEN.

20. Nagaratnam N, Gayagay G. Validation of the Cumulative Illness Rating Scale (CIRS) in hospitalized nonagenarians. Arch Gerontol Geriatr 2007 Jan-Feb;44:29-36.

21. Kruizenga HM, Seidell JC, Vet $\mathrm{H}$ de, et al. Development and validation of a hospital screening tool for malnutrition: the short nutritional assessment questionnaire (SNAQ).Clin Nutr 2005; 24, 75-82. 
22. Katz S, Ford AB, Moskowitz RW, Jackson BA, Jaffe MW. Studies of illness in the aged. The index of ADL: a standardized measure of biological and psychosocial function. JAMA 1963 Sep 21;185:914-919.

23. Roberts $\mathrm{H}$ et al. A review of the measurement of grip strength in clinical and epidemiological studies: towards a standardized approach. Age and Ageing 2011; 40:423-429

24. Oldfields OD. The assessment and analysis of handedness: The Edinburgh Inventory. Neuropsychology 1971; 9: 97-113

25. Chien MY, Ta-Yi Huang, Ying-Tai Wu. Prevalence of sarcopenia estimated using a bioelectric impedance analysis. Prediction equation in community-dwelling elderly people in Taiwan. J Am Geriatr Soc 2008; 56:1710-5.

26. Graham JE, Ostir GV, Fisher SR, Ottenbacher KJ. Assessing walking speed in clinical research: A systematic review. Journal of Evaluation in Clinical Practice 2008;14,552562.

27. Thomas D. Sarcopenia. Clin Geriatr Med $26 \quad$ (2010) 331-346 doi:10.1016/j.cger.2010.02.012

28. Bijlsma AY, Meskers CGM, Ling CHY et al. Defining sarcopenia: the impact of different diagnostic criteria on the prevalence of sarcopenia in a large middle aged cohort. Age (Dordr.) Epub 2012 Febr 8

29. McLean RR, Shardell MD, Alley DE et al. Criteria for Clinically Relevant Weakness and Low Lean Mass and Their Longitudinal Association With Incident Mobility Impairment and Mortality: The Foundation for the National Institutes of Health (FNIH) Sarcopenia Project. J Gerontol A Biol Sci Med Sci 2014 May;69:576-583

30. Studenski SA, Peters KW, Alley DE et al. The FNIH Sarcopenia Project: Rationale, Study Description, Conference Recommendations, and Final Estimates. J Gerontol A Biol Sci Med Sci. 2014 May; 69: 547-558

31. Dam TT, Peters KW, Fragala M et al. An Evidence-Based Comparison of Operational Criteria for the Presence of Sarcopenia. J Gerontol A Biol Sci Med Sci. 2014 May; 69: 584-590.

32. Bird ML, Hill KD, Robertson IK, Ball MJ, Pittaway J, Williams AD. Serum [25(OH)D] status, ankle strength and activity show seasonal variation in older adults: relevance for winter falls in higher latitudes. Age Ageing 2013 Mar;42:181-5. 


\section{CHAPTER 3}

\section{Sarcopenia is related to mortality in the}

\section{acutely hospitalized geriatric patient}

Keywords: gait speed, skeletal muscle mass, fat mass, phase angle, sarcopenia

Walther MWH Sipers, Wout de Blois, Jos MGA Schols,

Luc JC van Loon and Lex B Verdijk

Journal of Nutrition, Health \& Aging. 2019;23(2):128-137

doi: 10.1007/s12603-018-1134-1 


\section{Abstract}

Background: Sarcopenia is defined as low skeletal muscle mass with poor physical performance, representing a strong prognostic factor for mortality in older people. Although highly prevalent in hospitalized geriatric patients, it is unknown whether sarcopenia can also predict mortality in these patients.

Objective: To determine the association between sarcopenia according the criteria of the European Working Group on Sarcopenia in Older People (EWGSOP), International Working Group on Sarcopenia (IWGS), Special Interest Group of Sarcopenia, Cachexia and Wasting Disorders (SIG) and Foundation for the National Institutes of Health (FNIH) and 2-year mortality in acutely hospitalized geriatric patients.

Design: 81 patients $(84 \pm 5 \mathrm{y})$ admitted to the acute geriatric ward participated in this study. Body composition assessment (bio-impedance, Maltron Bioscan 920-II) and physical performance tests were performed, and mortality information was retrieved through patient files.

Results: Prevalence rates of sarcopenia were 51\% (EWGSOP), 75\% (IWGS), 69\% (SIG), and $27 \%$ (FNIH). Based on Cox proportional hazard ratio (HR) analysis, 2-year mortality was significantly higher in sarcopenic patients versus non-sarcopenic patients when using the EWGSOP (2-y: HR 4.310; Cl-95\%:2.092-8.850; P<0.001) and FNIH criteria (2-y: HR 3.571; $\mathrm{Cl-95 \% :1.901-6.711;P<0.001).} \mathrm{Skeletal} \mathrm{muscle} \mathrm{mass} \mathrm{index,} \mathrm{fat} \mathrm{mass} \mathrm{index,} \mathrm{body} \mathrm{mass} \mathrm{index,}$ phase angle and gait speed were significantly lower in the geriatric patients who deceased after 2 years versus those who were still alive. Cox proportional HR analyses showed that higher phase angle (HR 0.678; $\mathrm{Cl}-95 \%: 0.531-0.864 ; P=0.002)$ and higher fat mass index (HR 0.839; Cl-95\%:0.758-0.928; $P=0.001)$ significantly reduced $2-y$ mortality probability. Combining sarcopenia criteria and separate patient characteristics finally resulted in a model in which HRs for sarcopenia (EWGSOP and FNIH) as well as phase angle significantly predicted mortality probability.

Conclusion: Sarcopenia is prevalent in acutely hospitalized geriatric patients and is associated with significantly higher 2-year mortality according the EWGSOP and FNIH criteria. 


\section{Introduction}

Aging is associated with progressive loss of skeletal muscle mass and strength, commonly termed sarcopenia $(1,2)$. This age-related decline in skeletal muscle mass and strength impairs functional performance, leading to a decreased level of independence and an increased mortality (3-5). Prevalence of sarcopenia varies widely and depends on the definition, the population studied, and the methodology used for measuring different domains of sarcopenia such as muscle mass, gait speed and grip strength. Hence, sarcopenia prevalence in community dwelling older people has been estimated at $5.3 \%$ for men and $13.3 \%$ for women according to the European Working Group on Sarcopenia in Older People (EWGSOP) definition; $5.1 \%$ for men and $11.8 \%$ for women according to the International Working Group on Sarcopenia (IWGS) definition; and $1.3 \%$ for men and $2.3 \%$ for women according to the Foundation for the National Institutes of Health $(\mathrm{FNIH})$ criteria $(6,7)$. Furthermore, prevalence tends to be higher in acute care hospital settings $(1,8)$ with the highest prevalence in hospitalized geriatric patients, ranging between $21-46 \%(9-13)$.

Handgrip strength, gait speed and skeletal muscle mass are key features in the operational definition of the EWGSOP and FNIH, however they differ in cut off values and techniques used to assess muscle mass. The IWGS and Special Interest Group of Sarcopenia, Cachexia and Wasting Disorders (SIG) criteria of sarcopenia only incorporate gait speed and muscle mass as key features, although again with different cut off values. While there is strong agreement between the different criteria for the "no sarcopenia" situation, the percent agreement for the classification "sarcopenia" appears rather low, ranging from 5 to $32 \%$ (14). Nonetheless, sarcopenia in older people in the community is associated with increased risk of incident disability, institutionalization, and mortality; independent of whether it is defined by the EWGSOP (1), IWGS (15), or FNIH criteria $(4,6,16)$. Despite its strong prognostic value for mortality in community-dwelling elderly, and the high prevalence of sarcopenia in hospitalized geriatric patients, it is unclear to what extent sarcopenia is also associated with mortality in the hospitalized geriatric patient. The study of Cerri and colleagues (12) and Perez-Zepeda and co-workers (13) are currently the only two studies concerning mortality in sarcopenic geriatric patients admitted to respectively an acute geriatric ward and a Geriatric Management and Evaluation Unit. In their work, sarcopenia was diagnosed in $21.4 \%$ of 103 (12) and $40.1 \%$ of 172 (13) geriatric patients using the EWGSOP definition. In both studies more patients had deceased in the sarcopenic versus non-sarcopenic group following 3 (12) and 12 months of follow-up $(12,13)$, suggesting that sarcopenia in acutely ill geriatric patients may indeed be associated with increased mortality. Knowledge about mortality risk could be of value in targeting medical treatment in relation to hospitalized geriatric patients with limited life expectancy. However, there are no data concerning mortality in geriatric patients assessed 
over a more prolonged period (i.e., beyond 1 y) following hospital admission. Moreover, it is unclear whether the different sarcopenia definitions affect the relation with mortality, and/or which characteristics of sarcopenia may best explain the proposed association with mortality.

Therefore the present study evaluates whether sarcopenia according to the criteria of the EWGSOP, IWGS, SIG and FNIH is associated with mortality in acutely hospitalized geriatric patients. Secondly, we determined which hallmarks of sarcopenia and/or other patient characteristics can best predict mortality in geriatric patients admitted to the acute geriatric ward.

\section{Methods}

\section{Study sample}

Geriatric patients admitted to the acute geriatric ward of a Dutch general hospital were asked to participate in the study. The inclusion criteria were: age above $70 \mathrm{y}$, the ability to walk prior the onset of the acute illness leading to hospital admission, being frail according to the Groningen Frailty Indicator (GFI) (17) and if there was a written informed consent obtained from the patient or proxy. Patients were excluded if they had a pacemaker or an implantable cardioverter defibrillator (ICD) because of bio impedance measurement, were not able to follow instructions because of a severe delirium or dementia, or had a terminal condition. Further details of patient selection, in- and exclusion criteria and patient characteristics are described in our earlier publication (9). Measurement of gait speed, handgrip strength and body composition was done within four days after hospitalization. All patients were informed on the nature of the measurements before written informed consent was obtained from the patient or proxy. This study complied with the guidelines set out in the Declaration of Helsinki and was approved by the Ethics Committee of Sittard-Heerlen, the Netherlands (number 13-N60). From 128 eligible hospitalized geriatric patients, 47 patients were excluded $(n=38$ incomplete data for sarcopenia assessment, $n=9$ technical problems with bio-impedance or handgrip assessment), leaving a total of 81 patients included in this study.

Relevant patient characteristics were retrieved from the medical and nursing files. These included sex, age, living situation, diagnosed medical conditions, medical history and activities of daily living prior to the acute illness that led to hospital admission. Frailty was assessed using the GFI and Fried criteria. The GFI assesses the loss of functions and resources using 15 items divided over the physical, cognitive, social, and psychological domain. A score of 1 for an item indicates a problem and a total score of 4 or higher indicates frailty (17). The Fried criteria assess physical frailty based on 5 items: unintentional weight loss, weakness, self- 
reported exhaustion, slow walking speed, and low physical activity; a score of 3 or higher indicates physical frailty (18). Height was estimated to the nearest $\mathrm{cm}$ by measuring ulna length because many patients were temporarily bedridden (19). Bodyweight was measured to the nearest $0.1 \mathrm{~kg}$ on a sitting weight scale (SECA, Model 959). Several standard medical questionnaires and valid scales like, cumulative illness rating scale (CIRS), Short Nutritional Assessment Questionnaire (SNAQ), Katz ADL-6 and Mini Mental State Examination (MMSE) were included because of possible associations with mortality. For the 81 patients included, GFI data were missing in 2 patients, SNAQ data were missing in 2 patients, Katz-ADL data were missing in 2 patients and MMSE data were missing in 12 patients.

\section{Body composition measurement}

The Maltron BioScan 920-II, a multi-frequency multi-segmental bio-impedance (mf-ms BIA) device, was used to measure skeletal muscle mass (SMM), fat mass (FM), body cell mass (BCM) and phase angle (PA). The Maltron Bioscan 920-II has been validated for the assessment of whole body composition and segmental lean mass in elderly people (20). Phase angle has been suggested as a variable of interest from bioelectrical impedance analysis given that this variable is independent of body height and weight. It is calculated from the directly measured resistance and reactance and is associated with membrane structure and function and it is an indicator of tissue hydration and nutritional status (21). The Maltron BioScan 920-II has an eight-point electrode system, which separately measures impedance of the patient's trunk, arms and legs at four different frequencies $(5 \mathrm{kHz}, 50 \mathrm{kHz} 100 \mathrm{~Hz}$ and 200 $\mathrm{Hz}$ ) for each body segment. Absolute skeletal muscle mass (SMM) is calculated according to the device-specific calculation called the Maltron calculation (22). Patients were measured early in the morning before breakfast, wearing only the pyjamas, as described in the user's manual. Absolute skeletal muscle mass $(\mathrm{kg})$ was converted to skeletal muscle index (SMI)

standardizing by meters squared $\left(\mathrm{kg} / \mathrm{m}^{2}\right)$. Likewise, relative muscle mass (RMM, \%) was calculated by dividing SMM by body weight) (1). Additionally, fat mass index (FMI: FM/height ${ }^{2}$ ) was calculated.

\section{Physical performance tests}

The Jamar dynamometer (Sammons Preston, Inc., Bolingbrook, IL, USA) was introduced by Bechtol (23) and is a frequently used and validated device for assessing handgrip strength in healthy elderly people. Calibration of the Jamar dynamometer was performed before, during and after cessation of the study according to the guidelines set by the manufacturer. Handgrip strength was assessed with the second handle position of the Jamar dynamometer. We applied the Southampton protocol (24). Three grip strength measurements were performed for the dominant hand, with a rest period of $30 \mathrm{~s}$ between successive attempts. All measurements 
were performed between 11 am and $2 \mathrm{pm}$. Hand dominance was estimated with Edinburgh handedness inventory (25). Maximal handgrip strength was used as marker of muscle strength in the geriatric patients.

The Short Physical Performance Battery (SPPB) and the Hierarchical Balance and Mobility (HABAM) were used to assess mobility. The SPPB consists of 3 parts: balance, gait speed and chair stand test, each scored with a maximum of 4 points. Hence, the total range is from 0 to 12 points (26) with the highest score representing the best performance. Volpato et al. showed that the SPPB has predictive value for functional decline and mortality in hospitalized elderly (27). The HABAM was developed in the 1990's, by MacKnight and Rockwood, and provides information about balance, transfers and mobility. The higher the score the better the mobility level, with scores ranging from 0-67 (28). Hubbard et al. showed that the HABAM provides useful information about disease progression in hospitalized elderly (29). Gait speed was assessed according the 4-meter walking test. The four-meter walking test has been validated in elderly people. The faster of two trials was used and the test was started from a standing still position. Patients were instructed to walk at an easy usual speed and were allowed to use a walking aid if necessary (9). A total of 20 patients were unable to walk at hospital admission due to the acute illness. In these patients, gait speed was assessed after 1 week. Classification for 'sarcopenia' vs 'no sarcopenia' (see below) was not affected by the initial lack of gait speed data (i.e., all 20 patients walked slower than $0.8 \mathrm{~m} / \mathrm{s}$ ).

\section{Criteria for sarcopenia}

Table 1 shows the different diagnostic criteria we applied, with cut-off values for sarcopenia according to four consensus groups: EWGSOP, IWGS, SIG and FNIH. The FNIH uses appendicular skeletal muscle (aSM) mass as a criterion. However aSM mass data were only available for a limited number of geriatric patients. To our knowledge there are no specific publications concerning the validation of the use of total skeletal muscle mass to replace appendicular skeletal muscle mass. However, it is known that $73-75 \%$ of total skeletal muscle mass consist of appendicular skeletal muscle mass (30). Therefore we alternatively applied the BIA criteria for low SMI based on 2 SD below mean of young adults $(1,31)$, since these data were available for the majority of our population. Likewise, muscle mass criteria for IWGS were originally based on DXA criteria, but instead we applied the BIA cut off values for SMI according the NHANES III muscle mass criteria (1). All criteria used to determine whether a patient was sarcopenic or non-sarcopenic were based on measurements of muscle mass by BIA and gait speed using the 4-meter walking test. Additionally handgrip strength by Jamar dynamometer was utilized for EWGSOP and FNIH criteria. 
Table 1: Criteria for low muscle mass, handgrip strength and sarcopenia adapted from the 4 consensus groups (EWGSOP, IWGS, SIG, FNIH).

\begin{tabular}{|c|c|c|c|c|}
\hline Definition & Criteria & Men & Women & Ref \\
\hline \multirow[t]{6}{*}{ EWGSOP } & -Handgrip strength (per BMI category) & $\leq 24: \leq 29 \mathrm{~kg}$ & $\leq 23: \leq 17 \mathrm{~kg}$ & $(1,31)$ \\
\hline & & 24.1-26: $\leq 30 \mathrm{~kg}$ & $23.1-26: \leq 17.3 \mathrm{~kg}$ & \\
\hline & & 26.1-28: $\leq 30 \mathrm{~kg}$ & 26.1-29: $\leq 18 \mathrm{~kg}$ & \\
\hline & & $>28: \leq 32 \mathrm{~kg}$ & $>29: \leq 21 \mathrm{~kg}$ & \\
\hline & -Gait speed & $\leq 0.8 \mathrm{~m} / \mathrm{s}$ & $\leq 0.8 \mathrm{~m} / \mathrm{s}$ & \\
\hline & -SMI & $<8.87 \mathrm{~kg} / \mathrm{m}^{2}$ & $<6.42 \mathrm{~kg} / \mathrm{m}^{2}$ & \\
\hline IWGS & Gait speed $<1 \mathrm{~m} / \mathrm{s}+$ low SMI & $<10.76 \mathrm{~kg} / \mathrm{m}^{2}$ & $<6.76 \mathrm{~kg} / \mathrm{m}^{2}$ & (15) \\
\hline \multirow[t]{2}{*}{ SIG } & Gait speed $<0.8 \mathrm{~m} / \mathrm{s}+$ low RMM & Class $1(<37 \%)$ & Class $1(<28 \%)$ & (32) \\
\hline & & Class $2(<31 \%)$ & Class $2(<22 \%)$ & \\
\hline \multirow[t]{3}{*}{ FNIH } & Weakness + low SMI & & & (6) \\
\hline & - Handgrip strength & $<26 \mathrm{~kg}$ & $<16 \mathrm{~kg}$ & \\
\hline & - SMI & $<8.87 \mathrm{~kg} / \mathrm{m}^{2}$ & $<6.42 \mathrm{~kg} / \mathrm{m}^{2}$ & \\
\hline
\end{tabular}

EWGSOP=European Working Group on Sarcopenia in Older People; IWGS= International Working Group on Sarcopenia; SIG= Special Interest Group of Sarcopenia, Cachexia and Wasting Disorders; FNIH= Foundation for the National Institutes of Health; BMI: Body Mass Index; SMI=absolute skeletal muscle mass /height ${ }^{2}$; RMM= absolute skeletal muscle mass /weight

\section{Patient status 2 years after hospitalization}

Information on each patient's status was retrieved from the hospital electronic medical file, primary care physician and/or patient's primary caregiver, to determine whether patients were still alive or were deceased at 12 and 24 months after the initial hospital admission, including the exact date of death for survival curve analyses. One researcher retrieved all the information at one time point 2 years after the inclusion of the last patient.

\section{Statistics}

Data were analysed using SPSS Statistics version 23 (IBM Corp., Armonk, NY, USA). Patients' characteristics are described by mean $\pm S D$ and range for continuous variables and by frequencies and percentages for the categorical variables. Kaplan Meyer curves and Cox proportional hazard ratio analysis were used to assess the proportional risk of mortality after 1and 2-years for patients with sarcopenia compared to non sarcopenic patients based on the cut-off points of sarcopenia according the consensus criteria of the EWGSOP, IWGS, SIG and FNIH.

A 2-factor ANOVA model (gender $x$ patient status) was used to assess gender-specific differences between 1- and 2-year survivors and non-survivors with respect to: BMI, FFM, 
SMI, RMM, PA, BCM, FMI, SMM/FM, HGS assessed with Jamar dynamometer, GS, SPPB, HABAM, Fried score, GFI, SNAQ score, Katz-ADL score, CIRS score, MMSE and age. Subsequently, Cox proportional hazard ratio analysis was performed to determine which of these variables could best predict 1- and 2-year mortality. Finally, Cox proportional hazard ratio analysis was performed, combining consensus criteria for sarcopenia with the separate patient characteristics to determine whether the calculated hazard ratios are affected (confounded) by different covariates. Because of the limited number of patients included and the limited number of 'events' (i.e., number of deaths throughout the follow up period), a maximum of 3 covariates were tested at the same time. Since no major differences were observed for all analyses in relation to 1-vs 2-year mortality, we focus on presenting 2-year mortality data, referring to supplementary tables for 1-year mortality data for completeness.

\section{Results}

\section{Patient characteristics}

Mean age of the 81 patients included in this study was $84 \pm 5$ y and $73 \%(n=59)$ were female. Seventy eight percent of the patients lived in the community, in the surroundings of the hospital. The CIRS score was 20.0 \pm 5.5 ; a list of main diagnosis at hospital admission and a list of the main co-morbidities present is provided in Supplementary table 1 and 2. Thirty-nine percent of the participants were malnourished, with SNAQ scores of 3 or higher. Forty-seven percent were highly ADL dependent, with a Katz ADL-6 score of 5 or 6.

\section{Consensus criteria and mortality}

The frequency of patients with sarcopenia was different using the different consensus criteria (table 1 and 2). According to the EWGSOP, IWGS, SIG and FNIH the prevalence of sarcopenia in the acutely hospitalized geriatric patients was respectively $51,73,69$, and $27 \%$.

The Kaplan Meyer survival curves showed significantly higher mortality rates for the sarcopenic compared with non-sarcopenic acutely hospitalized geriatric patients according to the consensus criteria of the EWGSOP (73\% versus 25\% 2-year mortality; Figure 1A) and FNIH (90\% versus 36\% 2-year mortality; Figure 1B), but not for the IWGS and SIG (Table 2). Remarkably, 2-year mortality was higher in the non-sarcopenic compared with the sarcopenic patients according to the SIG criteria (68\% versus $41 \%)$. In agreement with the Kaplan Meyer curves, hazard ratios for mortality were significantly higher in sarcopenic patients compared to non-sarcopenic patients when using the EWGSOP (2-y HR 4.310; Cl-95\%: 2.092-8.850; $P<0.001$; Figure 2A) and FNIH criteria (2-y HR 3.571; Cl-95\%: 1.901-6.711; $P<0.001$; Figure 2B) but not for IWGS, while the SIG criteria showed a reduced 2-y HR for sarcopenic vs nonsarcopenic patients (Table 3). Data for 1-year mortality were similar and are provided in Supplementary tables 3-4. 
Table 2: Case summary of sarcopenia according to EWGSOP, IWGS, SIG, FNIH consensus criteria in acutely hospitalized geriatric patients $(n=81)$ and 2-year mortality.

2-year

\begin{tabular}{l|cc|cc}
\cline { 2 - 5 } \multicolumn{2}{c|}{} & \multicolumn{2}{c}{ Non-sarcopenic } & \multicolumn{2}{c}{ Sarcopenic } \\
\cline { 2 - 5 } \multicolumn{1}{c|c}{ Alive } & Dead & $11(27 \%)$ & $30(73 \%)$ \\
\hline IWGS & $30(75 \%)$ & $10(25 \%)$ & $27(46 \%)$ & $32(54 \%)$ \\
SIG & $14(64 \%)$ & $8(36 \%)$ & $33(59 \%)$ & $23(41 \%)$ \\
FNIH & $8(32 \%)$ & $17(68 \%)$ & $3(14 \%)$ & $19(86 \%)$ \\
\hline
\end{tabular}

Data represent the absolute number (and the \%) of patients who deceased and were alive after 2 years according to EWGSOP, IWGS, SIG, FNIH consensus criteria of sarcopenia.

EWGSOP=European Working Group on Sarcopenia in Older People; IWGS= International Working Group on

Sarcopenia; SIG= Special Interest Group of Sarcopenia, Cachexia and Wasting Disorders; FNIH= Foundation for the National Institutes of Health

Body composition parameters and 2-year mortality

To assess the association of various characteristics, both sarcopenia-related and not sarcopenia-related, with mortality, comparisons were made between patients that did and did not survive after 2 years (Table 4 ).

The geriatric patients who deceased within 2 years after initial hospitalization were significantly $(P<0.05)$ older, and had a lower BMl compared to the patients who were still alive. The Relative Muscle Mass (RMM) and Fat Free Mass (FFM) were significantly lower in women vs men, but there was no difference in RMM and FFM between the patients who deceased and those that were still alive. In contrast, skeletal muscle mass index (SMI) was significantly $(P<0.05)$ lower both in women vs men, and in the geriatric patients who deceased compared to those who survived.

Patients who deceased had a significantly lower Phase Angle (PA) compared to the patients who were alive 2 years after initial hospital admission, with no significant gender difference (2y: PA women: $6.2 \pm 1.3$ vs $7.0 \pm 1.4$; PA men: $6.0 \pm 1.6$ versus $7.4 \pm 1.7$; all $P<0.05$ ). However the PA was higher in men who were still alive compared to women. Body Cell Mass (BCM) was significantly higher $(P<0.05)$ in men compared to women and was significantly lower in the geriatric patients who were deceased after 2 years compared to those who survived.

In line with $\mathrm{BMI}$ and SMI, the fat mass index (FMI) was different between men and women, and FMI was significantly lower in the geriatric patients who had deceased after 2 years compared to the patients who were still alive (2-y: FMI women: $8.0 \pm 3.8$ vs $11.2 \pm 4.3$; FMI men: $5.7 \pm 2.4$ vs $7.8 \pm 3.2 \mathrm{~kg} / \mathrm{m}^{2}$; all $\mathrm{P}<0.05$ ). Findings for fat mass percentage were in agreement with FMI. 

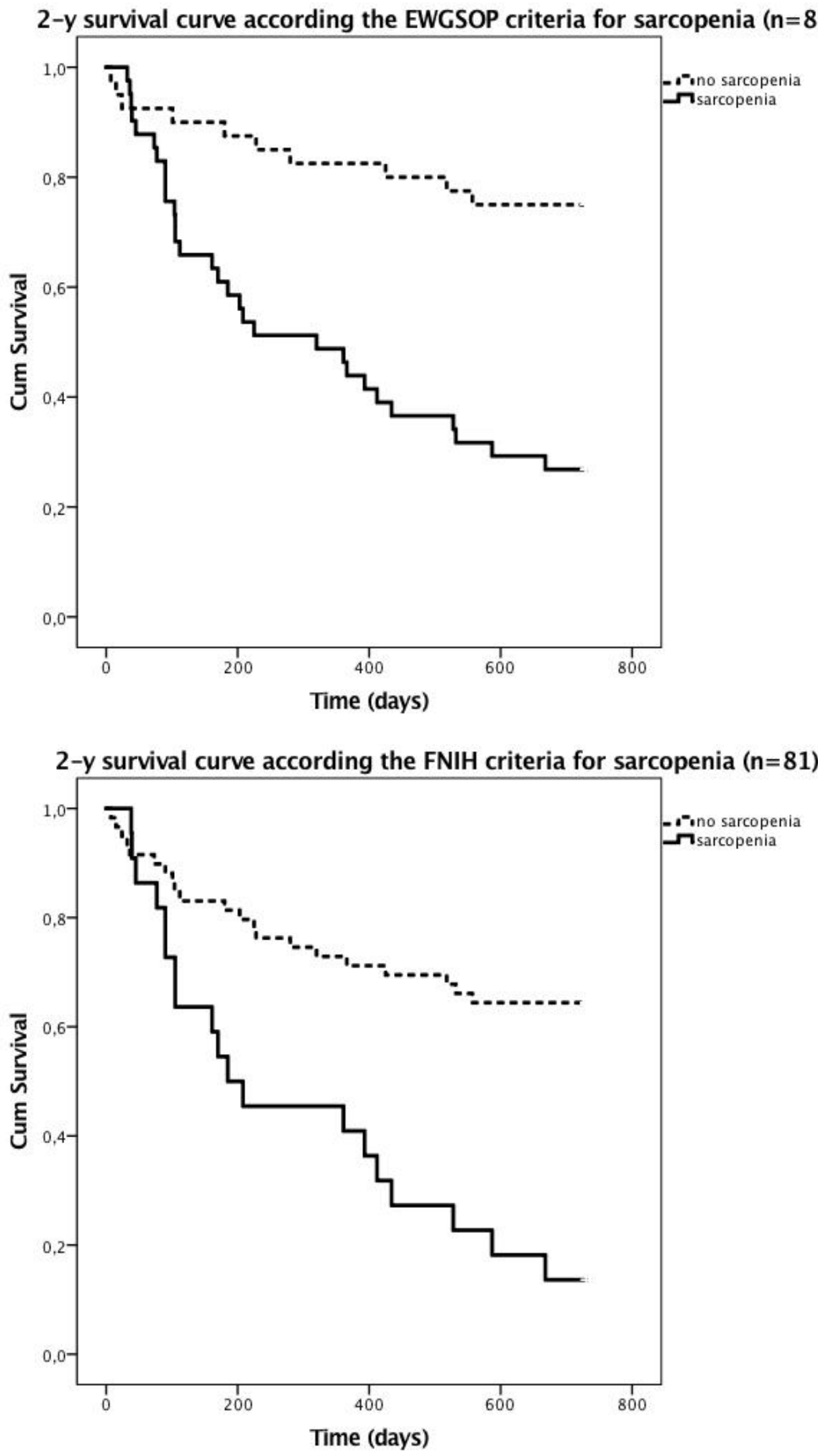

Figure 1: Kaplan Meier Survival curve for acutely hospitalized geriatric patients with or without sarcopenia according the EWGSOP (A (top); 2-year mortality, $P<0.001)$ and FNIH (B (bottom); 2-year mortality, $P<0.001)$. EWGSOP=European Working Group on Sarcopenia in Older People; FNIH= Foundation for the National Institutes of Health 

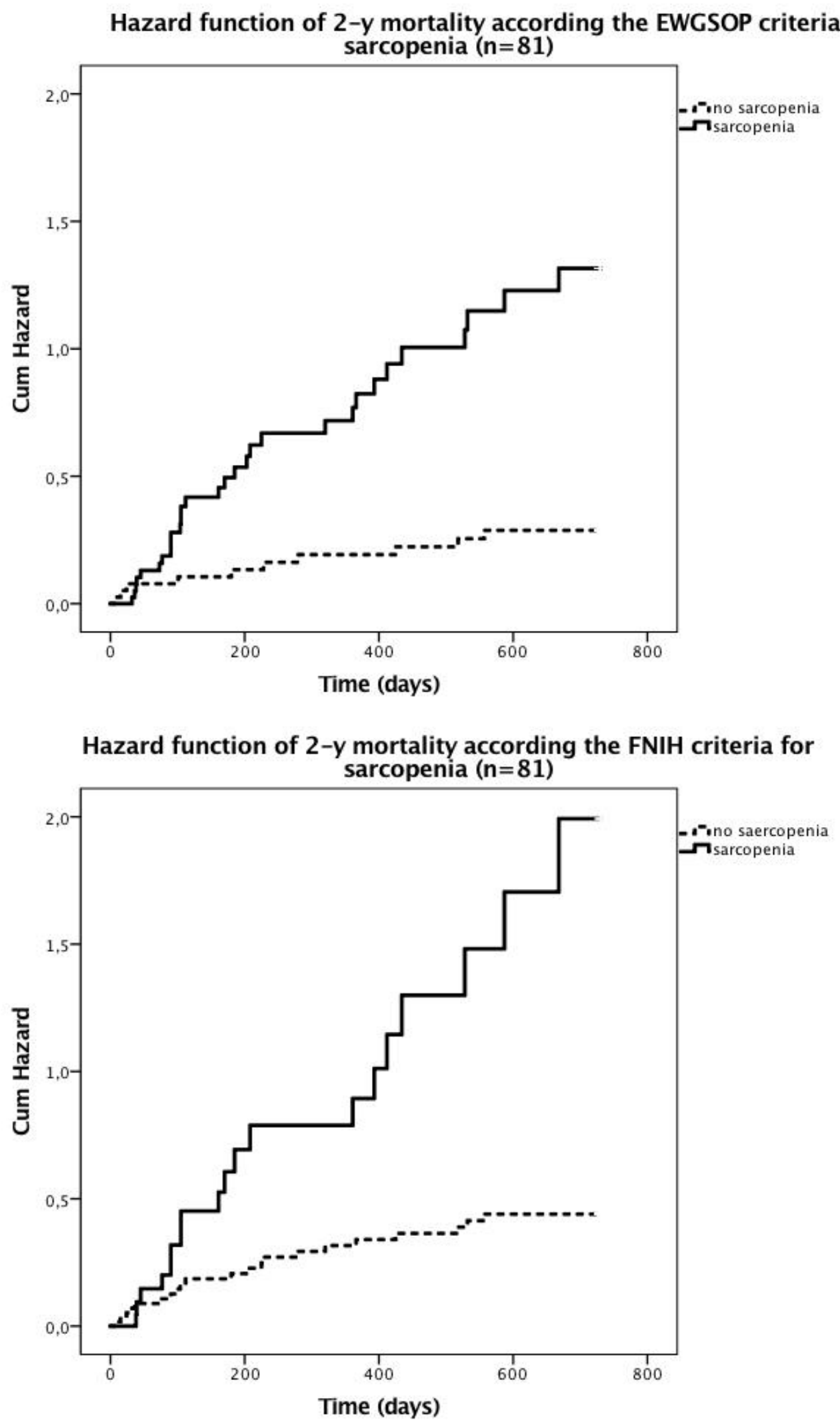

Figure 2: Hazard function curve of 2-y mortality for acutely hospitalized geriatric patients with or without sarcopenia according the EWGSOP (A (top); 2-year mortality, $P<0.001$ ) and FNIH (B (bottom); 2-year mortality, $P<0.001)$. EWGSOP=European Working Group on Sarcopenia in Older People; FNIH= Foundation for the National Institutes of Health 
The skeletal muscle mass-fat mass ratio (SMM/FM) was significantly lower in women compared to men and was significantly higher in the patients who were deceased after 2 years compared to the patients who survived (Table 4).

Table 3: Cox proportional hazard ratio of 2-y mortality for acutely hospitalized geriatric patients with sarcopenia vs. no sarcopenia according the EWGSOP, IWGS, SIG and FNIH criteria for sarcopenia $(n=81)$.

\begin{tabular}{l|ccccc} 
& no sarcopenia & sarcopenia & HR & CI -95\% & $\boldsymbol{P}$ \\
\hline EWGSOP & 40 & 41 & 4.310 & $2.092-8.850$ & $<0.001^{*}$ \\
IWGS & 22 & 59 & 1.754 & $0.808-3.817$ & 0.155 \\
SIG & 25 & 56 & 0.488 & $0.260-0.916$ & $0.025^{*}$ \\
FNIH & 59 & 22 & 3.571 & $1.901-6.711$ & $<0.001^{*}$
\end{tabular}

Data represent the Cox proportional hazard ratio of mortality in acutely hospitalized geriatric patients with sarcopenia compared with no sarcopenia after 2 years according to EWGSOP, IWGS, SIG, FNIH consensus criteria of sarcopenia.

EWGSOP=European Working Group on Sarcopenia in Older People; IWGS= International Working Group on Sarcopenia; SIG= Special Interest Group of Sarcopenia, Cachexia and Wasting Disorders; FNIH= Foundation for the National Institutes of Health

*significantly different hazard ratio between patients with or without sarcopenia

Muscle strength and physical function versus 2-year mortality

The maximal handgrip strength was significantly lower in women compared to men. Handgrip strength was not significantly different for the geriatric patients who had deceased after 2 years compared to the patients who survived (Table 4).

Gait speed (GS) was significantly lower in women compared to men. In addition, GS was significantly lower in the geriatric patients that died within 2 years compared with those patients who survived (GS women: $0.39 \pm 0.12$ vs $0.51 \pm 0.23 \mathrm{~m} / \mathrm{s}$; GS men: $0.47 \pm 0.21$ vs 0.79 $\pm 0.44 \mathrm{~m} / \mathrm{s})$. Almost all geriatric patients $(n=77)$ had low physical performance with a SPPB score <4. SPPB was significantly lower in women compared to men. However, there was no significant difference in physical performance according the SPPB in the patients who deceased and those who survived after 2 years. Likewise, physical performance according the HABAM was not significantly different between geriatric patients who deceased and survived within 2 years (Table 4).

Frailty, nutrition, $A D L$, comorbidity and age versus 2-year mortality

The geriatric patients who deceased within 2 years were more frail according to the Fried score compared with the patients who were still alive, but this was not confirmed according to the GFI. There was no significant difference in nutritional status (SNAQ), care dependency 
(Katz-ADL), comorbidity (CIRS) and cognitive function (MMSE) between the geriatric patients who had deceased or were still alive after2 years (Table 4).

Table 4: Body composition, muscle strength physical function, frailty, nutrition, $A D L$, comorbidity and age versus 2-year survival in acutely hospitalized geriatric patients $(n=81)$.

Women ( $\mathbf{n = 5 9 )}$ Men $(\mathbf{n}=22)$

$\begin{array}{lllllll}\text { Deceased } & n & \text { Alive } & \text { D } & \text { Alive } & \\ \end{array}$

\section{General}

\begin{tabular}{lllllllll}
\hline Age, $\mathbf{y}$ & $86.0 \pm 5.4$ & 29 & $83.4 \pm 5.1^{*}$ & 30 & $84.8 \pm 7.4$ & 11 & $82.7 \pm 6.4^{*}$ & 11 \\
BMI, $\mathbf{k g} / \mathrm{m}^{2}$ & $23.4 \pm 4.7$ & 29 & $26.7 \pm 4.8^{*}$ & 30 & $23.2 \pm 4.7$ & 11 & $25.9 \pm 4.0^{*}$ & 11 \\
\hline
\end{tabular}

Body composition

\begin{tabular}{lllllllll}
\hline FFM, kg & $38.1 \pm 5.9^{\#}$ & 29 & $39.6 \pm 54.2^{\#}$ & 30 & $48.1 \pm 7.6$ & 11 & $52.4 \pm 8.4$ & 11 \\
SMI, kg/m & $6.3 \pm 0.7^{\#}$ & 29 & $6.6 \pm 0.4^{* \#}$ & 30 & $7.6 \pm 1.0$ & 11 & $8.5 \pm 0.8^{*}$ & 11 \\
RMM, \% & $27.6 \pm 3.8^{\#}$ & 29 & $25.4 \pm 3.2^{\#}$ & 30 & $34.3 \pm 3.1$ & 11 & $33.3 \pm 4.0$ & 11 \\
Phase angle & $6.2 \pm 1.3$ & 29 & $7.0 \pm 1.4^{*}$ & 30 & $6.0 \pm 1.6$ & 11 & $7.4 \pm 1.7^{*}$ & 11 \\
BCM, kg & $19.6 \pm 2.7^{\#}$ & 29 & $20.5 \pm 2.0^{* \#}$ & 30 & $24.6 \pm 2.8$ & 11 & $27.6 \pm 3.9^{*}$ & 11 \\
FMI, kg/m & $8.0 \pm 3.8^{\#}$ & 29 & $11.2 \pm 4.3^{* \#}$ & 30 & $5.7 \pm 2.4$ & 11 & $7.8 \pm 3.2^{*}$ & 11 \\
FM\% & $33.2 \pm 8.8^{\#}$ & 29 & $40.6 \pm 8.1^{* \#}$ & 30 & $24.3 \pm 6.0$ & 11 & $30.4 \pm 7.3^{*}$ & 11 \\
SMM/FM & $1.0 \pm 0.4^{\#}$ & 29 & $0.7 \pm 0.3^{* \#}$ & 30 & $1.5 \pm 0.4$ & 11 & $1.2 \pm 0.4^{*}$ & 11 \\
\hline
\end{tabular}

\section{Physical function}

\begin{tabular}{lllllllll}
\hline HGS Jamar, kg & $14.8 \pm 5.3^{\#}$ & 29 & $16.4 \pm 6.0^{\#}$ & 30 & $24.2 \pm 5.5$ & 11 & $26.7 \pm 8.0$ & 11 \\
GS, m/s & $0.39 \pm 0.12^{\#}$ & 18 & $0.51 \pm 0.23^{\star \#}$ & 22 & $0.47 \pm 0.21$ & 11 & $0.79 \pm 0.44^{*}$ & 10 \\
SPPB & $2.2 \pm 1.9^{\#}$ & 29 & $3.0 \pm 2.6^{\#}$ & 30 & $4.1 \pm 2.4$ & 11 & $4.6 \pm 3.3$ & 11 \\
HABAM & $35.6 \pm 11.9$ & 29 & $35.4 \pm 15.5$ & 30 & $39.5 \pm 10.7$ & 11 & $43.9 \pm 15.0$ & 11
\end{tabular}

Frailty, nutrition, ADL, comorbidity and cognitive function

\begin{tabular}{lllllllll}
\hline Fried score & $4.1 \pm 0.7$ & 29 & $3.8 \pm 0.6^{*}$ & 30 & $4.0 \pm 0.6$ & 11 & $3.5 \pm 0.8^{*}$ & 11 \\
GFI & $8.2 \pm 2.8$ & 29 & $7.2 \pm 2.5$ & 28 & $8.2 \pm 2.6$ & 11 & $6.7 \pm 2.4$ & 11 \\
SNAQ & $2.2 \pm 1.8$ & 29 & $1.5 \pm 1.5$ & 28 & $1.9 \pm 1.8$ & 11 & $2.1 \pm 1.3$ & 11 \\
Katz-ADL & $3.8 \pm 2.0$ & 29 & $3.4 \pm 2.2$ & 28 & $3.5 \pm 2.3$ & 11 & $4.0 \pm 1.5$ & 11 \\
CIRS & $20.2 \pm 5.2$ & 29 & $18.5 \pm 5.0$ & 30 & $21.7 \pm 5.0$ & 11 & $20.5 \pm 6.7$ & 11 \\
MMSE & $19.1 \pm 5.6$ & 23 & $20.4 \pm 5.6$ & 24 & $18.1 \pm 5.2$ & 11 & $21.4 \pm 5.2$ & 11
\end{tabular}

Data are means \pm SD. BMI: Body Mass Index; FFM: Fat Free Mass; SMI: Skeletal Muscle Mass Index; RRM: Relative Muscle Mass; BCM: Body Cell Mass; FMI: Fat Mass Index; FM\%: Fat Mass Percentage; SMM/FM: Skeletal Muscle Mass/Fat Mass; HGS Jamar: Handgrip Strength measured with Jamar dynamometer; GS: Gait Speed; SPPB: Short Physical Performance Battery; HABAM: Hierarchical Balance and Mobility; GFI: Groningen Frailty Indicator; SNAQ: Short Nutritional Assessment Questionnaire; CIRS: Cumulative IIIness Rating Scale; MMSE: Minimal Mental State Examination; ${ }^{*}$ significantly different from deceased $(P<.05)$; \#significantly different from men $(P<.05)$ 
For all patient characteristics described above for 2 years survival, similar differences were observed between patients that had survived vs those that were deceased after 1 year (see supplementary Table 5 for details).

Table 5: Hazard Ratios for potential predictors for 2-y mortality in hospitalized geriatric patients $(n=81)$ with additional analysis including gait speed $(n=61)$

2-y mortality probability

\begin{tabular}{l|ccccc}
\hline & & $\mathrm{n}$ & HR & Cl-95\% & $P$ \\
\hline Step 1 & FMI & 81 & 0.841 & $0.761-0.931$ & $0.001^{*}$ \\
Step 2 & PA & & 0.678 & $0.531-0.864$ & $0.002^{*}$ \\
& FMI & 0.839 & $0.758-0928$ & $0.001^{*}$ \\
\hline Step 1 & FMI & 61 & 0.826 & $0.729-0.936$ & $0.003^{*}$ \\
Step 2 & PA & 0.675 & $0.512-0.891$ & $0.005^{*}$ \\
Step 3 & FMI & 0.835 & $0.734-0.949$ & $0.006^{*}$ \\
& PA & 0.712 & $0.532-0.954$ & $0.023^{*}$ \\
& FMI & 0.825 & $0.723-0.940$ & $0.004^{*}$ \\
\hline
\end{tabular}

Data represent the results of Cox proportional hazard analysis for FMI, PA and SMI for 2-year mortality in acutely hospitalized geriatric patients $(n=81)$. Additionally Cox proportional hazard mortality analysis were performed for the patients with available gait speed at hospital admission (n=61) FMI: Fat Mass Index; PA: Phase Angle; GS: Gait Speed. *significant $(P<.05)$

\section{Cox proportional hazard ratio}

Based on the ANOVA results described above, BMI, SMI, PA, BCM, FMI, SMM/FM, Fried score, and age were included as potential predictors for 2-year mortality in a Cox proportional hazard ratio model. Cox proportional hazard ratio analysis was performed on data for $n=81$ geriatric patients. Patients with higher PA (HR 0.678; $\mathrm{Cl}-95 \%: 0.531-0.864 ; P=0.002)$ and higher FMI (HR 0.839; Cl-95\%: 0.758-0.928; $P=0.001$ ) had a significantly lower mortality probability throughout the 2 year follow-up after hospital admission (Table 5).

For a subgroup of geriatric patients $(n=61)$ gait speed was available, and was added to the Cox proportional hazard ratio analyses. Patients with higher PA (HR 0.712; Cl-95\%:0.5320.954; $P=0.023$ ), higher FMI (HR 0.825; Cl-95\%: 0.723-0.940; $P=0.004)$ and higher gait speed (HR 0.085; Cl-95\%: 0.010-0.729; $P=0.025$ ) had a significantly lower 2-y mortality probability (Table 5). Again, similar findings were observed for 1-year mortality (Supplementary Table 6).

As a final step in the analysis, we assessed whether the HRs for the presence of sarcopenia according the EWGSOP and FNIH criteria were affected by correcting for other variables. Therefore, the following patient characteristics were separately added as covariates in the Cox proportional hazard ratio analysis: age, gender, BMI, Katz-ADL, SNAQ, CIRS and MMSE. None of these variables was associated with mortality probability (HR not different from 1), and 
none of these variables changed the hazard ratio for the presence of sarcopenia according the EWGSOP and FNIH criteria. Only GFI was found to be independently associated with mortality probability. Therefore, in the final model, inclusion of sarcopenia criteria was combined with the inclusion of PA, FMI, and GFI, as these were all shown to be (separately) affecting the mortality hazard ratio. 2-y mortality probability was reduced in patients with higher PA (HR 0.699; Cl-95\%:0.546-0.895; $P=0.005)$, and increased in patients with higher GFI (HR 1.120; Cl-95\%: 1.001-1.254; $P=0.049$ ), and in patients with sarcopenia according the EWGSOP criteria, with essentially unaltered HR (HR 4.040; Cl-95\%:1.960-8.239; $P<0.001$ ) compared to the unadjusted model (Table 3). FMI was no longer associated with 2-y mortality probability when corrected for the other variables (HR 0.924; Cl-95\%:0.812-1.052; $P=0.232$ ). When the model was ran using the FNIH criteria for sarcopenia, 2-y mortality was reduced in patients with higher PA (HR 0.679; Cl-95\%:0.527-0.874; $P=0.003$ ), and increased in patients with sarcopenia, with essentially unaltered HR (HR 3.433; Cl-95\%:1.822-6.506; $P<0.001)$ compared to the unadjusted model (Table 3).

\section{Discussion}

In this study we demonstrate that sarcopenia was highly prevalent in older patients admitted to the acute geriatric ward, but varied widely (27-73\%) when different sarcopenia criteria were used. Only sarcopenia according to the EWGSOP and the FNIH criteria was significantly associated with up to 4.3 times higher mortality probability compared to non-sarcopenic patients Additionally several hallmarks of sarcopenia and other patient characteristics, including skeletal muscle mass index, fat mass index, body cell mass, body mass index, phase angle and gait speed, were significantly lower in the geriatric patients who had deceased after 2 years compared to the patients who were still alive. Cox proportional hazard ratio analysis showed that higher gait speed, phase angle, and fat mass index are associated with reduced 2-year mortality probability in these hospitalized geriatric patients. However when correcting for various covariates, mortality probability remained strongly associated with sarcopenia according EWGSOP and FNIH criteria, with phase angle significantly adding to the model.

As expected, the prevalence of sarcopenia was high in our population of hospitalized geriatric patients. In accordance with results from the Leiden Longevity Study however, sarcopenia prevalence varied substantially when different criteria were used (33). Given the recent recognition of sarcopenia as a medical condition with its own ICD-10 CM code (M62.84), there is a clear need for well defined and generally acknowledged criteria for sarcopenia $(34,35)$. Indeed, to enable better comparison between studies, to specify prevalence rates, and to better target those in need of treatment, further consensus has to be reached on the exact 
diagnostic criteria and cut-off values for sarcopenia.

Apart from clearly establishing the diagnosis of sarcopenia, consensus criteria need to have power to predict adverse outcome like mortality. For this reason we studied the predictive value of different diagnostic criteria for sarcopenia and, subsequently, individual parameters of physical function and body composition for mortality. When applying the criteria of sarcopenia according to the different consensus groups, only the EWGSOP and FNIH criteria were significantly associated with an increased 2-year mortality in sarcopenic vs non-sarcopenic patients. Until now, mortality has only been studied up to 3-12 months after hospital admission. In accordance with our findings, Cerri and colleagues (12) previously found an increased 3-month mortality rate in hospitalized malnourished geriatric patients applying the EWGSOP algorithm. Average gait speed and SMI was higher in their study when compared to our findings. Additionally the study of Perez-Zepeda and co-workers (13) showed a comparable increased 1 year mortality in sarcopenic geriatric patients applying EWGSOP criteria. However their study population was different from our population because they excluded patients with delirium and dementia and measurement was done within 6 days after hospital admission after transfer from an acute medical unit. Besides that, cut off values for skeletal muscle mass and gait speed were different from the original EWGSOP algorithm (13). In contrast to these findings of increased mortality up to 2 years after hospitalization in sarcopenic vs non-sarcopenic geriatric patients, sarcopenic patients according to the SIG criteria had a better 2-year survival compared to the non-sarcopenic patients. One of the hallmarks in the SIG criteria is relative skeletal muscle mass (RMM), which means skeletal muscle mass divided by body mass. Low RMM can be apparent when skeletal muscle mass is normal, but body mass is (relatively) high as a consequence of increased fat mass. Likewise, 'normal' RMM (and thus 'no sarcopenia') could be associated with low skeletal muscle mass in the combination with even lower total body mass. As such, truly cachectic patients (who probably have a higher mortality) may be defined as non-sarcopenic, whereas 'overweight' patients with a normal muscle mass may be defined as sarcopenic when using the SIG criteria. This likely explains the contradictory relation with mortality observed in the present study. Indeed, previous work has also described a partly protective effect of minor overweight in older people (36), at least partly explaining our findings. In agreement, we show in the present study that the patients who had survived after 2 years had a higher fat mass index compared with those who had died.

Overall, the geriatric patients in our study were extremely frail, with mean handgrip strength and mean gait speed far below the cut-off values of the different consensus criteria. This homogeneity in physical performance below cut-off values likely resulted in poor discriminative 
potential of the sarcopenia criteria according IWGS to predict mortality within our population of frail acutely hospitalized geriatric patients.

Because of the huge differences in prevalence and difference of association of sarcopenia between the different consensus criteria and mortality, we next studied individual parameters of sarcopenia like body composition and physical function, rather than only differentiating between sarcopenic and non-sarcopenic. We show that apart from skeletal muscle mass index and gait speed (i.e., sarcopenia associated parameters), also phase angle, body cell mass, and fat mass index/percentage were significantly different between the geriatric patients who deceased and those who were alive after 2 years. Low skeletal muscle mass in combination with low handgrip strength or low gait speed has previously been associated with an increased mortality in hospitalized elderly patients $(12,37)$. The phase angle is a marker of overall cell and tissue vitality (38). The association between phase angle and mortality in geriatric patients is in agreement with earlier observations in cancer patients (38), as well as in a communitydwelling population of older adults (39). Also in line with our findings, Bouillanne and coworkers have shown that increased fat mass is associated with decreased adverse outcome like mortality in hospitalized elderly patients (40). In the present study, gait speed was very low and, on average, far below the cut off values of the different consensus criteria. However, when studied as an individual parameter, gait speed was still significantly lower in the geriatric patients who deceased after 2-years compared to those who survived. In acute care settings, lower gait speed $(0.46 \mathrm{~m} / \mathrm{s})$ was found in patients aged $70 \mathrm{y}$ and older compared with gait speed recorded in outpatient settings $(0.74 \mathrm{~m} / \mathrm{s})(41)$. In agreement with earlier studies, gait speed is a strong predictor of mortality (42), however in a recent review this was only confirmed for men (43). Handgrip strength was far below the cut off point in the EWGSOP criteria but not significantly lower in the geriatric patients who had deceased after 2 years. The widely used screening tests for frailty (Fried, GFI), malnutrition (SNAQ), functional decline (Katz-ADL), comorbidity (CIRS) and cognitive function (MMSE) were not associated with mortality in this frail geriatric population. Taking these findings all together, only parameters of physical function and body composition seem to be associated with mortality in these hospitalized geriatric patients, with no major differences in their relation with 2 year mortality.

To truly determine which of the parameters that differed between survivors and non-survivors could predict mortality in these hospitalized geriatric patients we performed Cox proportional hazard ratio analysis. Based on the hazard ratio's shown in Table 5 (and Supplementary table 6 ), we clearly showed that the combination of phase angle and FMI could best predict mortality risk. For example, mortality risk at any given point in time throughout the 2-yr period after hospital admission was $47.5 \%$ lower with each unit increase in phase angle, and $19.2 \%$ lower 
with each $\mathrm{kg} / \mathrm{m}^{2}$ increase in fat mass. In the subgroup of patients for which gait speed data were available, mortality risk throughout the 2-yr period after hospital admission was $40.4 \%$ lower with each unit increase in phase angle, $21.2 \%$ lower with each $\mathrm{kg} / \mathrm{m}^{2}$ increase in fat mass, and $17.6 \%$ lower for each $0.1 \mathrm{~m} / \mathrm{s}$ increase in gait speed. Based on the final regression models in which we combined both sarcopenia (EWGSOP or FNIH) and the separate patient characteristics, thus correcting for several covariates, mortality probability remained strongly associated with sarcopenia, with phase angle significantly adding to the model. Though generalization of these findings should obviously be done with caution given the relatively small number of patients included in this study, our findings strongly indicate that certain physical characteristics -that are not necessarily used in the assessment of sarcopenia- are predictive for overall mortality in acutely hospitalized geriatric patients and, as such, may represent relevant diagnostic tools that may be taken into account when determining the treatment plan of these patients.

The current study was a single centre study, limited to one acute care geriatric ward of a Dutch general hospital and we only included geriatric patients who were mobile prior to hospitalization and were (cognitively) able to follow our study instructions. As such, we included a relatively small number of patients and could only adjust our analyses for a limited number of covariates. It is thus difficult to generalize our findings to the overall population of acutely hospitalized geriatric patients. Furthermore, we had missing values for $33 \%$ of the eligible patients. Although age and physically frailty in these patients was comparable to the included patients (data not shown), we cannot exclude potential confounding effects of this substantial 'dropout'. It does however support the notion that it is extremely difficult to include these type of patients in this type of research. As a third limitation, gait speed was lacking in almost 25\% $(n=20)$ patients at hospital admission and could therefore influence sarcopenia classification. These patients were too weak to walk at hospital admission. However, we performed a 4-meter gait speed test one week later and gait speed was in all 20 patients below $0.8 \mathrm{~m} / \mathrm{s}$ (data not shown). As such, risk for misclassification was minimal, as the initial lack of gait speed data did not influence classification of these patients into sarcopenic or nonsarcopenic.

As a final limitation, we only used body composition data from the BIA assessment and thus modified the original sarcopenia criteria of the IWGS and FNIH by replacing aSM by SMI. Although it is generally acknowledged that $\sim 75 \%$ of total muscle mass consists of aSM (30, 44), and cut-offs for SMI were based on previous reports (45), the replacement of aSM with BIA-based SMI data has in itself not been validated and may have slightly impacted the sarcopenia definition. Also, bio-impedance measures such as used in our work are affected by 
the hydration status of patients, and changes herein (e.g. dehydration, edema) are notorious in geriatric patients. This issue is inherent to the population studied and also affects MRI or DXA based assessments. Currently, there is no valid manner to account for this potential confounding effect. In general though, prediction equations for muscle mass based on BIA have been well validated against MRI data $(20,44)$, supporting its use for both research and clinical practice. Moreover, in daily clinical practice of a geriatric ward, the use of BIA is much more realistic than DXA or MRI scans. Indeed, bio-impedance represents an easy accessible tool for measuring body composition with substantial predictive power for mortality, which could be of considerable value in clinical practice. This may be especially the case in targeting medical treatment in relation to geriatric patients with very limited life expectancy. Based on our findings, it could be valuable for the daily practice of a geriatrician to assess gait speed and body composition with bio impedance analysis for skeletal muscle mass, fat mass, and phase angle to identify those patients with an increased mortality risk. This may be especially relevant when a decision should be made when a medical treatment with huge impact is considered in hospitalized geriatric patients. However our study results should first be confirmed in larger clinical trials, including more centres and representing a larger spectrum of the total population of acutely hospitalized geriatric patients, also enabling the adjustment of potential relevant covariates, and including other relevant parameters such as physical functioning or readmission rates, before concrete clinical implementation is in order.

In conclusion, we show that prevalence of sarcopenia in acutely hospitalized geriatric patients is highly dependent on the criteria used. Sarcopenia according the EWGSOP and FNIH criteria is highly present and is associated with increased 2-y mortality in acutely hospitalized geriatric patients. Mortality probability is also predicted by variables like phase angle and fat mass. However when correcting for several confounders, mortality probability is best predicted by the combination of sarcopenia and phase angle. We propose that systematic bioimpedance based assessment of sarcopenia and phase angle could be of additional value in daily practice of geriatric hospital care. 


\section{References}

1. Cruz-Jentoft AJ, Baeyens JP, Bauer JM, Boirie Y, Cederholm T, Landi F, et al. Sarcopenia: European consensus on definition and diagnosis: Report of the European Working Group on Sarcopenia in Older People. Age Ageing. 2010;39(4):412-23.

2. Rosenberg I. Summary comments: epidemiological and methodological problems in determining nutritional status of older persons. . American Journal Clinical Nutrition. 1989;50:1231-3.

3. Evans W. Functional and metabolic consequences of sarcopenia. The Journal of nutrition. 1997;127(5 Suppl):998S-1003S.

4. Hirani V, Blyth F, Naganathan V, Le Couteur DG, Seibel MJ, Waite LM, et al. Sarcopenia Is Associated With Incident Disability, Institutionalization, and Mortality in Community-Dwelling Older Men: The Concord Health and Ageing in Men Project. J Am Med Dir Assoc. 2015;16(7):607-13.

5. Arango-Lopera VE, Arroyo P, Gutierrez-Robledo LM, Perez-Zepeda MU, Cesari M. Mortality as an adverse outcome of sarcopenia. J Nutr Health Aging. 2013;17(3):25962.

6. Dam TT, Peters KW, Fragala M, Cawthon PM, Harris TB, McLean R, et al. An evidence-based comparison of operational criteria for the presence of sarcopenia. J Gerontol A Biol Sci Med Sci. 2014;69(5):584-90.

7. Cruz-Jentoft AJ, Landi F, Schneider SM, Zuniga C, Arai H, Boirie Y, et al. Prevalence of and interventions for sarcopenia in ageing adults: a systematic review. Report of the International Sarcopenia Initiative (EWGSOP and IWGS). Age Ageing. 2014;43(6):74859.

8. Sousa AS, Guerra RS, Fonseca I, Pichel F, Amaral TF. Sarcopenia and length of hospital stay. Eur J Clin Nutr. 2016;70(5):595-601.

9. Sipers WM, Meijers JM, van Dijk RB, Halfens RJ, Schols JM. Impact of Different Diagnostic Criteria on the Prevalence of Sarcopenia in an Acute Care Geriatric Ward. J Frailty Aging. 2014;3(4):222-9.

10. Martinez BP, Batista AK, Gomes IB, Olivieri FM, Camelier FW, Camelier AA. Frequency of sarcopenia and associated factors among hospitalized elderly patients. BMC Musculoskelet Disord. 2015;16:108.

11. Smoliner C, Sieber CC, Wirth R. Prevalence of sarcopenia in geriatric hospitalized patients. J Am Med Dir Assoc. 2014;15(4):267-72.

12. Cerri AP, Bellelli G, Mazzone A, Pittella F, Landi F, Zambon A, et al. Sarcopenia and malnutrition in acutely ill hospitalized elderly: Prevalence and outcomes. Clin Nutr. 2015;34(4):745-51.

13. Perez-Zepeda MU, Sgaravatti A, Dent E. Sarcopenia and post-hospital outcomes in older adults: A longitudinal study. Arch Gerontol Geriatr. 2017;69:105-9.

14. Woo J, Leung J, Morley JE. Validating the SARC-F: a suitable community screening tool for sarcopenia? J Am Med Dir Assoc. 2014;15(9):630-4.

15. Fielding RA, Vellas B, Evans WJ, Bhasin S, Morley JE, Newman AB, et al. Sarcopenia: an undiagnosed condition in older adults. Current consensus definition: prevalence, etiology, and consequences. International working group on sarcopenia. J Am Med Dir Assoc. 2011;12(4):249-56.

16. Brown JC, Harhay MO, Harhay MN. Sarcopenia and mortality among a populationbased sample of community-dwelling older adults. J Cachexia Sarcopenia Muscle. 2016;7(3):290-8.

17. Hoogendijk EO, van Hout HP. Investigating measurement properties of the Groningen Frailty Indicator: a more systematic approach is needed. J Am Med Dir Assoc. 2012;13(8):757; author reply -8.

18. Fried LP, Tangen CM, Walston J, Newman AB, Hirsch C, Gottdiener J, et al. Frailty in older adults: evidence for a phenotype. J Gerontol A Biol Sci Med Sci. 2001;56(3):M146-56.

19. Malnutrition Advisory Group BAPEN. Malnutrition Universal Screening Tool. 2008. p. 11,2 . 
20. Ling $\mathrm{CH}$, de Craen AJ, Slagboom PE, Gunn DA, Stokkel MP, Westendorp RG, et al. Accuracy of direct segmental multi-frequency bioimpedance analysis in the assessment of total body and segmental body composition in middle-aged adult population. Clin Nutr. 2011;30(5):610-5.

21. Lukaski HC. Evolution of bioimpedance: a circuitous journey from estimation of physiological function to assessment of body composition and a return to clinical research. Eur J Clin Nutr. 2013;67 Suppl 1:S2-9.

22. Chien MY, Huang TY, Wu YT. Prevalence of sarcopenia estimated using a bioelectrical impedance analysis prediction equation in community-dwelling elderly people in Taiwan. J Am Geriatr Soc. 2008;56(9):1710-5.

23. Bechtol CO. Grip test; the use of a dynamometer with adjustable handle spacings. The Journal of bone and joint surgery American volume. 1954;36-A(4):820-4; passim.

24. Roberts HC, Denison HJ, Martin HJ, Patel HP, Syddall H, Cooper C, et al. A review of the measurement of grip strength in clinical and epidemiological studies: towards a standardised approach. Age Ageing. 2011;40(4):423-9.

25. Oldfield RC. The assessment and analysis of handedness: the Edinburgh inventory. Neuropsychologia. 1971;9(1):97-113.

26. Fisher S, Ottenbacher KJ, Goodwin JS, Graham JE, Ostir GV. Short Physical Performance Battery in hospitalized older adults. Aging clinical and experimental research. 2009;21(6):445-52.

27. Volpato S, Cavalieri M, Sioulis F, Guerra G, Maraldi C, Zuliani G, et al. Predictive value of the Short Physical Performance Battery following hospitalization in older patients. The journals of gerontology Series A, Biological sciences and medical sciences. 2011;66(1):89-96.

28. MacKnight C, Rockwood K. A Hierarchical Assessment of Balance and Mobility. Age and ageing. 1995;24(2):126-30.

29. Hubbard RE, Eeles EM, Rockwood MR, Fallah N, Ross E, Mitnitski A, et al. Assessing balance and mobility to track illness and recovery in older inpatients. Journal of general internal medicine. 2011;26(12):1471-8.

30. Gallagher D, Visser M, De Meersman RE, Sepulveda D, Baumgartner RN, Pierson RN, et al. Appendicular skeletal muscle mass: effects of age, gender, and ethnicity. J Appl Physiol (1985). 1997;83(1):229-39.

31. Bianchi L, Ferrucci L, Cherubini A, Maggio M, Bandinelli S, Savino E, et al. The Predictive Value of the EWGSOP Definition of Sarcopenia: Results From the InCHIANTI Study. J Gerontol A Biol Sci Med Sci. 2016;71(2):259-64.

32. Muscaritoli M, Anker SD, Argiles J, Aversa Z, Bauer JM, Biolo G, et al. Consensus definition of sarcopenia, cachexia and pre-cachexia: joint document elaborated by Special Interest Groups (SIG) "cachexia-anorexia in chronic wasting diseases" and "nutrition in geriatrics". Clin Nutr. 2010;29(2):154-9.

33. Bijlsma AY, Meskers CG, Westendorp RG, Maier AB. Chronology of age-related disease definitions: osteoporosis and sarcopenia. Ageing Res Rev. 2012;11(2):320-4.

34. Anker SD, Morley JE, von Haehling S. Welcome to the ICD-10 code for sarcopenia. J Cachexia Sarcopenia Muscle. 2016;7(5):512-4.

35. Cao L, Morley JE. Sarcopenia Is Recognized as an Independent Condition by an International Classification of Disease, Tenth Revision, Clinical Modification (ICD-10CM) Code. J Am Med Dir Assoc. 2016;17(8):675-7.

36. Lechleitner M. [Obesity in elderly]. Wien Med Wochenschr. 2016;166(3-4):143-6.

37. Gariballa S, Alessa A. Sarcopenia: prevalence and prognostic significance in hospitalized patients. Clin Nutr. 2013;32(5):772-6.

38. Barbosa-Silva MC, Barros AJ, Wang J, Heymsfield SB, Pierson RN, Jr. Bioelectrical impedance analysis: population reference values for phase angle by age and sex. Am $\mathrm{J}$ Clin Nutr. 2005;82(1):49-52.

39. Genton L, Norman K, Spoerri A, Pichard C, Karsegard VL, Herrmann FR, et al. Bioimpedance-Derived Phase Angle and Mortality Among Older People. Rejuvenation Res. 2016. 
40. Bouillanne O, Dupont-Belmont C, Hay P, Hamon-Vilcot B, Cynober L, Aussel C. Fat mass protects hospitalized elderly persons against morbidity and mortality. Am J Clin Nutr. 2009;90(3):505-10.

41. Peel NM, Kuys SS, Klein K. Gait speed as a measure in geriatric assessment in clinical settings: a systematic review. J Gerontol A Biol Sci Med Sci. 2013;68(1):39-46.

42. Pamoukdjian F, Paillaud E, Zelek L, Laurent M, Levy V, Landre T, et al. Measurement of gait speed in older adults to identify complications associated with frailty: A systematic review. J Geriatr Oncol. 2015;6(6):484-96.

43. Liu WY, Meijer K, Delbressine JM, Willems PJ, Franssen FM, Wouters EF, et al. Reproducibility and Validity of the 6-Minute Walk Test Using the Gait Real-Time Analysis Interactive Lab in Patients with COPD and Healthy Elderly. PLoS One. 2016;11(9):e0162444.

44. Janssen I, Heymsfield SB, Baumgartner RN, Ross R. Estimation of skeletal muscle mass by bioelectrical impedance analysis. J Appl Physiol (1985). 2000;89(2):465-71.

45. Janssen I, Heymsfield SB, Ross R. Low relative skeletal muscle mass (sarcopenia) in older persons is associated with functional impairment and physical disability. J Am Geriatr Soc. 2002;50(5):889-96. 
Supplementary table 1: Case summary of the 3 most frequent co-morbid diseases according CIRS category of the acutely hospitalized geriatric patients $(n=81)$

\begin{tabular}{|c|c|c|c|c|c|c|c|}
\hline Cat. & Organ system & $1^{\text {st }}$ & $\mathrm{n}$ & $2^{\text {nd }}$ & $\mathrm{n}$ & $3^{\text {th }}$ & $\mathrm{n}$ \\
\hline 1 & Cardiac & Chronic heart failure & 33 & $\begin{array}{l}\text { Coronary artery } \\
\text { disease }\end{array}$ & 13 & Atrial Fibrillation & 12 \\
\hline 2 & Hypertension & 2 medications & 34 & 3 medications & 13 & 1 medication & 8 \\
\hline 3 & Vascular & Anaemia & 56 & $\begin{array}{l}\text { Peripheral vascular } \\
\text { disease }\end{array}$ & 11 & DVT/PE & 8 \\
\hline 4 & Respiratory & COPD & 34 & Pneumonia & 13 & Smoking & 11 \\
\hline 5 & ENT & Presbyacusis & 56 & Presbyopia & 24 & Cataract & 12 \\
\hline 6 & Upper GI & $\begin{array}{l}\text { Proton Pump } \\
\text { Inhibiter use }\end{array}$ & 25 & Reflux Esophagitis & 19 & Peptic Ulcer & 9 \\
\hline 7 & Lower GI & Constipation & 31 & Diverticular disease & 29 & Colon cancer & 4 \\
\hline 8 & Hepatic & Cholecystectomy & 13 & Cholecystolithiasis & 4 & Pancreatitis & 2 \\
\hline 9 & Renal & Renal failure & 19 & Stones & 10 & Renal Cell Carcinoma & 2 \\
\hline 10 & Other GU & Incontinence & 34 & Bladder retention & 14 & BPH/TURP & 11 \\
\hline 11 & $\begin{array}{l}\text { Musculoskeletal- } \\
\text { Integumentary }\end{array}$ & Osteoarthritis & 36 & Fracture & 18 & Rheumatic arthritis & 11 \\
\hline 12 & Neurological & Stroke/TIA & 34 & Parkinson(ism) & 5 & Epilepsy & 4 \\
\hline 13 & $\begin{array}{l}\text { Endocrine/ } \\
\text { metabolic }\end{array}$ & DM & 30 & Thyroid disease & 6 & Hypercholesterolemia & 11 \\
\hline 14 & $\begin{array}{l}\text { Psychiatric/ } \\
\text { Behavioural }\end{array}$ & Dementia & 39 & Delirium & 27 & $\mathrm{MCl}$ & 14 \\
\hline
\end{tabular}

CIRS: Cumulative IIIness Rating Scale; ENT: Ear, Nose, Throat; GI: Gastrointestinal; COPD: Chronic Obstructive Pulmonary Disease; TIA: Transient Ischaemic Attack; DM: Diabetes Mellitus; DVT: Deep Venous Thrombosis; PE: Pulmonary Embolism; BPH: Benign Prostatic Hypertrophy; TURP: Transurethral Resection of the Prostate; MCl: Mild Cognitive Impairment 
Supplementary table 2: Case summary of main medical diagnosis at hospital admission of the acutely hospitalized geriatric patients $(n=81)$

Main medical diagnosis

Number

Pneumonia

Delirium

Decompensated heart failure

Vertebral osteoporotic fracture

Falls/syncope

Septic shock

Cachexia

Anaemia

Medication intoxication

Diverticulitis

Erysipelas

Gastric cancer

Other*

${ }^{*}$ Acute cholecystitis, endocarditis, glomerulonephritis, gout arthritis, pulmonary embolism, myasthenia gravis, acute renal failure, osteomyelitis, polymyalgia rheumatic, SIADH, spondylodiscitis, subdural hematoma. All the geriatric patients had at least 5 other medical diagnosis/problems at hospital admission. 
Supplementary table 3: Case summary of sarcopenia according to EWGSOP, IWGS, SIG, FNIH consensus criteria in acutely hospitalized geriatric patients $(n=81)$ and 1-year mortality. 1-year

\begin{tabular}{|c|c|c|c|c|}
\hline & \multicolumn{2}{|c|}{ Non-sarcopenic } & \multicolumn{2}{|c|}{ Sarcopenic } \\
\hline & Alive & Dead & Alive & Dead \\
\hline EWGSOP & $33(83 \%)$ & $7(17 \%)$ & $17(41 \%)$ & $24(59 \%)$ \\
\hline IWGS & $17(77 \%)$ & $5(23 \%)$ & $33(56 \%)$ & $26(44 \%)$ \\
\hline SIG & $13(52 \%)$ & $12(48 \%)$ & $38(68 \%)$ & $18(32 \%)$ \\
\hline FNIH & $42(71 \%)$ & $17(29 \%)$ & $8(36 \%)$ & $14(64 \%)$ \\
\hline
\end{tabular}

Data represent the absolute number (and the \%) of patients who deceased and were alive after 1 year according to EWGSOP, IWGS, SIG, FNIH consensus criteria of sarcopenia.

EWGSOP=European Working Group on Sarcopenia in Older People; IWGS= International Working Group on Sarcopenia; SIG= Special Interest Group of Sarcopenia, Cachexia and Wasting Disorders; FNIH= Foundation for the National Institutes of Health 
Supplementary table 4: Cox proportional hazard ratio of 1-y mortality for acutely hospitalized geriatric patients with sarcopenia vs. no sarcopenia according the EWGSOP, IWGS, SIG and FNIH criteria for sarcopenia $(n=81)$.

\begin{tabular}{l|ccccc} 
& no sarcopenia & sarcopenia & HR & Cl -95\% & $\boldsymbol{P}$ \\
\hline EWGSOP & 40 & 41 & 4.273 & $1.838-10.000$ & $0.001^{*}$ \\
IWGS & 22 & 59 & 2.174 & $0.835-5.681$ & 0.112 \\
SIG & 25 & 56 & 0.529 & $0.259-1.082$ & 0.081 \\
FNIH & 59 & 22 & 2.778 & $1.362-5.650$ & $0.005^{*}$ \\
\hline
\end{tabular}

Data represent the Cox proportional hazard ratio of mortality in acutely hospitalized geriatric patients with sarcopenia compared with no sarcopenia after 1 year according to EWGSOP, IWGS, SIG, FNIH consensus criteria of sarcopenia.

EWGSOP=European Working Group on Sarcopenia in Older People; IWGS= International Working Group on Sarcopenia; SIG= Special Interest Group of Sarcopenia, Cachexia and Wasting Disorders; FNIH= Foundation for the National Institutes of Health

*significantly different hazard ratio between patients with or without sarcopenia 
Supplementary Table 5: Body composition, muscle strength physical function, frailty, nutrition, $A D L$, comorbidity and age versus 1-year survival in acutely hospitalized geriatric patients $(n=81)$.

$$
\text { Women }(n=59)
$$$$
\text { Men ( } n=22)
$$

\begin{tabular}{|c|c|c|c|c|c|c|c|c|}
\hline \multicolumn{9}{|c|}{ General } \\
\hline Age & $85.2 \pm 5.1$ & 22 & $84.4 \pm 5.6$ & 37 & $84.4 \pm 7.4$ & 9 & $81.6 \pm 6.2$ & 13 \\
\hline BMI & $22.9 \pm 4.9$ & 22 & $26.4 \pm 4.7^{*}$ & 37 & $23.2 \pm 4.2$ & 9 & $25.5 \pm 4.7^{*}$ & 13 \\
\hline \multicolumn{9}{|c|}{ Body composition } \\
\hline FFM, kg & $37.6 \pm 4.5^{\#}$ & 22 & $39.6 \pm 5.5^{\#}$ & 37 & $48.9 \pm 6.4$ & 9 & $51.2 \pm 9.3$ & 13 \\
\hline SMI, $\mathrm{kg} / \mathrm{m}^{2}$ & $6.2 \pm 0.5^{\#}$ & 22 & $6.6 \pm 0.6^{\star \# \#}$ & 37 & $7.6 \pm 1.0$ & 9 & $8.3 \pm 0.9^{*}$ & 13 \\
\hline RMM, \% & $27.9 \pm 4.2^{\#}$ & 22 & $25.6 \pm 3.1^{\#}$ & 37 & $34.2 \pm 2.1$ & 9 & $33.5 \pm 4.3$ & 13 \\
\hline Phase angle & $6.3 \pm 1.4$ & 22 & $6.7 \pm 1.4^{*}$ & 37 & $5.7 \pm 1.7$ & 9 & $7.4 \pm 1.6^{*}$ & 13 \\
\hline $\mathrm{BCM}, \mathbf{k g}$ & $19.5 \pm 2.0^{\#}$ & 22 & $20.4 \pm 2.5^{\star \#}$ & 37 & $24.7 \pm 2.4$ & 9 & $27.0 \pm 3.9^{*}$ & 13 \\
\hline FMI, $\mathrm{kg} / \mathrm{m}^{2}$ & $7.8 \pm 4.2^{\#}$ & 22 & $10.7 \pm 4.1^{\star \#}$ & 37 & $5.3 \pm 1.7$ & 9 & $7.7 \pm 3.2^{*}$ & 13 \\
\hline FM\% & $33.0 \pm 9.3^{\#}$ & 22 & $39.4 \pm 8.3^{\star \#}$ & 37 & $23.3 \pm 5.1$ & 9 & $30.1 \pm 7.3^{*}$ & 13 \\
\hline SMM/FM & $1.0 \pm 0.5^{\#}$ & 22 & $0.7 \pm 0.3^{\star \#}$ & 37 & $1.5 \pm 0.3$ & 9 & $1.2 \pm 0.5^{*}$ & 13 \\
\hline
\end{tabular}

\section{Physical function}

\begin{tabular}{lllllllll}
\hline HGS Jamar, kg & $15.6 \pm 5.3^{\#}$ & 22 & $15.6 \pm 5.9^{\#}$ & 37 & $23.5 \pm 5.7$ & 9 & $26.8 \pm 7.5$ & 13 \\
GS, $\mathbf{m} / \mathbf{s}$ & $0.38 \pm 0.13^{\#}$ & 12 & $0.49 \pm 0.2^{\star \#}$ & 27 & $0.46 \pm 0.2$ & 9 & $0.74 \pm 0.4^{*}$ & 13 \\
SPPB & $2.1 \pm 2.0^{\#}$ & 22 & $3.0 \pm 2.5^{\#}$ & 37 & $4.2 \pm 2.4$ & 9 & $4.4 \pm 3.3$ & 13 \\
HABAM & $34.6 \pm 13.1$ & 22 & $36.0 \pm 14.4$ & 37 & $37.6 \pm 9.2$ & 9 & $44.5 \pm 14.6$ & 13
\end{tabular}

Frailty, nutrition, ADL, comorbidity and cognitive function

\begin{tabular}{|c|c|c|c|c|c|c|c|c|}
\hline Fried score & $4.1 \pm 0.7$ & 22 & $3.9 \pm 0.6^{*}$ & 37 & $4.1 \pm 0.6$ & 9 & $3.5 \pm 0.8^{*}$ & 13 \\
\hline GFI & $8.4 \pm 2.5$ & 22 & $7.3 \pm 2.7$ & 35 & $7.7 \pm 2.5$ & 9 & $7.3 \pm 2.7$ & 13 \\
\hline SNAQ & $2.3 \pm 1.7$ & 22 & $1.6 \pm 1.6$ & 35 & $1.9 \pm 1.7$ & 9 & $2.1 \pm 1.4$ & 13 \\
\hline Katz-ADL & $3.5 \pm 2.1$ & 22 & $3.6 \pm 2.2$ & 35 & $3.1 \pm 2.4$ & 9 & $4.1 \pm 1.5$ & 13 \\
\hline CIRS & $19.2 \pm 4.4$ & 22 & $19.5 \pm 5.6$ & 37 & $22.9 \pm 4.2$ & 9 & $19.8 \pm 6.6$ & 13 \\
\hline MMSE & $19.4 \pm 5.4$ & 18 & $19.9 \pm 5.8$ & 29 & $19.2 \pm 5.0$ & 9 & $20.1 \pm 5.4$ & 13 \\
\hline \multicolumn{9}{|c|}{$\begin{array}{l}\text { Data are means } \pm \text { SD. BMI: Body Mass Index; FFM: Fat Free Mass; SMI: Skeletal Muscle Mass Index; RRM: Relative } \\
\text { Muscle Mass; BCM: Body Cell Mass; FMI: Fat Mass Index; FM\%: Fat Mass Percentage; SMM/FM: Skeletal Muscle } \\
\text { Mass/Fat Mass; HGS Jamar: Handgrip Strength measured with Jamar dynamometer; GS: Gait Speed; SPPB: Short } \\
\text { Physical Performance Battery; HABAM: Hierarchical Balance and Mobility; GFI: Groningen Frailty Indicator; SNAQ: } \\
\text { Short Nutritional Assessment Questionnaire; CIRS: Cumulative Illness Rating Scale; MMSE: Minimal Mental State } \\
\text { Examination; significantly different from deceased (P<.05); " significantly different from men }(P<.05) \text {. }\end{array}$} \\
\hline
\end{tabular}


Supplementary table 6: Hazard Ratios for potential predictors for 1-y mortality in hospitalized geriatric patients $(n=81)$ with additional analysis including gait speed $(n=61)$

1-y mortality probability

\begin{tabular}{l|clccc}
\hline & $\mathrm{n}$ & & HR & Cl-95\% & $P$ \\
\hline Step 1 & 81 & FMI & 0.835 & $0.743-0.939$ & $0.003^{*}$ \\
Step 2 & & PA & 0.706 & $0.536-0.931$ & $0.013^{*}$ \\
& & FMI & 0.835 & $0.743-0.940$ & $0.003^{*}$ \\
\hline Step 1 & 61 & FMI & 0.783 & $0.666-0.920$ & $0.003^{*}$ \\
Step 2 & & FMI & 0.764 & $0.651-0.897$ & $0.001^{*}$ \\
& & GS & 0.030 & $0.002-0.535$ & $0.017^{*}$
\end{tabular}

Data represent the results of Cox proportional hazard analysis for FMI, PA and SMI for 1-year mortality in acutely hospitalized geriatric patients $(n=81)$. Additionally Cox proportional hazard mortality analysis were performed for the patients with available gait speed at hospital admission (n=61) FMI: Fat Mass Index; PA: Phase Angle; GS: Gait Speed. *significant $(P<.05)$ 


\title{
CHAPTER 4
}

\section{Multi-frequency bioelectrical impedance}

\author{
analysis may represent a reproducible and
}

practical tool to assess skeletal muscle

\section{mass in euvolemic acutely ill hospitalized}

\author{
geriatric patients
}

Keywords: bio-impedance, sarcopenia, hydration, aging, hospitalization

Walther MWH Sipers, Jesper Dorge, Jos MGA Schols,

Lex B Verdijk, Luc JC van Loon

European Geriatric Medicine (2020) 11, 155-162

doi: 10.1007/s41999-019-00253-6 


\begin{abstract}
Purpose: Geriatric patients with low skeletal muscle mass (SMM) and strength have a poor clinical outcome following acute illness. Consequently, it is recommended to assess SMM and strength in patients admitted to the acute care geriatric ward. Bio-impedance analysis $(\mathrm{BIA})$ is a practical tool to assess SMM in hospitalized patients. However, the reproducibility of this assessment may be compromised due to changing clinical conditions. The objective was to study the reproducibility of SMM assessment using multi frequency BIA (mf-BIA) in acutely ill geriatric patients.
\end{abstract}

Methods: A total of 47 geriatric patients (age: $83 \pm 7 \mathrm{y} ; n=31$ female) admitted to the acute geriatric ward participated in this pilot study. SMM was assessed on three occasions within the first week of hospital admission using the Maltron Bioscan-920-II.

Results: Total skeletal SMM averaged $21.4 \pm 5.7,20.7 \pm 5.4$, and $20.8 \pm 5.1 \mathrm{~kg}$ assessed at $2 \pm 1$, $3 \pm 1$ and $5 \pm 2$ days after hospital admission, respectively. Coefficient of variation (COV) of the three SMM measurements was $4.9 \pm 4.5 \%$ with an Intraclass Correlation Coefficient (ICC) of $0.976(\mathrm{Cl}-95 \%$ : 0.961-0.986; $P<0.001)$. Hydration status affected the reproducibility of the measurement, with non-euvolemic patients $(n=16)$ showing a significantly higher COV $(7.6 \pm 5.9 \%$ vs $3.5 \pm 2.9 \% ; P<0.01)$ and a lower ICC $(0.983$ vs $0.913 ; P<0.001)$ when compared to the euvolemic patients $(n=31)$.

Conclusion: Mf-BIA seems a highly reproducible and reliable method to assess SMM throughout the first week of hospitalization in geriatric patients. However, since abnormal hydration status may compromise reliability of the measurement, assessment of SMM using mf-BIA may better be performed when euvolemic status has been established. 


\section{Introduction}

Skeletal muscle mass and strength are strong prognostic factors for the functional decline, morbidity, and mortality of older patients (1-4). Low skeletal muscle mass and poor physical performance are highly prevalent in hospitalized geriatric patients (5-8). Clinical outcome following acute illness is generally poor in these geriatric patients with low skeletal muscle mass and strength $(5,9-11)$. Consequently, it is recommended to assess skeletal muscle mass and strength in patients admitted to the acute care geriatric ward $(12,13)$.

Bio-impedance analysis (BIA) is the prefered method to measure skeletal muscle mass in the acute care geriatric ward (13-15). This is because the geriatric patient is clinically compromised and the BIA application can be done at bedside, is non-invasive, imposes no radiation exposure, and the measurement is relatively inexpensive and readily available in many hospitals $(14,16)$.

BIA was introduced in the 1950s to measure body composition and is based on the principle that tissues rich in water and electrolytes, e.g. the skeletal muscle mass, are less resistant to an electrical current than lipid-rich tissue $(14,17-21)$. The many available BIA systems range from single to multiple frequency, employ contact or gel electrodes, and measure whole-body or segmental (upper-lower arm, upper-lower leg, and the trunk) electrical pathways (19-22). All BIA systems measure impedance and resistance (caused by the total water across the body) and reactance (due to capacitance of cell membranes). These measurements are incorporated into automated body composition prediction equations that are population specific, usually taking into account age, gender, ethnicity, height, and weight $(21,23,24)$.

To accurately assess skeletal muscle mass with BIA, it is required that the method is reproducible. To our knowledge there is no study evaluating the reproducibility of skeletal muscle mass assessment with mf-BIA in acutely ill hospitalized geriatric patients. Reproducibility of skeletal muscle mass assessment with BIA could be severely compromised in this population for several reasons. Acute care geriatric ward patients frequently experience issues with hydration status upon hospital admission, and hydration status can subsequently change dramatically during the first week of hospitalization due to strategies to resolve hydration problems, malnutrition, progression of disease, treatment and/or recovery from acute illness. Therefore, accurate assessment of muscle mass in the geriatric patient using BIA may be compromised when applied throughout the first week of hospital admission. In the present pilot study, we therefore aimed to assess the reproducibility of skeletal muscle mass assessment using BIA in acutely hospitalized geriatric patients. Muscle mass was assessed within 2 days after hospital admission and on 2 more occasions throughout the first week of hospitalization in the acute care geriatric ward. 


\section{Methods}

Study sample

All geriatric patients admitted to the acute geriatric ward of a Dutch general hospital were asked to participate in the study. In the 5 month period of recruitment, from October 2014 through February 2015, we intended to include as many patients as possible. The inclusion criteria were: age above 70 y and being frail according to the Fried criteria $(25,26)$. Patients were excluded if they had a terminal condition (avoiding unnecessary burden for those expected to die within 2-3 weeks), an implantable cardioverter defibrillator (ICD) or if no informed consent was obtained from the patient or proxy.

\section{Patient characteristics}

Patient characteristics were retrieved from the medical and nursing files. These included sex, age, diagnosis at hospital admission, medical history, fluid management during hospitalization (e.g. diuretics or intravenous fluid), C-reactive protein (CRP) as a marker of inflammation, nutritional status, and the acute illness that led to hospital admission. Since C-reactive protein (CRP) levels higher than $100 \mathrm{mg} / \mathrm{L}$ are severely elevated and almost always a sign of severe bacterial infection, patients were classified as either higher or lower than $100 \mathrm{mg} / \mathrm{L}$ (27). Height was estimated to the nearest $\mathrm{cm}$ by measuring ulna length because many patients were temporarily bedridden (28). The frailty score was assessed according to the Fried criteria, which ranges from 0 to 5 : a score of 3 or higher indicates frailty $(25,26)$. The fifth item of the Fried criteria "low physical activity" is based on the question (obtained from patient or caregiver): are you at least 5 days a week during 30 minutes a day physically active like walking or biking? Test is positive if this physical activity is not achieved (25). Body weight was measured on a sitting weight scale (SECA, Model 959).

A number of scales was used to evaluate patient status. The Katz ADL-6 was used as a validated instrument for screening daily living activities (ADL) (29). Scores range from 0 (totally independent) to 6 (completely dependent) (29). The cumulative illness rating scale (CIRS) was used to calculate the number and severity of chronic illnesses of the patients' comorbid diseases. The score ranges from 0 , which corresponds to the absence of disorders, to a maximum of 56 (30). Malnutrition was measured using the Short Nutritional Assessment Questionnaire (SNAQ), which is a validated screening instrument for malnutrition. Scores range from 0 to 5 ; a score of 3 or higher indicates that the patient is malnourished (31).

\section{Body composition measurement}

The Maltron BioScan 920-II, a multi-frequency bioelectrical impedance analysis (mf-BIA) device, was used to measure skeletal muscle mass (SMM). The Maltron Bioscan 920-II has been validated for the assessment of body composition and muscle mass at the whole-body 
level as well as segmental muscle mass in healthy older people $(32,33)$. The Maltron BioScan 920-II has an eight-point electrode system, which separately measures impedance of the patient's trunk, arms and legs at four different frequencies $(5 \mathrm{kHz}, 50 \mathrm{kHz}, 100 \mathrm{kHz}$ and 200 $\mathrm{kHz}$ ) for each body segment. The Maltron BioScan 920-II calculates SMM according to the device-specific calculation called the Maltron calculation (33). BIA was performed with patients only wearing their pyjamas, and electrodes were placed on foot, ankle, knee, hip, hand, wrist, elbow and shoulder (8) (http://www.maltronint.com/). Three repeated measurements were performed for each patient, all on the same moment of the day (either in the morning or in the afternoon); within 2 days after hospital admission, and on 2 more occasions throughout the first week of hospitalization in the acute care geriatric ward, and at least one day apart. Since we aimed to assess reliability of the SMM assessment per se, and we were unable to accurately assess height in bedridden patients, only true SMM values are presented, rather than 'adjusted' values such as SMM Index (SMMI).

\section{Clinical judgement of hydration status}

No single 'gold-standard' marker of hydration status exists $(34,35)$. In clinical practice, parameters like skin turgor, axillary dryness, dry mucous membranes in combination with blood biochemistry including plasma osmolality, electrolytes, and blood urea nitrogen to creatinine ratio $(\mathrm{BUN}: \mathrm{Cr})$ represent a criterion method of identifying dehydration or overhydration (36). In our study the assessment of hydration status (normal, dehydrated or overhydrated) was based on signs and symptoms of dehydration (poor skin turgor, axillary dryness, dry mucous membranes of mouth and tongue or orthostatic blood pressure) or signs and symptoms of overhydration (presence of oedema, signs of decompensated heart failure, including chest $\mathrm{x}$-ray, if present) in combination with $\mathrm{BUN}: \mathrm{Cr}$ ratio and potassium level. This clinical judgement of hydration status was done by an experienced ward resident at hospital admission and during the daily rounds during the first week of hospital stay.

\section{Statistics}

Data were analysed using SPSS Statistics version 23 (IBM Corp., Armonk, NY, USA). Due to the practical/clinical setting of this study, we intended to include as many patients as possible throughout the 5-month study period, without performing pre-set sample size calculations. Patients' characteristics are described by mean $\pm S D$ and range for continuous variables (after checking for normality) and by frequencies and percentages for the categorical variables. The variability between the different measurements of the absolute skeletal muscle mass (SMM) was determined by calculating the coefficient of variation (COV). Repeated measures ANOVA test was used to determine systematic differences in SMM between the three occasions of 
measurement, since SMM was normally distributed based on Kolmogorov-Smirnov testing. To determine test-retest reliability between the 3 measurements during the first week of hospitalization, Intraclass Correlation Coefficients (ICC) for absolute agreement and average measures were calculated, including $95 \%$ confidence intervals $(95 \% \mathrm{Cl})$.

To determine the influence of the hydration status (based on clinical judgment) and time effect on repeated BIA measurement of SMM, an additional repeated measures ANOVA was performed with hydration status added as group factor. Furthermore, Mann Whitney-U test was performed to compare COV values between patients with a normal hydration status and patients categorized as dehydrated or overhydrated. Moreover, ICC and $95 \% \mathrm{Cl}$ values were calculated for euvolemic and non-euvolemic patients separately.

Finally, repeated measures ANOVA was also performed to study the influence of presence of fluid management (applying intravenous fluid or diuretics vs. no fluid correction) and presence of low or high inflammation (low systemic inflammation with CRP $<100 \mathrm{mg} / \mathrm{L}$ vs. high systemic inflammation with CRP> 99mg/L) on mf-BIA measurement of SMM during hospitalization. Furthermore, COV and ICC values were also calculated and compared between these groups.

\section{Results}

Patients

In a four-month period, 122 older patients were admitted to the acute geriatric ward and asked to participate. Thirteen patients or proxies refused to participate and another 26 were excluded because primary admission was on a different hospital ward. Of the remaining 83 patients, 36 were excluded from the present analysis, because of missing one or more BIA measurements due to early hospital discharge. Mean $\pm S D$ age of the remaining 47 patients was $83 \pm 7$ y and $66 \%(n=31)$ were female. All patients were frail with a mean Fried score of $4.1 \pm 0.7$. Forty-two percent of the patients were malnourished, with SNAQ scores of 3 or higher. Forty-two percent were highly ADL dependent, with a Katz ADL- 6 score of 5 or 6 . The mean CIRS score was

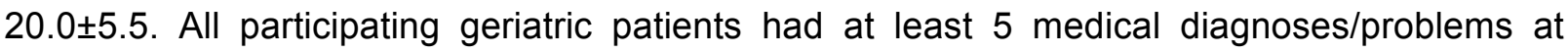
hospital admission. The most frequent diagnoses at hospital admission were pneumonia $(n=21)$, delirium $(n=15)$, dehydration $(n=11)$, urinary tract infection $(n=8)$, anaemia $(n=7)$, vertebral fracture $(n=6)$, decompensated heart failure $(n=5)$, falls $(n=6)$, medication intoxication $(n=4)$, septic shock $(n=2)$, and others $(n=12)$. The first mf-BIA measurement was performed at $2 \pm 1$ days after hospital admission and the second and third measurements were performed at respectively $3 \pm 1$ and $5 \pm 2$ days after hospital admission. Based on clinical judgement, $n=31$ patients were euvolemic and $n=16$ patients were either over- or dehydrated. See table 1 for a summary of patients' characteristics separated for hydration status. CRP was lower and handgrip strength was greater in euvolemic vs non-euvolemic patients. 
Table 1: Patients' characteristics ( $n=47)$ categorized based on clinical judgement of hydration status as euvolemic $(n=31)$ and abnormal $(n=16)$

\begin{tabular}{lcc}
\hline & Euvolemic Hydration Status & Abnormal Hydration status \\
\hline Number of patients & 31 & 16 \\
Age $(\mathrm{y})$ & $83.4 \pm 5.5$ & $83.7 \pm 8.6$ \\
Female $(\mathrm{n}, \%)$ & $19(61)$ & $4(25)$ \\
Weight $(\mathrm{kg})$ & $74.2 \pm 14.4$ & $64.8 \pm 18.5$ \\
CRP $(\mathrm{mg} / \mathrm{L})$ & $67.6 \pm 65.6$ & $136.4 \pm 110.8^{*}$ \\
HGS Jamar $(\mathrm{kg})$ & $15.7 \pm 8.0$ & $10.8 \pm 6.5^{*}$ \\
Fried score & $4.0 \pm 0.7$ & $4.3 \pm 0.7$ \\
SNAQ & $2.2 \pm 1.8$ & $2.4 \pm 1.9$ \\
Katz ADL-6 & $3.8 \pm 2.0$ & $3.4 \pm 2.9$ \\
\hline
\end{tabular}

CRP: C-reactive protein; HGS Jamar: Handgrip Strength measured with Jamar dynamometer; SNAQ: Short Nutritional Assessment Questionnaire; Katz ADL-6: activities of daily living

*Significantly different from euvolemic patients $(P<.05)$

\section{Skeletal muscle mass}

Mean absolute SMM was $21.4 \pm 5.7 \mathrm{~kg}$ at the first, $20.7 \pm 5.4 \mathrm{~kg}$ at the second, and $20.8 \pm 5.1 \mathrm{~kg}$ at the third measurement. No systematic differences in mean SMM were observed between the first, second and third day of measurement with mf-BIA during the first week after hospital admission $(P=0.129)$. The mean COV calculated over the three mf-BIA measurements of SMM was $4.9 \pm 4.5 \%$. Similar findings were observed for the first and second measurement, the first and third measurement and the second and third measurement separately (Table 2).

Table 2: Covariance of repeated mf-BIA measurement of Skeletal Muscle Mass on three different days in the first week after hospitalization in acutely ill geriatric patients $(n=47)$.

\begin{tabular}{l|llll}
\hline Time point & $\mathbf{1 - 2}$ & $\mathbf{1 - 3}$ & $\mathbf{2 - 3}$ & $\mathbf{1 - 2 - 3}$ \\
\hline Mean SMM, kg & 21.1 & 21.1 & 20.8 & $\mathbf{2 1 . 0}$ \\
SD SMM & 5.5 & 5.3 & 5.2 & $\mathbf{5 . 3}$ \\
Mean COV, \% & 4.8 & 4.4 & 3.8 & $\mathbf{4 . 9}$ \\
SD cov & 5.2 & 5.2 & 4.5 & $\mathbf{4 . 5}$ \\
\hline
\end{tabular}

mf-BIA: multifrequency bio-impedance analysis; SMM: absolute skeletal muscle mass; COV: coefficient of variation; $S D$ : standard deviation

Test-retest reliability for the three mf-BIA measurements of SMM was shown to be high with an ICC of 0.976 (95\% Cl: 0.961-0.986; $P<0.001)$. Also for ICC, similar findings were observed when separate analyses were performed for the first and second measurement, the first and third measurement and the second and third measurement (Table 3). In 10 patients all the BIA measurements took place in the afternoon and the results for COV and ICC did not differ from 
the patients measured early in the morning (data not shown).

Table 3: Intraclass Correlation Coefficients (ICC) of repeated mf-BIA measurement of SMM on three different days in the first week after hospitalizaton in acutely ill geriatric patients $(n=47)$.

\begin{tabular}{cccc}
\hline Time point & ICC $^{*}$ & $\mathbf{9 5 \% C l}$ & P value \\
\hline $\mathbf{1 - 2}$ & 0.960 & $0.925-0.978$ & $<0.001$ \\
$\mathbf{2 - 3}$ & 0.976 & $0.957-0.987$ & $<0.001$ \\
$\mathbf{1 - 3}$ & 0.959 & $0.926-0.977$ & $<0.001$ \\
$\mathbf{1 - 2 - 3}$ & $\mathbf{0 . 9 7 6}$ & $\mathbf{0 . 9 6 1 - 0 . 9 8 6}$ & $<0.001$ \\
\hline
\end{tabular}

mf-BIA: multifrequency bio-impedance analysis; SMM: absolute skeletal muscle mass

*Two-mixed model with absolute agreement definition

Clinical hydration status and skeletal muscle mass

According to clinical judgement at the first moment of mf-BIA measurement, $n=31$ (66\%), $n=11$ $(23 \%)$ and $n=5(11 \%)$ patients were categorized as euvolemic, dehydrated and overhydrated, respectively. These numbers changed to $n=28(60 \%), n=11(23 \%)$, and $n=8(17 \%)$ at the second assessment, and $n=39(83 \%), n=3(6 \%)$, and $n=5(11 \%)$ at the third assessment, respectively. No differences in mean SMM measured with mf-BIA were observed in patients with normal or abnormal hydration status between the first, second and third day of measurement (Table 4).

For euvolemic patients separately (at first measurement, $n=31$ ), the mean COV of SMM calculated over all the three time points of mf-BIA measurement was $3.5 \pm 2.9 \%$ (Table 4 , Figure 1).

Table 4: Covariance of repeated mf-BIA measurement of SMM on three different days in the first week after hospitalization in acutely ill geriatric patients categorized as euvolemic at the first measurement day $(n=31)$, euvolemic on all three measurement days $(n=21)$ and dehydrated or overhydrated at the first measurement day $(n=16)$.

\begin{tabular}{|c|c|c|c|c|c|c|c|c|c|c|c|c|}
\hline \multirow[b]{2}{*}{ Time point } & \multicolumn{4}{|c|}{ Initial euvolemic $(n=31)$} & \multicolumn{4}{|c|}{$\begin{array}{l}\text { Euvolemic at } 3 \text { time points } \\
\qquad(n=21)\end{array}$} & \multicolumn{4}{|c|}{$\begin{array}{l}\text { Initial dehydrated or } \\
\text { overhydrated }(n=16)\end{array}$} \\
\hline & $1-2$ & $1-3$ & $2-3$ & $1-2-3$ & $1-2$ & $1-3$ & $2-3$ & $1-2-3$ & $1-2$ & $1-3$ & $2-3$ & $1-2-3$ \\
\hline Mean SMM, kg & 21.7 & 21.6 & 21.6 & 21.6 & 21.6 & 21.1 & 21.1 & 21.3 & 19.9 & 20.1 & 19.3 & 19.7 \\
\hline SD SMM & 5.5 & 5.4 & 5.3 & 5.4 & 5.9 & 5.7 & 5.5 & 5.7 & 5.3 & 5.1 & 4.7 & 5.0 \\
\hline Mean COV, \% & 3.3 & 3.1 & 3.2 & 3.5 & 3.4 & 3.4 & 3.0 & 3.4 & 7.7 & 6.8 & 5.0 & 7.6 \\
\hline SD COV & 3.0 & 3.4 & 3.2 & 2.9 & 3.1 & 3.5 & 3.1 & 2.8 & 7.2 & 7.1 & 6.3 & 5.9 \\
\hline
\end{tabular}

mf-BIA: multifrequency bio-impedance analysis; SMM: absolute skeletal muscle mass; COV: coefficient of variation; $S D$ : standard deviation 


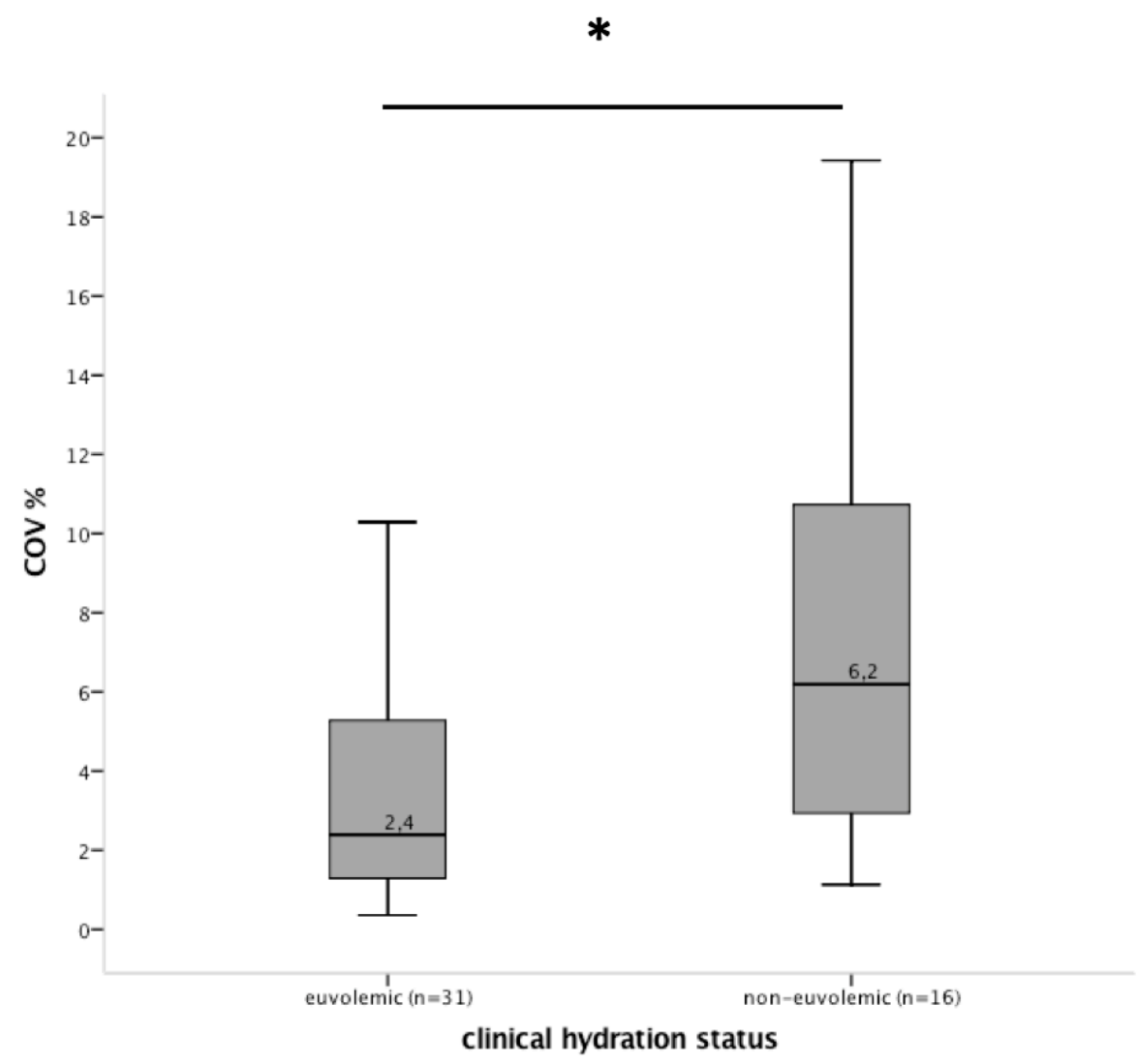

Figure 1: Coefficient of variation (COV) repeated mf-BIA measurement of Skeletal Muscle Mass on three different days during the first week after hospitalization in the acutely ill geriatric patients categorized as euvolemic $(n=31)$ and dehydrated or overhydrated $(n=16)$ at first measurement. Horizontal lines indicate median, boxes indicate 25 and 75 percentile and bars indicate minimal and maximal values. *: significant difference based on Mann-Whitney U-test ( $P=0.003)$

Similar findings were observed for the first and second measurement, the first and third measurement and the second and third measurement separately (Table 4). For patients that were euvolemic on all three occasions of BIA measurement $(n=21)$, the mean COV of SMM remained unchanged (Table 4). The COV's of mf-BIA measurement of SMM in patients with abnormal hydration status ( $n=16$; dehydrated or overhydrated) were significantly greater than those calculated for patients with euvolemic hydration status $(P=0.003)$. Mean COV of SMM for patients with abnormal hydration status calculated over all three time points was $7.6 \pm 5.9 \%$. Again, similar findings were observed for the first and second measurement, the first and third measurement, and the second and third measurement separately (Table 4).

In accordance with the COV data, test-retest reliability for repeated mf-BIA measurement of SMM was better in the euvolemic patients (mean ICC $0.983 ; 95 \% \mathrm{Cl}: 0.964-0.992 ; P<0.001$ ) compared to non-euvolemic patients (mean ICC $0.913 ; 95 \% \mathrm{Cl}: 0.711-0.971 ; P<0.001$ ). Since the point-estimate of ICC for euvolemic patients was not included in the $95 \% \mathrm{Cl}$ of the noneuvolemic patients (and vice versa), this difference was significant. 
Fluid management, inflammation and skeletal muscle mass

Seventy-two percent of the patients received intravenous fluid or loop diuretics prior to the first mf-BIA assessment. Prior to the second and third measurements, this was substantially less (34\% and $39 \%$, respectively). Mean SMM measured with mf-BIA did not change significantly over the three different days in the patients who did $(n=34)$ or did not receive fluid management prior the first measurement $(n=13)$. Upon the first mf-BIA measurement, mean CRP was $91 \pm 82 \mathrm{mg} / \mathrm{L}$ (range $1-377 \mathrm{mg} / \mathrm{L}$ ) with a median CRP value of $58 \mathrm{mg} / \mathrm{L}$. A plasma $\mathrm{CRP}<100 \mathrm{mg} / \mathrm{L}$ was categorized as a low level of systemic inflammation ( $n=31)$, a CRP $>99$ $\mathrm{mg} / \mathrm{L}$ was categorized as a high level of systemic inflammation $(n=16)$. No changes in SMM were observed over the three measurement days for both these groups.

No differences were observed for SMM, COV, or ICC of repeated SMM assessment between patients with or without intravenous fluid therapy or loop diuretics, nor between patients with a high level of systemic inflammation compared to those with a low level of systemic inflammation.

\section{Discussion}

In the present pilot study, we observed an overall reproducibility (COV) of $4.9 \%$ and test-retest reliability (ICC) of 0.976 for three repeated measurements of SMM using mf-BIA in the first week after hospitalization in acutely ill geriatric patients. For patients classified as having a normal hydration status based on clinical judgement, repeated mf-BIA measurement of SMM showed lower variation and, thus, better reproducibility compared to the patients with an abnormal hydration status.

Low SMM and strength in geriatric patients admitted to the acute care geriatric ward is of prognostic significance $(10,11,37)$. Therefore, measuring SMM is relevant in the acute care geriatric ward. BIA represents a relatively cheap, non-invasive, and easily accessible tool to assess SMM in these patients. However, there is little information about the reproducibility of the assessment of SMM using BIA in this patient group. We assessed SMM using mf-BIA in geriatric patients within 2 days of admission at the geriatric ward, with repeated measurements performed 1 and 3 days later. No systematic changes in SMM were observed over the three measurements. Previous work in healthy, older individuals has reported very good reproducibility of repeated BIA measurements, with a COV of $1.8 \%$ for muscle mass assessment ( $n=24,61 \pm 4$ y) (38). To our knowledge there are no studies addressing the reproducibility of repeated mf-BIA measurements of SMM in acutely ill hospitalized geriatric patients. Our findings show a COV of $4.9 \%$ for mf-BIA based muscle mass assessments calculated for three repeated measurements within the first week of hospitalization in acutely ill geriatric patients. Despite acceptable reproducibility (i.e., COV $<5 \%$ ), the COV for repeated 
measurements tends to be higher in this geriatric patient group when compared with healthy (older) individuals. Indeed, obvious differences between study populations likely affect the testretest reliability of the BIA measurement. We recruited acutely ill hospitalized geriatric patients with a high mean age of $83 \mathrm{y}$, a variety of diseases and co-morbidity, and measurements over a time period of changing (clinical) conditions and a variety of induced medical therapies like applying antibiotics, steroids, opioids, antipsychotics, nutritional support, bladder catheters etc. For example, in patients admitted to the acute geriatric ward, hydration status is often hampered. Indeed, $34 \%$ of the patients were not normally hydrated, with dehydration present in 11 patients and overhydration present in 5 patients. When separately calculating the reproducibility in both the dehydrated or overhydrated patients $(n=16)$, we observed a substantially higher COV of $7.6 \%$ compared to a COV of $3.5 \%$ in patients with a normal hydration status $(n=31)$. In accordance, the ICC for three repeated measurements was very high for the entire patient group (ICC: 0.976), but hydration status strongly affected the reliability. A much higher ICC and, thus, better reproducibility was observed for the patients with normal (ICC: 0.983) when compared with abnormal hydration status (ICC: 0.913). Notably, a COV of $7.6 \%$ could translate into a $\sim 1.6 \mathrm{~kg}$ error margin for SMM assessment in this population of geriatric patients, which we propose is unsatisfactory in terms of reliable SMM assessment. Although it may seem contradictory to the findings on hydration status, we observed no differences for reliability between patients that did or did not receive intravenous fluid or loop diuretics. This is likely explained by the fact that in clinical practice, some euvolemic patients actually receive (chronic) loop diuretics to prevent exacerbation of their heart failure, whereas some non-euvolemic patients did not receive intravenous fluid administration because they were able to drink and compensate for dehydration themselves. Altogether, our findings clearly underline that caution should primarily be taken when using $\mathrm{BIA}$ to assess skeletal muscle mass in patients with abnormal hydration status.

In accordance with clinical practice, assessment of hydration status in the present study was based on clinical judgment, taking both physical and laboratory parameters into account. Categorizing hydration status based on clinical judgement was partly subjective and could be a limitation of our study; however, there is no gold standard for assessing hydration status and judgement was performed by experienced clinicians in the current study. The first measurement of SMM with BIA took place between day one and day three of hospital stay, a period during which changes in hydration status can take place and may impact reproducibility of the measurements. However, no differences in mean SMM measured with mf-BIA were observed in patients with normal or abnormal hydration status between the first, second, and third measurement. Because measurement of SMM with BIA was in some patients $(n=10)$ performed in the afternoon and not in the early morning influence of food and drinks could have impacted reproducibility of the measurement. However, measurements were always 
performed at the same time of day for each individual patient, and no differences in mean COV and ICC of SMM measurement with mf-BIA were observed between patients with measurements performed in the early morning versus afternoon. As a final limitation, in this pilot study the number of non-euvolemic patients $(n=16)$ was relatively low. Although we were able to detect relevant differences between euvolemic and non-euvolemic patients, our findings need to be confirmed in additional studies with larger study samples. As with the current work, it would be preferred to do that within a clinical environment. As such, if the results of this study can be confirmed in larger studies it provides easily translatable, relevant clinical insight and provides clinicians information on the usefulness of mf-BIA measurement as well as with its limitations due to the influence of the patients' hydration status. Indeed, the strength of the present study is that it was performed in a real life setting in an acute care geriatric ward with the objective of studying the reproducibility of SMM with mf-BIA during hospitalization.

In this study, we show that bio-impedance measurement of SMM seems a practical, reproducible method with a high test-retest reliability in the geriatric patient admitted to the acute care geriatric ward. However, when patients are dehydrated or overhydrated, reliability of bio-impedance measurements may be compromised. Therefore, we propose that muscle mass assessment during the first week after hospitalization may better take place when the patient is clinically euvolemic. For non-euvolemic patients, we propose that it is likely better to perform mf-BIA based muscle mass assessment after correction of dehydration or overhydration through regular fluid management.

In conclusion, multiple frequency bio-impedance analysis seems a highly reproducible and reliable method to assess skeletal muscle mass throughout the first week of hospitalization in patients admitted to the acute care geriatric ward. However, since abnormal hydration status may compromise reliability of the measurement, assessment of muscle mass using bioimpedance may better be performed when euvolemic status has been established. 


\section{References}

1. Alexandre Tda S, Duarte YA, Santos JL, Wong R, Lebrao ML. Sarcopenia according to the European Working Group on Sarcopenia in Older People (EWGSOP) versus dynapenia as a risk factor for mortality in the elderly. J Nutr Health Aging. 2014;18(8):751-6.

2. Giampaoli S, Ferrucci L, Cecchi F, Lo Noce C, Poce A, Dima F, et al. Hand-grip strength predicts incident disability in non-disabled older men. Age Ageing. 1999;28(3):283-8.

3. Rantanen T, Avlund K, Suominen H, Schroll M, Frandin K, Pertti E. Muscle strength as a predictor of onset of ADL dependence in people aged 75 years. Aging Clin Exp Res. 2002;14(3 Suppl):10-5.

4. Metter EJ, Talbot LA, Schrager M, Conwit R. Skeletal muscle strength as a predictor of all-cause mortality in healthy men. J Gerontol A Biol Sci Med Sci. 2002;57(10):B35965.

5. Cerri AP, Bellelli G, Mazzone A, Pittella F, Landi F, Zambon A, et al. Sarcopenia and malnutrition in acutely ill hospitalized elderly: Prevalence and outcomes. Clin Nutr. 2015;34(4):745-51.

6. Sousa AS, Guerra RS, Fonseca I, Pichel F, Amaral TF. Sarcopenia among hospitalized patients - A cross-sectional study. Clin Nutr. 2015;34(6):1239-44.

7. Smoliner C, Sieber CC, Wirth R. Prevalence of sarcopenia in geriatric hospitalized patients. J Am Med Dir Assoc. 2014;15(4):267-72.

8. Sipers WM, Meijers JM, van Dijk RB, Halfens RJ, Schols JM. Impact of Different Diagnostic Criteria on the Prevalence of Sarcopenia in an Acute Care Geriatric Ward. J Frailty Aging. 2014;3(4):222-9.

9. Gariballa S, Alessa A. Sarcopenia: prevalence and prognostic significance in hospitalized patients. Clin Nutr. 2013;32(5):772-6.

10. Sipers W, de Blois W, Schols J, van Loon LJC, Verdijk LB. Sarcopenia Is Related to Mortality in the Acutely Hospitalized Geriatric Patient. J Nutr Health Aging. 2019;23(2):128-37.

11. Vetrano DL, Landi F, Volpato S, Corsonello A, Meloni E, Bernabei R, et al. Association of sarcopenia with short- and long-term mortality in older adults admitted to acute care wards: results from the CRIME study. J Gerontol A Biol Sci Med Sci. 2014;69(9):115461.

12. Cederholm T, Jensen GL, Correia M, Gonzalez MC, Fukushima R, Higashiguchi T, et al. GLIM criteria for the diagnosis of malnutrition - A consensus report from the global clinical nutrition community. Clin Nutr. 2019;38(1):1-9.

13. Dent E, Morley JE, Cruz-Jentoft AJ, Arai H, Kritchevsky SB, Guralnik J, et al. International Clinical Practice Guidelines for Sarcopenia (ICFSR): Screening, Diagnosis and Management. J Nutr Health Aging. 2018;22(10):1148-61.

14. Sergi G, De Rui M, Stubbs B, Veronese N, Manzato E. Measurement of lean body mass using bioelectrical impedance analysis: a consideration of the pros and cons. Aging Clin Exp Res. 2017;29(4):591-7.

15. Calvani R, Picca A, Cesari M, Tosato M, Marini F, Manes-Gravina E, et al. Biomarkers for Sarcopenia: Reductionism vs. Complexity. Curr Protein Pept Sci. 2018;19(7):63942.

16. Ibrahim K, Howson FFA, Culliford DJ, Sayer AA, Roberts HC. The feasibility of assessing frailty and sarcopenia in hospitalised older people: a comparison of commonly used tools. BMC Geriatr. 2019;19(1):42.

17. Janssen I, Heymsfield SB, Baumgartner RN, Ross R. Estimation of skeletal muscle mass by bioelectrical impedance analysis. J Appl Physiol (1985). 2000;89(2):465-71.

18. Mijnarends DM, Schols JM, Meijers JM, Tan FE, Verlaan S, Luiking YC, et al. Instruments to assess sarcopenia and physical frailty in older people living in a community (care) setting: similarities and discrepancies. J Am Med Dir Assoc. 2015;16(4):301-8. 
19. Ward LC, Muller MJ. Bioelectrical impedance analysis. Eur J Clin Nutr. 2013;67 Suppl $1: \mathrm{S} 1$.

20. Kyle UG, Bosaeus I, De Lorenzo AD, Deurenberg P, Elia M, Gomez JM, et al. Bioelectrical impedance analysis--part I: review of principles and methods. Clin Nutr. 2004;23(5):1226-43.

21. Kyle UG, Bosaeus I, De Lorenzo AD, Deurenberg P, Elia M, Manuel Gomez J, et al. Bioelectrical impedance analysis-part II: utilization in clinical practice. Clin Nutr. 2004;23(6):1430-53.

22. Tanaka NI, Miyatani M, Masuo Y, Fukunaga T, Kanehisa H. Applicability of a segmental bioelectrical impedance analysis for predicting the whole body skeletal muscle volume. J Appl Physiol (1985). 2007;103(5):1688-95.

23. Kyle UG, Genton L, Karsegard L, Slosman DO, Pichard C. Single prediction equation for bioelectrical impedance analysis in adults aged 20--94 years. Nutrition. 2001;17(3):248-53.

24. Kyle UG, Genton L, Hans D, Pichard C. Validation of a bioelectrical impedance analysis equation to predict appendicular skeletal muscle mass (ASMM). Clin Nutr. 2003;22(6):537-43.

25. Drey M, Pfeifer K, Sieber CC, Bauer JM. The Fried frailty criteria as inclusion criteria for a randomized controlled trial: personal experience and literature review. Gerontology. 2011;57(1):11-8.

26. Fried LP, Tangen CM, Walston J, Newman AB, Hirsch C, Gottdiener J, et al. Frailty in older adults: evidence for a phenotype. J Gerontol A Biol Sci Med Sci. 2001;56(3):M146-56.

27. Bray C, Bell LN, Liang H, Haykal R, Kaiksow F, Mazza JJ, et al. Erythrocyte Sedimentation Rate and C-reactive Protein Measurements and Their Relevance in Clinical Medicine. WMJ. 2016;115(6):317-21.

28. Malnutrition Advisory Group BAPEN. Malnutrition Universal Screening Tool. 2008. p. 11,2 .

29. Katz S, Ford AB, Moskowitz RW, Jackson BA, Jaffe MW. Studies of Illness in the Aged. The Index of Adl: A Standardized Measure of Biological and Psychosocial Function. JAMA. 1963;185:914-9.

30. Nagaratnam N, Gayagay G, Jr. Validation of the Cumulative Illness Rating Scale (CIRS) in hospitalized nonagenarians. Archives of gerontology and geriatrics. 2007;44(1):29-36.

31. Kruizenga HM, Seidell JC, de Vet HC, Wierdsma NJ, van Bokhorst-de van der Schueren MA. Development and validation of a hospital screening tool for malnutrition: the short nutritional assessment questionnaire (SNAQ). Clinical nutrition (Edinburgh, Scotland). 2005;24(1):75-82.

32. Ling $\mathrm{CH}$, de Craen AJ, Slagboom PE, Gunn DA, Stokkel MP, Westendorp RG, et al. Accuracy of direct segmental multi-frequency bioimpedance analysis in the assessment of total body and segmental body composition in middle-aged adult population. Clin Nutr. 2011;30(5):610-5.

33. Chien MY, Huang TY, Wu YT. Prevalence of sarcopenia estimated using a bioelectrical impedance analysis prediction equation in community-dwelling elderly people in Taiwan. J Am Geriatr Soc. 2008;56(9):1710-5.

34. Hooper L, Abdelhamid A, Attreed NJ, Campbell WW, Channell AM, Chassagne P, et al. Clinical symptoms, signs and tests for identification of impending and current waterloss dehydration in older people. Cochrane Database Syst Rev. 2015(4):CD009647.

35. Thomas DR, Tariq SH, Makhdomm S, Haddad R, Moinuddin A. Physician misdiagnosis of dehydration in older adults. J Am Med Dir Assoc. 2004;5(2 Suppl):S30-4.

36. Fortes MB, Owen JA, Raymond-Barker P, Bishop C, Elghenzai S, Oliver SJ, et al. Is this elderly patient dehydrated? Diagnostic accuracy of hydration assessment using physical signs, urine, and saliva markers. J Am Med Dir Assoc. 2015;16(3):221-8.

37. Perez-Zepeda MU, Sgaravatti A, Dent E. Sarcopenia and post-hospital outcomes in older adults: A longitudinal study. Arch Gerontol Geriatr. 2017;69:105-9. 
38. Berger VA, Rousset P, MacCormack C, Ritz P. Reproducibility of body composition and body water spaces measurements in healthy elderly individuals. J Nutr Health Aging. 2000;4(4):243-5. 


\section{CHAPTER 5}

\section{The Martin Vigorimeter represents a reliable}

and more practical tool than the Jamar dynamometer to assess handgrip strength in the geriatric patient

Keywords: aging, physical function, functional capacity, strength, sarcopenia

Walther M.W.H. Sipers, Lex B Verdijk, Simone J.E. Sipers, Jos M.G.A. Schols, Luc JC van Loon Journal of the American Medical Directors Association. 2016 May1;17(5):466.e1-7 


\begin{abstract}
Background: Geriatric patients with low skeletal muscle mass and strength generally have a relative poor clinical outcome following acute illness. Therefore, it is recommended to routinely assess skeletal muscle mass and strength in patients admitted to the acute care geriatric ward. Handgrip strength is generally measured as a proxy for muscle strength and/or functional performance.
\end{abstract}

Objective: To compare the applicability and test-retest reliability of measuring handgrip strength using the Jamar dynamometer and the Martin Vigorimeter in geriatric patients during hospitalization.

Design: A total of 96 geriatric patients (age $85 \pm 5$ y) admitted to the acute geriatric ward participated in this study. Handgrip strength was assessed three times on two different occasions within one week of hospital admission using both the Jamar dynamometer and the Martin Vigorimeter.

Results: Maximal handgrip strength as determined over the three successive attempts performed on two occasions averaged $17 \pm 7 \mathrm{~kg}$ and $35 \pm 13 \mathrm{kPa}$ when using the Jamar dynamometer and Martin Vigorimeter, respectively. Handgrip strength was significantly greater when using the dominant versus non-dominant hand using both the Jamar dynamometer $(17 \pm 7 \mathrm{~kg}$ vs $16 \pm 7 \mathrm{~kg} ; P=0.003)$ and Martin Vigorimeter $(34 \pm 12 \mathrm{kPa}$ vs $33 \pm 13 \mathrm{kPa} ; P=0.022)$. Test-retest reliability showed an ICC of 0.94 and 0.92 when applying the Jamar dynamometer or Martin Vigorimeter, respectively (both $\mathrm{P}<0.001$ ). Furthermore, handgrip strength assessed with the Jamar and Martin Vigorimeter showed a strong correlation for both the first $(\rho=0.83$, $P<0.001)$ and second measurement $(\rho=0.79, P<0.001)$. Almost $80 \%$ of the geriatric patients needed help from nursing staff with transfer from bed to an arm rested chair measuring handgrip strength with the Jamar dynamometer according to the Southampton protocol which is not necessary when using the Martin Vigorimeter.

Conclusion: The Martin Vigorimeter represents a reliable and more practical tool than the Jamar dynamometer to assess handgrip strength in the geriatric patient upon admission to the acute geriatric ward. 


\section{Introduction}

Aging is associated with progressive loss of skeletal muscle mass and strength, commonly termed sarcopenia $(1,2)$. This age-related decline in skeletal muscle mass and strength impairs functional performance, leading to a decreased level of independence and an increased risk of developing chronic metabolic diseases (3). In the elderly patient skeletal muscle mass and strength are strong prognostic factors for their functional decline, morbidity, and mortality (4-6). Low skeletal muscle mass and poor physical performance are highly prevalent in hospitalized geriatric patients (7). Geriatric patients with little skeletal muscle mass and strength generally have a poor clinical outcome following acute illness $(8,9)$. Consequently, it is strongly recommended to routinely assess skeletal muscle mass and strength in patients admitted to the acute care geriatric ward.

Though there are various methods to assess skeletal muscle mass, strength, and functional capacity (10), most of these measurements are not practical or feasible to perform in the geriatric ward. Because of the simplicity of the measurement it is generally recommended to assess handgrip strength as a proxy for muscle strength and/or functional performance. Handgrip strength is usually assessed using the Jamar dynamometer (Figure 1), which was introduced by Bechtol (11). Mathiowetz et al. (12) demonstrated that standardized positions and instructions of use are important to allow a reliable measurement of handgrip strength when using the Jamar dynamometer. However, measuring handgrip strength with the Jamar dynamometer according to standardized protocol (13) is often complicated or simply impossible in geriatric patients admitted to the geriatric ward. In many cases, the predetermined sitting position in an arm rested chair is impossible when patients are bedridden due to physical impairments. Furthermore, many geriatric patients frequently have painful arthritic hands, which makes handling of the $1.5 \mathrm{~kg}$ weighing Jamar dynamometer challenging.

Another instrument to measure handgrip strength is the Martin Vigorimeter (Figure 1) (14). The Martin Vigorimeter is a pseudo-dynamic dynamometer that measures the pressure when subjects press a rubber bulb connected by a tube to a manometer, with pressure being expressed in kiloPascal ( $\mathrm{kPa})(15)$. The Martin Vigorimeter has been specifically designed for arthritic subjects to allow proper grip strength assessment while avoiding excess stress on weak or painful joints $(12,16)$. Though the use of this Martin Vigorimeter is not as widespread as the Jamar, it has been frequently used to assess handgrip strength in clinical practice (13, $15,17-23)$. An evaluation of handgrip strength in a healthy older population via the use of the Jamar handheld dynamometer versus the Martin Vigorimeter has confirmed the overall applicability of both methods in the elderly population $(13,18)$. 


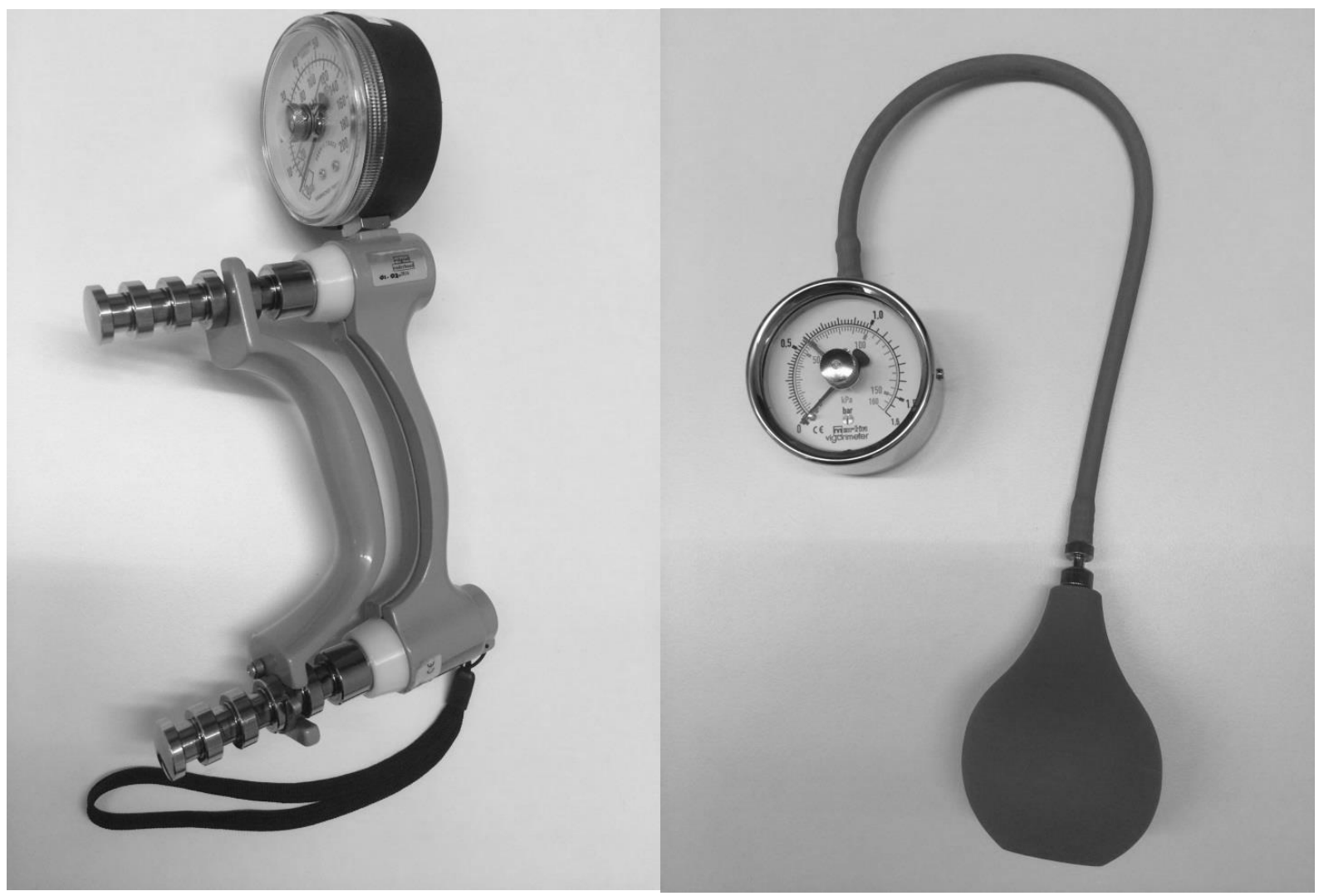

Figure 1: Jamar dynamometer (left) and Martin Vigorimeter (right) (photo W. Sipers (C)

In clinically compromised geriatric patients, the use of the Martin Vigorimeter allows the assessment of handgrip strength with minimal requirements for position and optimal capacity for the patients to generate maximal grip in a (for them) comfortable position. We hypothesize that the Martin Vigorimeter is a reliable and more practical tool to assess handgrip strength in the geriatric patient, with test-retest reliability comparable or even better than when using the Jamar dynamometer.

The present study evaluates the applicability and test-retest reliability of measuring handgrip strength with both the Jamar handheld dynamometer and the Martin Vigorimeter in geriatric patients during hospitalization. A total of 109 geriatric patients were selected upon admission to an acute care geriatric ward of a teaching hospital. Handgrip strength was measured three times with both devices on two separate occasions in acutely ill geriatric patients within one week of hospital admission.

\section{Methods}

\section{Study sample}

All geriatric patients admitted to the acute geriatric ward of a large Dutch general hospital were asked to participate in the study. For organizational reasons, there were three periods of 
recruitment: the first from March through June 2012 and the second and third for the same months in 2013 and 2014. The inclusion criteria were: age above $70 \mathrm{y}$ and being frail according to the Groningen Frailty Indicator (GFI) (24). Patients were excluded if they had hand deformation, missing fingers, acute arthritis or a fracture of the wrist or hand, and/or were not able to follow instructions because of a severe confusional state or dementia. All patients were informed on the nature of the measurements before written informed consent was obtained from the patient or proxy. This study complied with the guidelines set out in the Declaration of Helsinki and was approved by the Ethics Committee of Sittard-Heerlen, the Netherlands (number 13-N-60).

\section{Patients' characteristics}

Patient characteristics were retrieved from the medical and nursing files. These included sex, age, living situation, diagnosed medical conditions, medical history and activities of daily living prior to the acute illness that led to hospital admission. Height was estimated to the nearest $\mathrm{cm}$ by measuring ulna length because many patients were temporarily bedridden (25). The frailty score was assessed according to the GFI criteria, which ranges from 0 to 15: a score of 4 or higher indicates frailty $(24,26,27)$. Bodyweight was measured on a sitting weight scale (SECA, Model 959).

A number of scales were used to evaluate patient status. The cumulative illness rating scale (CIRS) was used to calculate the number and severity of chronic illnesses of the patients' comorbid diseases. The score ranges from 0 , which corresponds to the absence of disorders, to a maximum of 56 (28). Malnutrition was measured using the Short Nutritional Assessment Questionnaire (SNAQ), which is a validated screening instrument for malnutrition. Scores range from 0 to 5 ; a score of 3 or higher indicates that the patient is malnourished (29). The Katz ADL-6, a validated instrument for screening daily living activities, was used to assess ADL. Scores range from 0 ,totally independent, to 6 , representing completely dependent $(30$, 31).

The Short Physical Performance Battery (SPPB) and the Hierarchical Balance and Mobility (HABAM) were measured for mobility. The SPPB consists of 3 parts, balance, gait speed and chair stand test. The range is from 0 to 12 points (32) with the highest score representing the best performance. Volpato et al. showed that the SPPB has predictive value in hospitalized elderly (33). The HABAM was developed in the 1990's by MacKnight and Rockwood and gives information about balance, transfers and mobility. The higher the score the better the mobility level, with scores ranging from 0-67 (34). Hubbard et al. showed that the HABAM provides useful information about disease progression in hospitalized elderly (35). The Maltron BioScan 920-II, a multi-frequency multi-segmental bio-impedance (mf-ms BIA) device, was used to measure muscle mass. Mf-ms BIA has been validated for the assessment of whole body 
composition and segmental lean mass in elderly people. The Maltron BioScan 920-II has an eight-point electrode system, which separately measures impedance of the patient's trunk, arms and legs at four different frequencies $(5 \mathrm{kHz}, 50 \mathrm{kHz} 100 \mathrm{~Hz}$ and $200 \mathrm{~Hz}$ ) for each body segment. The Maltron BioScan 920-II calculates appendicular skeletal muscle mass (SMM) according to the device-specific calculation called the Maltron calculation (36). Patients were measured early in the morning before breakfast, wearing only the pajamas, as described in the user's manual. Skeletal muscle mass index (SMI: MM/height ${ }^{2}$ ) and relative muscle mass (RMM: MM/weight) were calculated skeletal muscle mass parameters (1).

The Mini Mental State Examination (MMSE) was used to assess cognitive status. It is an 11question measure that tests five areas of cognitive function: orientation, registration, attention and calculation, recall, and language. The maximum score is 30 . A score of 23 or lower is indicative of cognitive impairment. The MMSE takes only 5-10 min to administer and is therefore practical to use repeatedly and routinely (37).

\section{Handgrip strength measurements}

The Jamar dynamometer (Sammons Preston, Inc., Bolingbrook, IL, USA) was introduced by Bechtol (11) and is a frequently used and validated device for assessing handgrip strength in healthy elderly people. Calibration of the Jamar dynamometer was performed before, during and after cessation of the study according to the guidelines set by the manufacturer. The Martin Vigorimeter (14) was developed in Europe and is composed of a rubber bulb which is grasped by the hand. The pressure in the bulb is registered on a manometer via a rubber junction tube. The Martin Vigorimeter was newly purchased prior to performing the tests.

Handgrip strength was assessed with the second handle position of the Jamar dynamometer and the largest bulb was used for the Martin Vigorimeter. We applied the Southampton protocol (13) if the patient was able to sit on an armchair ( $n=68$ and 71 for first and second set of measurements, respectively). Otherwise the patient was sitting on the bed edge ( $n=5$ and 9 for first and second set of measurements, respectively), or lying in bed ( $n=23$ and 16 for first and second set of measurements, respectively). If the patient was bedridden, a sitting position was chosen with the forearm rested in neutral position on the abdomen and with the wrist in 0 to 30 degrees of extension. The position during measurement was identical between the Jamar dynamometer and Martin Vigorimeter.

Three grip strength measurements were performed for each hand with both instruments, with a rest period of $30 \mathrm{~s}$ between successive attempts. Both hands were tested, the dominant first. All participants were randomly assigned a number. Subjects with an even number started with the Jamar and subjects with uneven number started with the Martin Vigorimeter. Every patient performed two sets of measurements within one week after hospital admission, $\sim 6$ days apart. All measurements were performed between 11 am and $2 \mathrm{pm}$. Hand dominance was 
estimated with Edinburgh handedness inventory (38), and hand size (length and circumference) were determined. Hand circumference was measured at the thumb commissure following the axis of the head of the metacarpals. The hand length was measured from distal crease of the wrist to the distal extremity of the middle finger.

\section{Statistics}

Patients' characteristics are described by mean $\pm S D$ and range for continuous variables and by frequencies and percentages for the categorical variables. The variability between the three attempts within each set of measurement was determined by calculating the coefficient of variation. Repeated measures ANOVA was used to determine systematic differences between the three attempts. Paired t-tests were used to compare each attempt separately (i.e. first, second, and third attempt) with the maximum score over the three attempts. Similarly, paired ttests were used to determine potential differences between dominant and non-dominant hand. All these analyses were performed for the Jamar handheld dynamometer and Martin Vigorimeter separately, and for the first and second measurement (one week apart) separately. To determine test-retest reliability between the first and second set of measurements (one week apart), intraclass correlation coefficients (ICC for absolute agreement and average measures) were calculated for both the Martin Vigorimeter and Jamar separately. To provide further insight in the test-retest reliability for smaller subgroups of patients, ICC was also calculated separately for: bed-ridden and non-bed-ridden patients; patients in the lowest and highest quartile of handgrip strength (determined by Jamar); and patients with a GFI above or below 7 .

To test the agreement between both methods of strength assessment, handgrip strength as measured with the Jamar was correlated with handgrip strength measured with the Martin Vigorimeter, at both time points separately. Correlation analyses were also performed to assess the relation between handgrip strength and: age, weight, height, hand length, hand circumference, hand problems (e.g., missing finger and osteoarthritic pain), presence of an IV needle, MMSE, SNAQ score, Katz score, GFI score, presence of delirium, SPPB score, HABAM score, CRP, creatinine, skeletal muscle mass parameters (MM, SMI and RMM), both for the Jamar handheld dynamometer and the Martin Vigorimeter. Spearman's rho was calculated since handgrip strength was not normally distributed based on Kolmogorov-Smirnov testing. To determine the relation between handgrip strength and gender, unpaired t-tests were performed comparing men and women. Finally, linear regression analysis was performed to determine which of the above factors could best predict handgrip strength. Based on the results of the correlation analysis, a number of independent variables were included as potential predictors: gender, height, weight, hand circumference, skeletal muscle mass, KATZ, SPPB and HABAM. 


\section{Results}

\section{Patients}

In the 3 three-month periods, 282 older patients were admitted to the acute geriatric ward and asked to participate. Thirteen patients or proxies refused to participate. Of the remaining 269 patients, 160 were excluded from the present analysis, because only one set of measurements was performed due to early hospital discharge. Another 13 patients were excluded because of hand problems like missing fingers or hand deformation, leaving a total of 96 patients (64\% female) with complete datasets that were included in the analyses (Table 1).

Table 1: Patients' characteristics, $n=96(\%)$

\begin{tabular}{|c|c|c|}
\hline Women, $n$ & & $61(64)$ \\
\hline Men, $n$ & & $35(36)$ \\
\hline Age, $y$ & & $85 \pm 5$ \\
\hline Height, $\mathrm{cm}$ & & $162 \pm 8$ \\
\hline Weight, $k g$ & & $67 \pm 13$ \\
\hline Living in community, $n$ & & $78(81)$ \\
\hline Assisted living, $n$ & & $18(19)$ \\
\hline GFI score & & $7.1 \pm 3.0$ \\
\hline MMSE & & $18.6 \pm 5.2$ \\
\hline CIRS score & & $20.0 \pm 5.5$ \\
\hline \multirow[t]{7}{*}{ Katz ADL 6-score $(n=94)$} & 0 & $13(14)$ \\
\hline & 1 & $17(18)$ \\
\hline & 2 & $5(5)$ \\
\hline & 3 & $13(14)$ \\
\hline & 4 & $7(7)$ \\
\hline & 5 & $16(17)$ \\
\hline & 6 & $23(25)$ \\
\hline \multirow[t]{3}{*}{ SNAQ score $(n=93)$} & $0-1$ & $48(52)$ \\
\hline & 2 & $6(6)$ \\
\hline & $\geq 3$ & $39(42)$ \\
\hline
\end{tabular}

GFI: Groningen Frailty Indicator. MMSE: Mini Mental State Examination. CIRS: Cumulative IIIness Rating Scale. SNAQ: Short Nutritional Assessment Questionnaire Data represent total $n(\%)$, or means $\pm S D$.

A flow diagram of patient recruitment is provided in Supplementary figure 1. Mean age of participants was $85 \pm 5$ y and $81 \%$ of the patients lived in the community, in the surroundings of the hospital. All patients were frail with a mean GFI score of $7.1 \pm 3$.0. The mean MMSE score was $18.6 \pm 5.2$ indicating moderate cognitive problems. The CIRS score was $20.0 \pm 5.5$. Fortytwo percent of the participants were malnourished, with SNAQ scores of 3 or higher. Forty-two percent were highly ADL dependent, with a Katz ADL- 6 score of 5 or 6 . Eighty-seven patients were right dominant and 9 were left dominant. The mean time between the first and second set of measurements was $6.3 \pm 3.3$ days. 
Handgrip strength with Jamar dynamometer

No systematic differences were observed between the first, second and third attempt for the Jamar handheld dynamometer at both measurements. Moreover maximal handgrip strength as determined over the three successive attempts was significantly greater compared to each of the 3 attempts separately, for both the dominant and non-dominant hand $(P<0.001)$. Maximal handgrip strength assessed with the dominant hand was significantly greater when compared to the non-dominant hand at baseline $(17 \pm 7$ vs $16 \pm 7 \mathrm{~kg} ; P=0.003)$ and one week later $(17 \pm 7$ vs $16 \pm 7 \mathrm{~kg} ; P<0.001)$. In addition, maximal handgrip strength was significantly lower in the women vs men in both the dominant and non-dominant hand at baseline $(15 \pm 6$ vs $21 \pm 7 \mathrm{~kg}$ and $14 \pm 6$ vs $21 \pm 7 \mathrm{~kg}$, respectively; $P<0.001$ ), and one week later (data not shown). The COV calculated over the three attempts for the dominant and non-dominant hand, and for the first and second measurement separately, ranged from $9-11 \%$ for the Jamar dynamometer (Table 2).

Maximal handgrip strength assessed with the dominant hand and non-dominant hand did not change significantly approximately one week later compared with the first measurement with Jamar dynamometer $(0.05 \mathrm{~kg}$ for both dominant and non-dominant hand; Table 2; $P=0.888$ and $P=0.867$, respectively).

Table 2: Maximal handgrip strength in hospitalized geriatric patients $(n=96)$

\begin{tabular}{lcccc}
\hline \multicolumn{1}{c}{ T0 Max } & T0 COV (\%) & T1 Max & T1 COV (\%) \\
\hline Vigori dom, $k P a$ & $34 \pm 12(2-70)$ & $9.6 \pm 8.8$ & $35 \pm 13(2-73)$ & $7.8 \pm 6.0$ \\
Vigori non-dom, $k P a$ & $33 \pm 13(2-70)^{*}$ & $8.2 \pm 7.9$ & $33 \pm 13(3-69)^{*}$ & $8.6 \pm 8.8$ \\
Jamar dom, $k g$ & $17 \pm 7(2-33)$ & $10.2 \pm 9.4$ & $17 \pm 7(1-35)$ & $9.2 \pm 10.7$ \\
Jamar non-dom, $k g$ & $16 \pm 7(4-33)^{*}$ & $9.5 \pm 6.5$ & $16 \pm 7(2-33)^{\star}$ & $9.1 \pm 7.6$ \\
\hline
\end{tabular}

Dom: dominant hand. Non-dom: non-dominant hand. T0: assessment at hospital admission. T1: assessment after an average of 6.3 days in hospital. Max: maximal score determined over 3 successive attempts. COV: coefficient of variation (\%) determined over 3 successive attempts. Data represent means $\pm S D$ (range) for maximal handgrip strength and COV. : significantly different from dominant hand $(P<0.001)$.

Correlation and regression analyses were performed using maximal handgrip strength of the dominant hand. Handgrip strength measured with the Jamar dynamometer correlated significantly with height, weight, hand circumference, hand length and Katz score (Supplementary table 1). There was a trend for a correlation between handgrip strength and age and GFI, but no significant correlations were observed between handgrip strength and CIRS.

Based on the correlation analyses, gender, age, weight, height, GFI, hand circumference and KATZ were included as potential predictors for handgrip strength in a linear regression model. Forward regression analysis was performed on data for $n=84$ geriatric patients. The height and 
KATZ significantly predicted handgrip strength measured with the Jamar dynamometer, accounting for $31 \%$ of the variability (Table 3 ).

Table 3: Potential predictors for handgrip strength in hospitalized geriatric patients $(n=84)$

\begin{tabular}{|c|c|c|c|c|c|c|}
\hline (2) & \multicolumn{3}{|c|}{ Martin Vigorimeter } & \multicolumn{3}{|c|}{ Jamar dynamometer } \\
\hline $\begin{array}{l}\mathbf{R}^{2} \\
P_{\text {-valu }}\end{array}$ & & $\begin{array}{r}0.302 \\
<0001\end{array}$ & & & $\begin{array}{c}0.314 \\
<0.001\end{array}$ & \\
\hline $\begin{array}{l}\text { Beta Standardized } \\
\text { coefficient }\end{array}$ & $\begin{array}{l}\text { Height } \\
\text { KATZ } \\
\text { Hand circ. }\end{array}$ & $\begin{array}{c}0.315 \\
-0.304 \\
0.206\end{array}$ & $\begin{array}{l}P=0.002^{* *} \\
P=0.002^{* *} \\
P=0.039^{*}\end{array}$ & $\begin{array}{l}\text { Height } \\
\text { KATZ }\end{array}$ & $\begin{array}{c}0.452 \\
-0.306\end{array}$ & $\begin{array}{l}P<0.001^{* *} \\
P=0.001^{* *}\end{array}$ \\
\hline
\end{tabular}

Forward Linear Regression analysis for maximum handgrip strength of the dominant hand measured at T0 for both the Jamar dynamometer and Martin Vigorimeter. Gender, age, weight, height, GFI hand circumference and KATZ were included as potential predictors for handgrip strength. *: $P<0.05$; **: $P<0.001$.

In a subgroup, additional data were available of MMSE $(n=67)$, SPPB $(n=51), \operatorname{HABAM}(n=45)$, hand pain $(n=40)$, and skeletal muscle mass parameters (SMM, SMI and RMM) $(n=37)$. We observed that maximal handgrip strength in the dominant hand was strongly correlated with SPPB, HABAM $(P<0.05)$ and SMM $(P<0.001)$, but not with MMSE, hand pain and skeletal muscle mass parameters like SMI and RMM (Supplementary table 1).

Forward linear regression was performed including SPPB, HABAM and SMM as potential predictors on a subgroup of $n=32$ geriatric patients. In this analysis, SMM and SPPB significantly predicted handgrip strength measured with the Jamar dynamometer, accounting for $44 \%$ of the variability (Supplementary table 2 ).

\section{Handgrip strength with Martin Vigorimeter}

No systematic differences were observed between the first, second and third attempt for the Martin Vigorimeter at both measurements. Maximal handgrip strength as determined over the three successive attempts was significantly greater compared to each of the 3 attempts separately, for both the dominant and non-dominant hand for the Martin Vigorimeter $(P<0.001)$. Moreover maximal handgrip strength assessed with the dominant hand was significantly greater when compared to the non-dominant hand at baseline $(34 \pm 12$ vs $33 \pm 13$ $\mathrm{kPa} ; P=0.022)$ and one week later $(35 \pm 13$ vs $33 \pm 13 \mathrm{kPa} ; P=0.017)$. In addition, maximal handgrip strength was significantly lower in the women vs men in both the dominant and nondominant hand at baseline ( $31 \pm 11$ vs $41 \pm 13 \mathrm{kPa}$ and $29 \pm 11$ vs $40 \pm 14 \mathrm{kPa} ; P<0.001)$ and one week later (data not shown).

The COV calculated over the three attempts for the dominant and non-dominant hand, and for the first and second measurement separately, ranged from 8-10 \% for the Martin Vigorimeter (Table 2). Maximal handgrip strength assessed with the dominant hand and non-dominant hand did not differ when assessed one week later compared with the first measurement with Martin Vigorimeter ( 0.4 and $0.3 \mathrm{kPa} ; P=0.522$ and $P=0.616$, respectively). 
Handgrip strength of the dominant hand measured with Martin Vigorimeter correlated significantly with height, weight, hand circumference, hand length and Katz score (Table 3). There was a trend for a correlation between handgrip strength measured with Martin Vigorimeter and age and GFI but there was no correlation with CIRS. Based on the correlation analyses, gender, age, weight, height, GFI, hand circumference and KATZ were included as potential predictors for handgrip strength in a linear regression model. Forward regression analysis was performed on data for $n=84$ geriatric patients. Height, KATZ and hand circumference predicted $30 \%(P<0.001)$ of the handgrip strength measured with the Martin Vigorimeter (Table 3).

In the subgroup of patients for whom additional data were available, maximal handgrip strength in the dominant hand measured with the Vigorimeter was strongly correlated with SPPB $(P<0.05)$, HABAM and SMM $(P<0.001)$, but not with MMSE, hand pain and skeletal muscle mass parameters like SMI and RMM (Supplementary table 1). Forward linear regression was performed including SPPB, HABAM and SMM as potential predictors on a subgroup of $n=32$ geriatric patients. In this analysis, SMM and HABAM predicted $43 \%$ $(P<0,001)$ of the handgrip strength measured with the Martin Vigorimeter (Supplementary table 2).

\section{Jamar dynamometer vs Martin Vigorimeter}

Test-retest reliability for maximal handgrip strength of the dominant hand was shown to be high for both the Jamar dynamometer $(\mathrm{ICC}=0.94, P<0.001)$ and Martin Vigorimeter $(\mathrm{ICC}=0.92$, $P<0.001)$. The test-retest reliability of the handgrip strength of the dominant hand remained high in both the geriatric patients who were $(n=68)$ and were not able to sit on an arm rested chair during the measurement $(n=28)$, with an ICC of 0.88-0.95 (Supplementary table 3; all $P<0.001)$. Similarly, test-retest reliability of the handgrip strength of the dominant hand measured with both Jamar dynamometer and Martin Vigorimeter remained high in patients within the lowest and highest quartiles of handgrip strength, and in patients with a frailty (GFI) index above or below 7 (Supplementary table 3).

Apart from high test-retest reliability, a strong correlation was observed between the Jamar dynamometer and Martin Vigorimeter for both the first $(\rho=0.83, P<0.001)$ and second measurement ( $\rho=0.79, P<0.001)$ (Figure 2).

To further support the use of handgrip strength as an indicator of physical functioning, handgrip strength was compared between patients of different functional level based on the Katz ADL-6 score. 


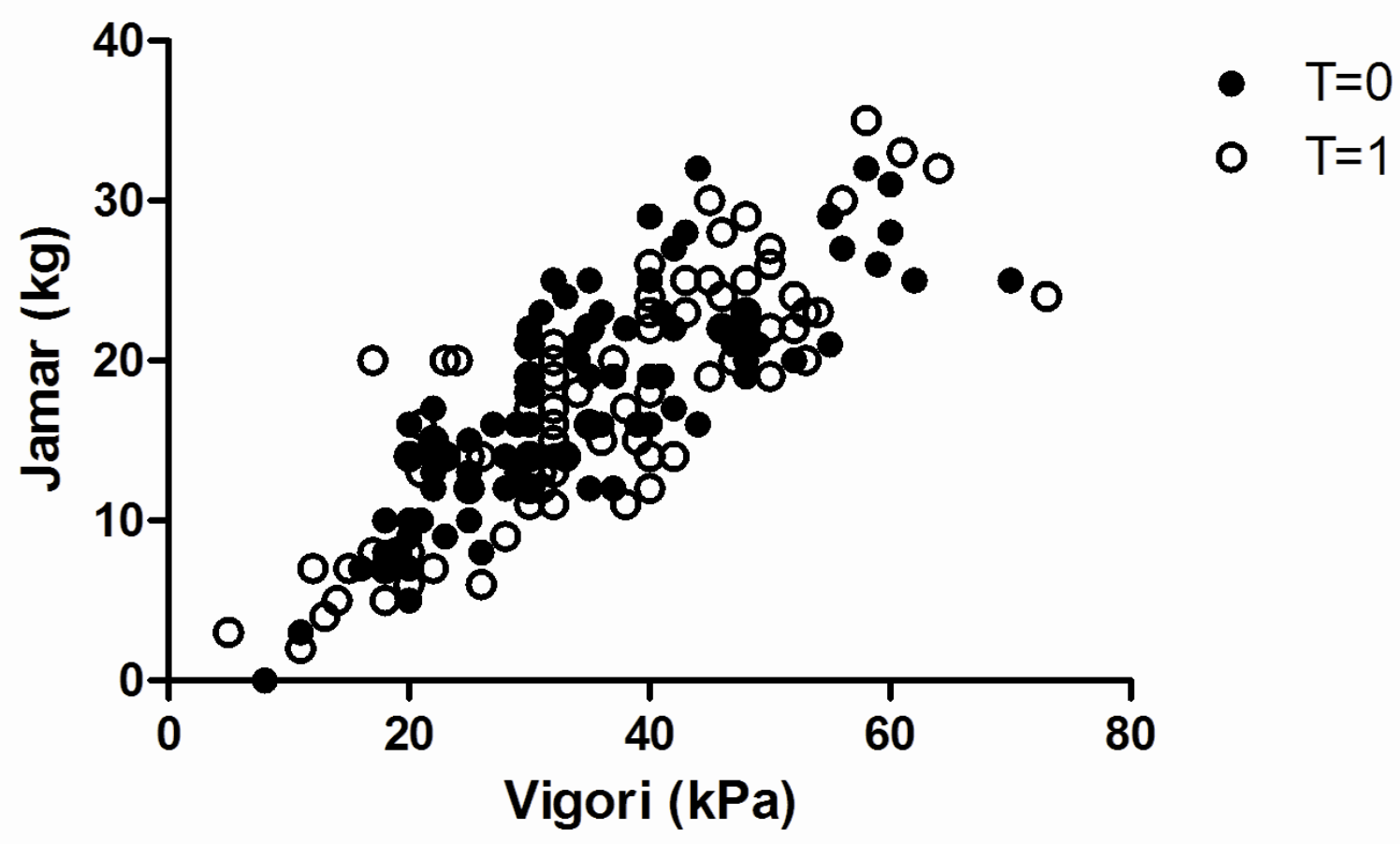

Figure 2: Distribution of the correlation between maximal handgrip strength in geriatric patients $(n=96)$ measured with the Jamar dynamometer and the Martin Vigorimeter for the dominant hand at baseline and one week later.

In agreement with the observed correlation between the Jamar dynamometer and Martin Vigorimeter, handgrip strength was significantly lower in those patients with a low level of functional independence compared with patients with a higher level of functional independence when assessed with both the Jamar dynamometer and Martin Vigorimeter (Figure 3).

When applying the Southampton protocol when measuring handgrip strength with the Jamar dynamometer the patient needs to transfer from bed to chair. In a subgroup $n=45$, data about mobility were available. $78 \%$ of these geriatric patients needed help from the nursing staff to allow a transfer from bed to a chair (HABAM-transfer score $<18$ ).

Furthermore, 8 patients complained about pain during the handgrip strength assessment using the Jamar handheld dynamometer whereas no patients complained when using the Martin Vigorimeter in a subgroup of $n=40$ in which presence of pain was assessed. Both from the patients' and nursing staff perspectives, the use of the Jamar Vigorimeter was more practical when compared to the Jamar dynamometer. 

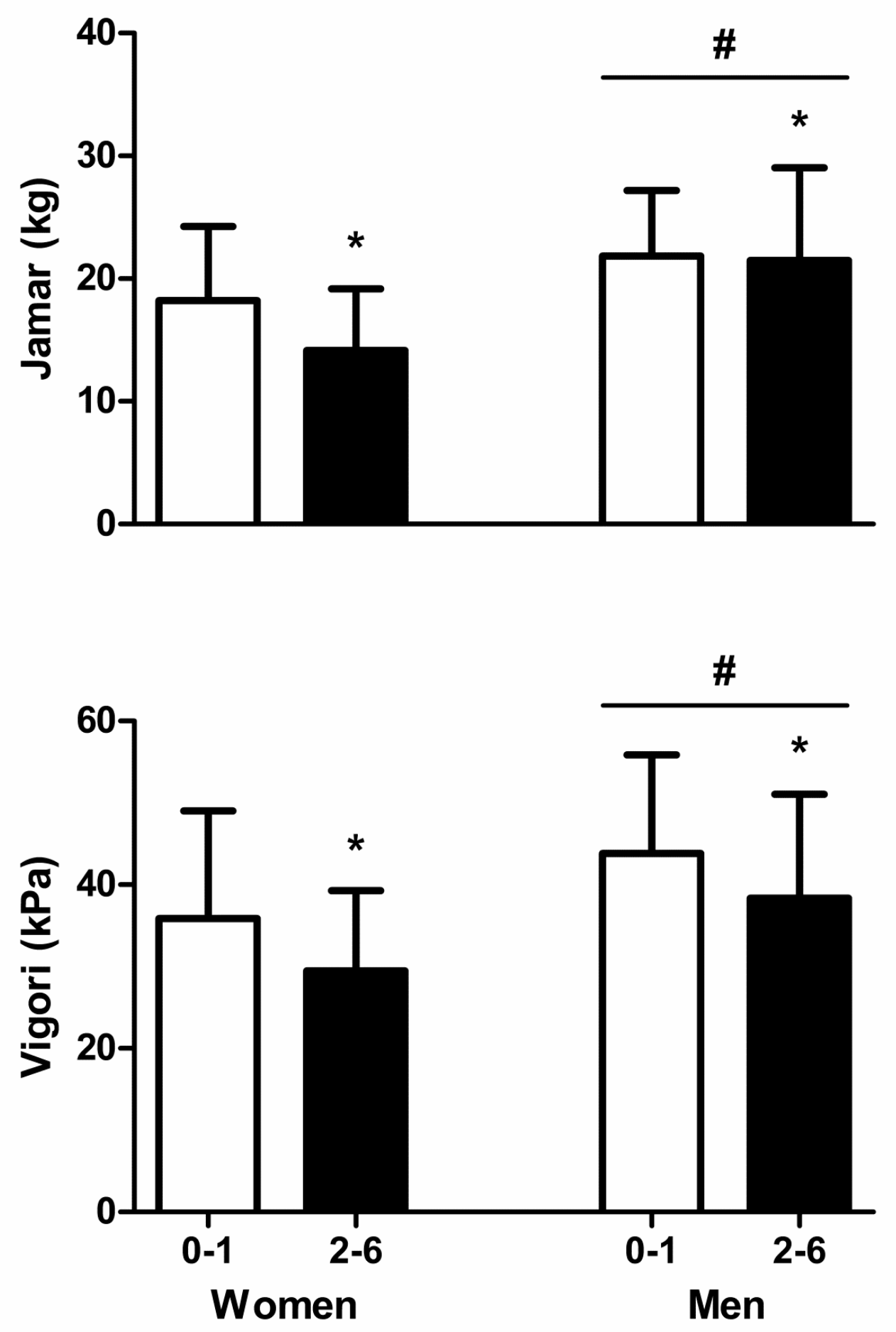

Figure 3: Maximal handgrip strength in the dominant hand with Jamar dynamometer (top) and Martin Vigorimeter (bottom) for geriatric patients with a high (Katz ADL-6 score 0-1; white bars; women: $n=18$ and men: $n=12$ ) and low (Katz ADL-6 score 2-6; black bars; women: $n=45$ and men: $n=19)$ level of functional independence. Data were analyzed using 2-way ANOVA with Katz ADL-6 score and gender as fixed factors. No Katz ADL-6 x gender interactions were observed. *: significantly different from Katz ADL-6 0-1 (main effect of Katz ADL-6 score for both Jamar and Vigorimeter $P<0.05$ ). \#: significantly different from women (main effect of gender for both Jamar and Vigorimeter $P<0.05)$ 


\section{Discussion}

In this study handgrip strength was measured using both the Jamar handheld dynamometer and the Martin Vigorimeter in 96 geriatric patients with moderate cognitive problems and of whom more than $40 \%$ were malnourished and highly ADL dependent. Nearly $25 \%$ of the geriatric patients were not able to sit on an arm rested chair during handgrip measurement due to acute illness. We demonstrated that the test-retest reliability for measuring handgrip strength with the Martin Vigorimeter is high (ICC0.92; $P<0.001)$ and comparable to the Jamar handheld dynamometer (ICC $0.94 ; P<0.001)$ in a clinically compromised population of acutely ill geriatric patients.

Geriatric patients with low skeletal muscle mass and strength generally have a poor clinical outcome following acute illness $(8,9)$. Handgrip strength assessment has been identified as a proxy for functional decline, morbidity and mortality (4-6) and is therefore often measured in geriatric patients using the Jamar dynamometer (13). The fact that acutely ill hospitalized geriatric patients often have osteoarthritis, reduced mobility, are frequently bedridden and care dependent (39) makes a reliable measurement of handgrip strength impossible or at least challenging. In this study we assessed handgrip strength using the Jamar dynamometer in 96 geriatric patients with a mean age of $85 \pm 5$ y (64 female, 32 males) with moderate cognitive problems and of whom more than $40 \%$ were malnourished and highly ADL dependent. As predicted, the assessment of handgrip strength in these patients using the Jamar dynamometer was complicated. Application of the Southampton protocol was not possible in 28 and 25 patients, respectively, upon hospital admission and one week later. Complaints of pain were reported in 8 out of $40(20 \%)$ patients during squeezing the Jamar handheld dynamometer. Maximal handgrip strength determined with the Jamar handheld dynamometer averaged $16 \pm 7 \mathrm{~kg}$ in the dominant hand and did not differ between the three successive measurements. These findings are comparable to previous observations by Desrosiers et al. (18) but absolute strength was much lower in our patients. The latter is not surprising as our population can be classified as more frail and/or sarcopenic. In our findings, test-retest reliability for maximal handgrip strength of the dominant hand (ICC: 0.94) was shown to be at least comparable with earlier findings in healthy adult (ICC: 0.80) (12) and healthy older (ICC: 0.91) American community-dwelling volunteers (40). We extend on this previous work by showing proper test-retest reliability when assessing handgrip strength using the Jamar dynamometer in geriatric patients admitted to the acute geriatric ward. However, in $20-40 \%$ of the patients this procedure was either impossible and/or impractical with substantial discomfort to the patient.

The Martin Vigorimeter represents another tool to assess handgrip strength. It is a pseudodynamic dynamometer consisting a rubber bulb connected by a tube to a manometer (15) and has been specifically designed for arthritic subjects to allow proper grip strength assessment 
while avoiding excessive stress on weak or painful joints $(12,16)$. Assessing handgrip strength in the geriatric patients using the Martin Vigorimeter was perceived as much more comfortable both by the patients and nursing staff. In line with the Jamar dynamometer, handgrip strength determined with the Martin Vigorimeter did not differ between the three successive measurements, but maximal handgrip strength $(34 \pm 12 \mathrm{kPa}$ in the dominant hand) was greater than each separate attempt. Handgrip strength assessment with the Martin Vigorimeter showed proper test-retest reliability (ICC: 0.92), that was comparable to previous reports on test-retest reliability of handgrip strength testing using the Martin Vigorimeter in elderly patients with rheumatic arthritis (41) and primary school children (42). Again, we extend on previous work by demonstrating good test-retest reliability for measuring handgrip strength in geriatric patients when using the Martin Vigorimeter, with values similar to the Jamar dynamometer (ICC 0.92 vs 0.94 ; both $\mathrm{P}<0.001$ ). In this study we show that applying the Jamar dynamometer as the golden standard is complicated in the compromised geriatric population. The Southampton protocol (13) is recommended when applying the Jamar dynamometer but is impractical and frequently too uncomfortable to the patients and nursing staff. Almost $25 \%$ of the 96 geriatric patients had moderate cognitive problems, were highly ADL dependent and most importantly, were not able to sit on an arm rested chair during handgrip strength measurement due to acute illness. Almost $80 \%$ of the geriatric patients needed help from the nursing staff to achieve such a transfer from bed to a chair. Furthermore complaints of pain were reported in 8 out of $40(20 \%)$ patients during squeezing the Jamar dynamometer due to hand osteoarthritis. Most (43\%) geriatric patients preferred the Martin Vigorimeter and the minority $(22 \%)$ the Jamar dynamometer. Handgrip strength measurement was therefore more comfortable when the Martin Vigorimeter was used. One of the main benefits was the lack of requirement to move from bedrest to a sitting position in an arm rested chair. Moreover complaints of pain were not reported when using the Martin Vigorimeter. As such, the Martin Vigorimeter represents a valid, reliable, and practical tool to assess handgrip strength in geriatric patients in both a clinical and research setting. Similar to the need of regular calibration of the Jamar dynamometer (43), it is obvious that regular inspection of the Martin Vigorimeter (to check for e.g. aging of the rubber bulb) is essential to maintain its excellent measurement properties. This will not only maintain proper test-retest reliability, but would also enable valid comparison of findings from different Vigorimeters within and between institutions. Given the somewhat different nature of force application between the Jamar and the Martin Vigorimeter, it remains to be determined whether the clinical/functional relevance of both measures is comparable. However, based on the strong correlation between both measurements, and their association with functional parameters (Supplementary table 1), we argue that the clinical relevance will likely not be much different between both measures. 


\section{Conclusion}

Taken together, for clinical practice, handgrip strength can be properly assessed upon hospital admission and subsequently followed up over time using both the Martin Vigorimeter and Jamar dynamometer. The measurement protocol should include maximal handgrip strength of 3 attempts of the dominant hand. Handgrip strength measurements with the Martin Vigorimeter showed similar high test-retest reliability (ICC and COV) when compared with the use of the Jamar dynamometer. Because of the practical issues and discomfort when using the Southampton protocol in the compromised geriatric patient admitted to the acute care geriatric ward, we strongly recommend the routine use of the Martin Vigorimeter procedure to assess handgrip strength. However, despite good measurement properties of the Martin Vigorimeter in this frail geriatric population there is still limited information about good cut-off points relevant for clinical decisions and treatment strategies (44). Therefore, we invite geriatricians and researchers to assess handgrip strength with the Martin Vigorimeter and, as such, to further establish clinically relevant cut-off points of handgrip strength with the Martin Vigorimeter in geriatric patients.

In conclusion, the Martin Vigorimeter forms a reliable and more practical tool than the Jamar dynamometer to measure handgrip strength in specific conditions in which patients are bedridden and/or otherwise compromised to perform handgrip strength measurement with the Jamar dynamometer according to standard protocol. Handgrip strength assessment with the Martin Vigorimeter can be performed in bed with less discomfort and pain when compared with the strength assessment using the Southampton protocol using the Jamar handheld dynamometer. 


\section{References}

1. Cruz-Jentoft AJ, Baeyens JP, Bauer JM, Boirie Y, Cederholm T, Landi F, et al. Sarcopenia: European consensus on definition and diagnosis: Report of the European Working Group on Sarcopenia in Older People. Age Ageing. 2010;39(4):412-23.

2. Rosenberg I. Summary comments: epidemiological and methodological problems in determining nutritional status of older persons. . American Journal Clinical Nutrition. 1989;50:1231-3.

3. Evans W. Functional and metabolic consequences of sarcopenia. The Journal of nutrition. 1997;127(5 Suppl):998S-1003S.

4. Giampaoli S, Ferrucci L, Cecchi F, Lo Noce C, Poce A, Dima F, et al. Hand-grip strength predicts incident disability in non-disabled older men. Age Ageing. 1999;28(3):283-8.

5. Metter EJ, Talbot LA, Schrager M, Conwit R. Skeletal muscle strength as a predictor of all-cause mortality in healthy men. J Gerontol A Biol Sci Med Sci. 2002;57(10):B35965.

6. Rantanen T, Avlund K, Suominen H, Schroll M, Frandin K, Pertti E. Muscle strength as a predictor of onset of ADL dependence in people aged 75 years. Aging Clin Exp Res. 2002;14(3 Suppl):10-5.

7. Sipers W.M.W.H MJMM, van Dijk R.B. , Halfens R.J.G., Schols J.M.G.A. Impact of different diagnostic criteria on the prevalence of sarcopenia in an acute care geriatric ward Journal of frailty and aging. 2014;03(04):222-9.

8. Gariballa S, Alessa A. Sarcopenia: prevalence and prognostic significance in hospitalized patients. Clin Nutr. 2013;32(5):772-6.

9. Vetrano DL, Landi F, Volpato S, Corsonello A, Meloni E, Bernabei R, et al. Association of Sarcopenia With Short- and Long-term Mortality in Older Adults Admitted to Acute Care Wards: Results From the CRIME Study. The journals of gerontology Series A, Biological sciences and medical sciences. 2014;69(9):1154-61.

10. Mijnarends DM, Meijers JM, Halfens RJ, ter Borg S, Luiking YC, Verlaan S, et al. Validity and reliability of tools to measure muscle mass, strength, and physical performance in community-dwelling older people: a systematic review. Journal of the American Medical Directors Association. 2013;14(3):170-8.

11. Bechtol CO. Grip test; the use of a dynamometer with adjustable handle spacings. The Journal of bone and joint surgery American volume. 1954;36-A(4):820-4; passim.

12. Mathiowetz V, Weber K, Volland G, Kashman N. Reliability and validity of grip and pinch strength evaluations. The Journal of hand surgery. 1984;9(2):222-6.

13. Roberts HC, Denison HJ, Martin HJ, Patel HP, Syddall H, Cooper C, et al. A review of the measurement of grip strength in clinical and epidemiological studies: towards a standardised approach. Age Ageing. 2011;40(4):423-9.

14. Thorngren KG, Werner CO. Normal grip strength. Acta orthopaedica Scandinavica. 1979;50(3):255-9.

15. Desrosiers J, Bravo G, Hebert R, Dutil E. Normative data for grip strength of elderly men and women. The American journal of occupational therapy : official publication of the American Occupational Therapy Association. 1995;49(7):637-44.

16. Helewa $\mathrm{A}$, Goldsmith $\mathrm{CH}$, Smythe $\mathrm{HA}$. The modified sphygmomanometer-an instrument to measure muscle strength: a validation study. Journal of chronic diseases. 1981;34(7):353-61.

17. Culjak M, Skreb-Rakijasic N, Strizrep T. [Measurement of motor strength of the hand using the Martin Vigorometer in evaluating the success of physical therapy in arthritis of the thumb]. Reumatizam. 1993;40(1):21-3.

18. Desrosiers J, Hebert R, Bravo G, Dutil E. Comparison of the Jamar dynamometer and the Martin vigorimeter for grip strength measurements in a healthy elderly population. Scandinavian journal of rehabilitation medicine. 1995;27(3):137-43.

19. Funfgeld EW. [The vigorimeter: for measurement of the strength of the hand and simulation testing]. Deutsche medizinische Wochenschrift (1946). 1966;91(49):2214-6. 
20. Keevil V, Mazzuin Razali R, Chin AV, Jameson K, Aihie Sayer A, Roberts H. Grip strength in a cohort of older medical inpatients in Malaysia: a pilot study to describe the range, determinants and association with length of hospital stay. Archives of gerontology and geriatrics. 2013;56(1):155-9.

21. Roberts HC, Syddall HE, Sparkes J, Ritchie J, Butchart J, Kerr A, et al. Grip strength and its determinants among older people in different healthcare settings. Age and ageing. 2014;43(2):241-6.

22. Bautmans I, Gorus E, Njemini R, Mets T. Handgrip performance in relation to selfperceived fatigue, physical functioning and circulating IL-6 in elderly persons without inflammation. BMC geriatrics. 2007;7:5.

23. Bautmans I, Mets T. A fatigue resistance test for elderly persons based on grip strength: reliability and comparison with healthy young subjects. Aging clinical and experimental research. 2005;17(3):217-22.

24. Bielderman A, van der Schans CP, van Lieshout MR, de Greef MH, Boersma F, Krijnen WP, et al. Multidimensional structure of the Groningen Frailty Indicator in communitydwelling older people. BMC geriatrics. 2013;13:86.

25. Malnutrition Advisory Group BAPEN. Malnutrition Universal Screening Tool. 2008. p. 11,2 .

26. Hoogendijk EO, van Hout HP. Investigating measurement properties of the Groningen Frailty Indicator: a more systematic approach is needed. Journal of the American Medical Directors Association. 2012;13(8):757; author reply -8.

27. Peters LL, Boter H, Buskens E, Slaets JP. Measurement properties of the Groningen Frailty Indicator in home-dwelling and institutionalized elderly people. Journal of the American Medical Directors Association. 2012;13(6):546-51.

28. Nagaratnam N, Gayagay G, Jr. Validation of the Cumulative Illness Rating Scale (CIRS) in hospitalized nonagenarians. Archives of gerontology and geriatrics. 2007;44(1):29-36.

29. Kruizenga HM, Seidell JC, de Vet HC, Wierdsma NJ, van Bokhorst-de van der Schueren MA. Development and validation of a hospital screening tool for malnutrition: the short nutritional assessment questionnaire (SNAQ). Clinical nutrition (Edinburgh, Scotland). 2005;24(1):75-82.

30.

Katz

S, Akpom CA. 12. Index of ADL. Medical care. 1976;14(5 Suppl):116-8.

31. Katz S, Ford AB, Moskowitz RW, Jackson BA, Jaffe MW. Studies of Illness in the Aged. The Index of Adl: A Standardized Measure of Biological and Psychosocial Function. JAMA. 1963;185:914-9.

32. Fisher S, Ottenbacher KJ, Goodwin JS, Graham JE, Ostir GV. Short Physical Performance Battery in hospitalized older adults. Aging clinical and experimental research. 2009;21(6):445-52.

33. Volpato S, Cavalieri M, Sioulis F, Guerra G, Maraldi C, Zuliani G, et al. Predictive value of the Short Physical Performance Battery following hospitalization in older patients. The journals of gerontology Series A, Biological sciences and medical sciences. 2011;66(1):89-96.

34. MacKnight C, Rockwood K. A Hierarchical Assessment of Balance and Mobility. Age and ageing. 1995;24(2):126-30.

35. Hubbard RE, Eeles EM, Rockwood MR, Fallah N, Ross E, Mitnitski A, et al. Assessing balance and mobility to track illness and recovery in older inpatients. Journal of general internal medicine. 2011;26(12):1471-8.

36. Chien MY, Huang TY, Wu YT. Prevalence of sarcopenia estimated using a bioelectrical impedance analysis prediction equation in community-dwelling elderly people in Taiwan. J Am Geriatr Soc. 2008;56(9):1710-5.

37. Folstein MF, Folstein SE, McHugh PR. "Mini-mental state". A practical method for grading the cognitive state of patients for the clinician. Journal of psychiatric research. 1975;12(3):189-98. 
38. Oldfield RC. The assessment and analysis of handedness: the Edinburgh inventory. Neuropsychologia. 1971;9(1):97-113.

39. Lafont C, Gerard S, Voisin T, Pahor M, Vellas B. Reducing "iatrogenic disability" in the hospitalized frail elderly. The journal of nutrition, health \& aging. 2011;15(8):645-60.

40. Bohannon RW, Schaubert KL. Test-retest reliability of grip-strength measures obtained over a 12-week interval from community-dwelling elders. Journal of hand therapy : official journal of the American Society of Hand Therapists. 2005;18(4):426-7, quiz 8.

41. Jones E, Hanly JG, Mooney R, Rand LL, Spurway PM, Eastwood BJ, et al. Strength and function in the normal and rheumatoid hand. The Journal of rheumatology. 1991;18(9):1313-8.

42. Molenaar HM, Zuidam JM, Selles RW, Stam HJ, Hovius SE. Age-specific reliability of two grip-strength dynamometers when used by children. The Journal of bone and joint surgery American volume. 2008;90(5):1053-9.

43. Ewing-Fess E. A method for checking Jamar dynamometer calibration. Journal of hand therapy $1987 ; 1(1): 28-32$.

44. Merkies IS, Schmitz PI, Samijn JP, Meche FG, Toyka KV, van Doorn PA. Assessing grip strength in healthy individuals and patients with immune-mediated polyneuropathies. Muscle \& nerve. 2000;23(9):1393-401. 
Supplementary table 1: Correlations with maximal handgrip strength for both Martin Vigorimeter and Jamar handheld dynamometer

\begin{tabular}{|c|c|c|c|}
\hline & $n$ & Martin Vigorimeter & Jamar dynamometer \\
\hline Age & 96 & -0.195 & -0.180 \\
\hline Height & 91 & $0.438^{* *}$ & $0.517^{\star *}$ \\
\hline Weight & 91 & $0.349^{* *}$ & $0.334^{* *}$ \\
\hline Pain & 40 & -0.116 & -0.289 \\
\hline MMSE & 67 & 0.024 & 0.182 \\
\hline CIRS & 92 & -0.113 & -0.105 \\
\hline GFI & 92 & -0.181 & -0.178 \\
\hline Hand circumference & 92 & $0.459^{* \pi}$ & $0.519^{\text {** }}$ \\
\hline Hand length & 93 & $0.244^{\pi}$ & $0.280^{\pi \pi}$ \\
\hline SMM & 37 & $0.491^{\pi \pi}$ & $0.544^{\pi \pi}$ \\
\hline SMI & 37 & 0.238 & 0,231 \\
\hline RMM & 37 & -0.093 & -0.110 \\
\hline IV needle & 40 & 0.174 & 0.228 \\
\hline Hand problem & 8 & 0.050 & 0.175 \\
\hline SNAQ & 93 & -0.048 & -0.096 \\
\hline Katz ADL-6 & 94 & $-0.362^{\pi \pi}$ & $-0.353^{\pi \times}$ \\
\hline CRP & 85 & 0.073 & 0.154 \\
\hline Creatinine & 81 & 0.075 & 0.108 \\
\hline DOSS & 37 & -0.260 & -0.186 \\
\hline Delirium & 92 & 0.140 & 0.116 \\
\hline SPPB score & 51 & $0.310^{\pi}$ & $0.345^{\pi}$ \\
\hline HABAM score & 45 & $0.432^{\pi \pi}$ & $0.345^{*}$ \\
\hline
\end{tabular}

Data represent Spearman's rho for Maximum strength of the dominant hand measured at T0 for both the Jamar dynamometer and Martin Vigorimeter. Pain: pain during measuring handgrip strength. MMSE: Mini Mental State Examination, CIRS: Cumulative IIIness Rating Scale, GFI: Groningen Frailty Indicator, SMM: Skeletal Muscle Mass, SMI: SMM index (SMM/height squared), RMM: relative muscle mass (SMM/weight), IV needle: IV needle in the dominant hand during measurement, SNAQ: Short Nutritional Assessment Questionnaire, Katz ADL-6: activities of daily living, CRP:C-reactive protein, DOSS: Delirium Observation Scale Score, SPPB: Short Physical Performance Battery, HABAM: Hierarchical Balance and Mobility ${ }^{*} P<0.05$. ${ }^{* *} P<0.001$. 
Supplementary table 2: Subgroup analysis $(n=32)$ with functional parameters and muscle mass as potential predictors for handgrip strength

Martin Vigorimeter
Jamar dynamometer

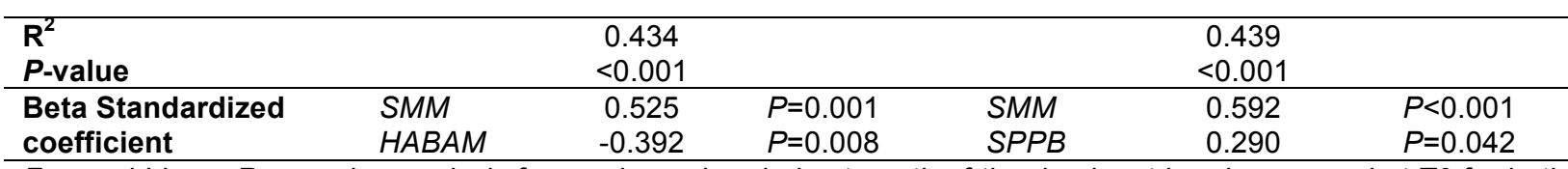

Forward Linear Regression analysis for maximum handgrip strength of the dominant hand measured at T0 for both the Jamar dynamometer and Martin Vigorimeter. Skeletal muscle mass (SMM), Short Physical Performance Battery (SPPB) and Hierarchical balance and mobility (HABAM) were included as potential predictors for handgrip strength. 
Supplementary table 3: Intraclass correlation coefficients (ICC) for maximal handgrip strength for the dominant hand in subgroups based on measuring position, handgrip strength and frailty index.

\begin{tabular}{lcc}
\hline & Jamar dynamometer & Martin Vigorimeter \\
\hline All patients, $\mathrm{n}=96$ & 0.94 & 0.92 \\
Bed-ridden, $\mathrm{n}=28$ & 0.95 & 0.88 \\
Southampton protocol, $\mathrm{n}=68$ & 0.93 & 0.94 \\
Lower strength quartile, $\mathrm{n}=27$ & 0.72 & 0.69 \\
Highest strength quartile, $\mathrm{n}=23$ & 0.91 & 0.70 \\
GFI $\leq 7, \mathrm{n}=56$ & 0.95 & 0.94 \\
GFI >7, $\mathrm{n}=36$ & 0.93 & 0.90
\end{tabular}

Average measures and type A Intraclass Correlation Coefficients (ICC) using an absolute agreement definition was chosen. Frailty was defined according the Groningen Frailty Indicator (GFI) with GFI $\leq 7$ as less frail and GFI>7 as more frail. 


\section{CHAPTER 6}

General discussion 


\section{General discussion}

In this dissertation, we provided more insight into sarcopenia-associated issues within the specific setting of the acute care geriatric ward. We show that sarcopenia is highly prevalent in acutely ill hospitalized geriatric patients (Chapter 2) and has a negative impact on outcome, e.g. mortality (Chapter 3 ) and functional decline $(1,2)$. Geriatricians aim to provide state of the art care for their patients in acute care geriatric wards. A main focus and challenge of geriatric care is to combat functional loss and loss of mobility that is prevalent in nearly all patients. The loss of mobility and functional decline are apparent negative consequences of pre-existing sarcopenia (3).

A basic geriatric principle is that a comprehensive geriatric assessment should be performed upon hospital admission (4). In this context, the attending physician should gather information from the general practitioner and spouse/family carers about the course of functional loss and level of mobility in the pre-hospital phase leading up to admission. This is because the level of premorbid physical function and mobility and the associated changes herein, due to the intercurrent illness, are important in setting treatment targets. In the ideal situation, screening for the presence of sarcopenia should be done as part of the comprehensive geriatric assessment. Therefore, muscle strength and muscle mass should be measured soon after admission (1). In accordance with the EWGSOP-2 consensus on definition and diagnosis statement, muscle strength and functional capacity should be measured by measuring handgrip strength or with the chair stand test (5). For the acute care geriatric ward an adapted diagnostic algorithm for sarcopenia is proposed (Figure 1). This algorithm is adapted from the EWGSOP-2 diagnostic algorithm for sarcopenia (5). Adaptation is necessary because of the specific characteristics of the acutely ill hospitalized geriatric patients, like the presence of low physical capacity and hydration problems.

The chair stand test is preferred as the main strength test, because it is considered to be a key indicator of muscle strength in the lower limbs, muscle performance, has been applied as a predictor of falls and declines in activities of daily living and has a strong correlation with walking speed and is best related to mobility (5-10). However, many acutely ill hospitalized geriatric patients need assistance in rising from a chair. Most patients, who are able to rise independently from a chair, are too weak to perform a 5 times repeated chair stand test.

Therefore, in hospitalized geriatric patients the optimal variant of the chair stand test is a single chair rise with the best time recorded for two attempts (11). In the hospitalized geriatric patients who are not able to rise independently from a chair, handgrip strength represents a feasible and valid alternative (12-14). Handgrip strength has often been proposed as a proxy for overall muscle strength. However, handgrip strength has a moderate correlation with knee extension strength in healthy older adults and is, therefore, probably not a good proxy for 
upper leg strength or ambulation (14). Unfortunately, there is no feasible and more valid alternative for assessing muscle strength in the acute care geriatric ward. Handgrip strength is usually assessed using the Jamar dynamometer. Handgrip strength should preferably be assessed by following the Southampton protocol (Chapter 5). According to this protocol the patient is seated on an arm rested chair (15).

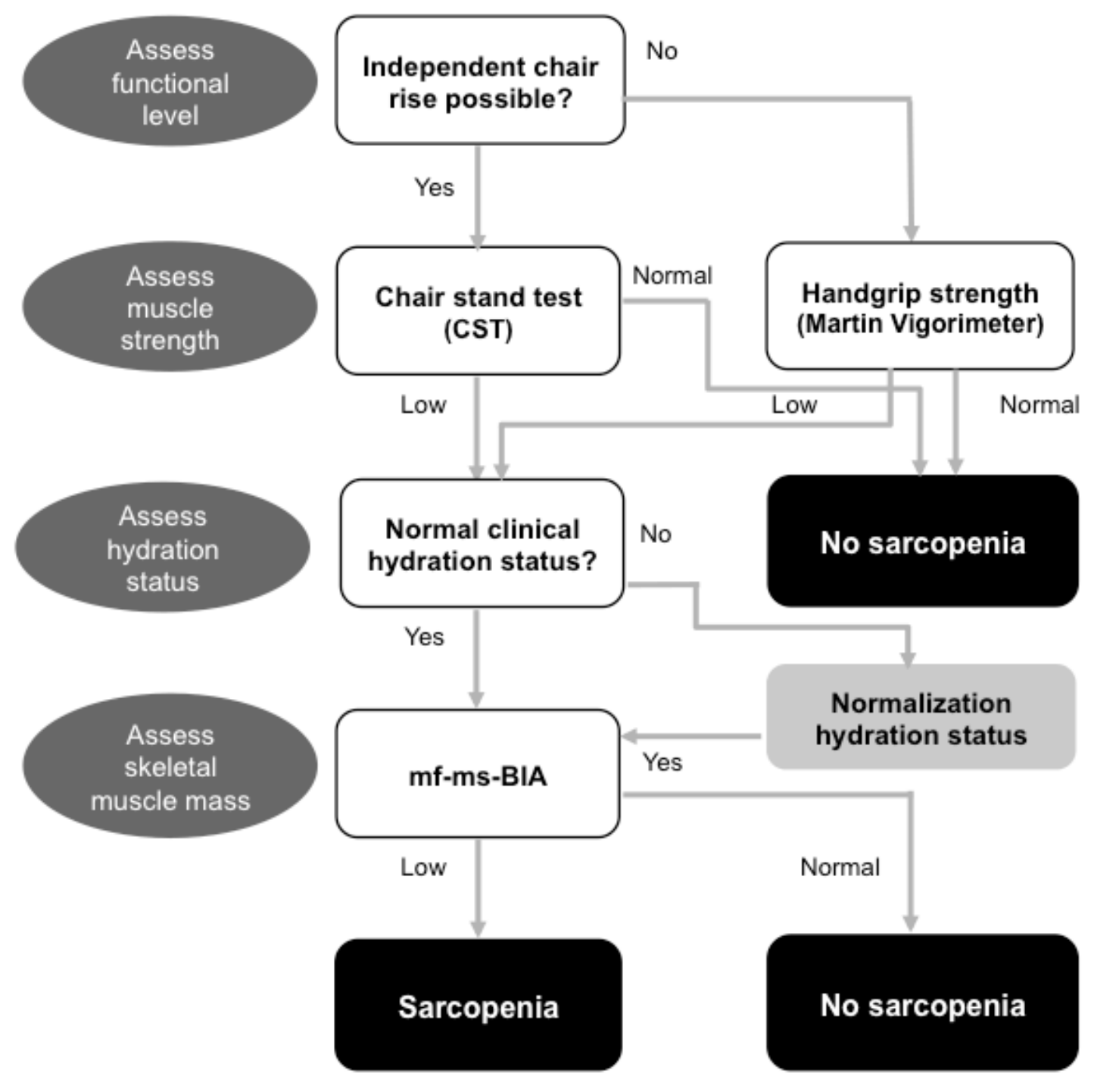

Figure 1: Diagnostic algorithm for sarcopenia in the acute care geriatric ward. mf-ms-BIA: multifrequency-multisegmental Bio-Impedance-Analysis

However, this procedure is often too complicated for hospitalized geriatric patients because of several reasons. First, many geriatric patients frequently have painful arthritic hands, which makes handling of the $1.5 \mathrm{~kg}$ weighing Jamar dynamometer challenging. Second, most geriatric patients have limited mobility and may not be able to make the transfer from the bed 
to an arm rested chair independently and would need assistance from nursing staff (Chapter 5) (15). A feasible and valuable alternative is the use of the Martin Vigorimeter because it has been specifically designed for subjects suffering from arthritis to allow proper grip strength measurement while avoiding excess stress on weak or painful joints $(16,17)$. The assessment of strength with the Martin Vigorimeter can be done with minimal requirements for position and optimal capacity for the patients to generate maximal grip in a (for them) comfortable position. In Chapter 5, we show that handgrip strength measurements with the Martin Vigorimeter are feasible and have good test-retest reliability in the acutely ill geriatric patient. Moreover, the outcomes of these measurements have a good correlation with measurements performed with the Jamar dynamometer (Chapter 5). Therefore, we propose that handgrip strength should be measured with the Martin Vigorimeter in hospitalized acutely ill geriatric patients.

Apart from measuring muscle strength, skeletal muscle mass should be measured soon after hospital admission. MRI, CT and DXA are more or less seen as gold standards to assess muscle mass, however they are generally not practical or easy to perform within the geriatric ward. Bio-impedance analysis (BIA) is much more feasible for bedside application in the acute care geriatric ward, as it does not expose patients to radiation and is relatively inexpensive (18). BIA systems range from single to multiple frequency, and measure whole-body or segmental (upper-lower arm, upper-lower leg, and the trunk) electrical pathways. With multiple-frequencies multi-segmental BIA (mf-ms-BIA) it is possible to measure segmental muscle mass which is important for assessing appendicular and upper leg muscle mass. Additionally mf-ms-BIA is preferred above single frequency BIA because there is less impact of an abnormal hydration status on the reliability of the measurement (18). This is relevant because a disturbed hydration status is frequently present in acutely ill hospitalized patients. Apart from determining appendicular muscle mass as a component of screening for sarcopenia in patients, BIA can also be used to monitor changes in skeletal muscle mass throughout hospitalization and after specific intervention programs aimed at maintaining or even increasing muscle mass. In Chapter 4 , it was demonstrated that mf-ms-BIA is a feasible method to measure skeletal muscle mass in euvolemic hospitalized geriatric patients with a good test-retest reliability. However, if the patient has an abnormal hydration status, measurement of skeletal muscle mass should be done after the hydration status has been compensated (Chapter 4).

\section{Treatment of sarcopenia in the geriatric ward}

The results of the comprehensive geriatric assessment and the assessment of existing sarcopenia are the starting point of a tailor made multidisciplinary treatment strategy taking into account the patient's premorbid level of functioning, treatment preferences, and overall 
prognosis. Treatment of the acute illness should be combined with early mobilization, and accompanied with personalised exercise training (under supervision of the physiotherapist) and nutritional intervention (under supervision of the dietician). This approach should be continued after hospital discharge for at least 3 months (19-23). Therefore, at discharge from the hospital, adequate information must be provided to the general practitioner and both the community physiotherapist as well as the dietician. This should at least include information on the course of the disease, the applied intervention(s), the pre-hospital and current level of functioning, the medication and directions for follow up treatment. After hospital discharge, a tailor made progressive resistance exercise training combined with nutritional intervention should be continued for at least 8 to 12 weeks $(22,24)$. Regular assessments of treatment effects, e.g. once a month, of muscle mass, muscle strength, and level of functioning will be essential in the scope of the personalized treatment targets and to maximize patient adherence and compliance to the program $(22,24)$.

\section{Future research}

In this last paragraph, we suggest directions for future research that can provide insights to improve the clinical outcome of acutely ill hospitalized geriatric patients. First our proposed algorithm for diagnosing sarcopenia should be incorporated in the diagnostic process in the acute care geriatric wards in our country. With this strategy a large database can be generated to give the algorithm more body of evidence in diagnosing sarcopenia in the acutely ill hospitalized geriatric patients. In addition to a proper and feasible diagnostic process at hospital admission, progressive (functional) resistance training should be initiated immediately to prevent further muscle and strength loss and combat sarcopenia. As the geriatric patient population in the acute care geriatric ward is very heterogeneous, resistance training should be personalized as conventional resistance type exercise training is generally impossible. In many cases, these patients are bedridden and alternative strategies to prevent further muscle loss are warranted. Exercise mimetics such as neuromuscular electrical stimulation (NMES) have proven effective in preventing muscle loss during limb immobilisation (25) as well as in comatose patients (26-28). NMES may represent an effective strategy to prevent further muscle loss during the early stages of hospital admission of the acutely ill, geriatric patients. More research is warranted do design practical and effective personalized training strategies and evaluate the capacity to apply exercise mimetics in this patient population.

Energy and protein malnutrition is a major problem in the geriatric patient population. When trying to prevent or treat sarcopenia, adequate nutritional support is required. As the capacity to ingest adequate amounts of food with each main meal is compromised, it may be beneficial to apply an additional pre-sleep meal moment. Previous work has reported that pre-sleep 
protein ingestion is an effective interventional strategy to increase muscle protein synthesis rates during overnight sleep and can be applied to support the skeletal muscle adaptive response to resistance-type exercise training (29). Furthermore, amino acid supplementation may support preservation or gains in muscle mass, strength and/or physical performance in older adults (30-32). Additional work is needed to study the feasibility and effects of pre-sleep protein ingestion and specific amino acid supplementation on the preservation of muscle mass, muscle strength, and physical performance in acutely ill hospitalized sarcopenic geriatric patients.

\section{Mister T's prospects}

Apart from further research to address specific questions concerning the acutely ill geriatric patients mentioned in the previous paragraph, better overall awareness is needed about sarcopenia and its consequences in geriatric medicine, community medicine, as well in hospital care. Everyone, patients, family, general practitioners, nurses, physical therapists, dieticians, health insurance companies, the government, etcetera should be aware of the importance of sarcopenia and the possibility to prevent and combat it. Information about the importance of (physical) healthy ageing, including the role of exercise and nutrition in this process, should be provided to the community. Education programs on the prevention and treatment of sarcopenia are needed for general practitioners and other health care professionals.

What does this ultimately mean for mister T? When the clinical care scenario we prefer and describe above will become mainstream, the case of mister T may turn into a success story:

...... The day after mister T's hospitalization, muscle strength and whole-body and upper leg skeletal muscle mass were assessed, and he was diagnosed as sarcopenic. Sarcopenia was found to be partly primary of origin and partly secondary due to the combination of inflammation, protein malnutrition, and immobility. A tailor made progressive exercise training program with optimization of nutritional status was started on that same day and continued during hospital stay. A few days before hospital discharge, there was a transitional contact between the geriatric multi-disciplinary team and the general practitioner, physiotherapist, and dietician in the community during which appointments were made about the necessary followup care. After discharge to his own home, his mobility was still somewhat limited; although he was able to independently rise from his bed and chair and to dress up by himself, he was still not able to climb the stairs. A bed was temporarily placed in the living room. Tailored physiotherapy and support by the dietician started the day after hospital discharge and were continued at home in close collaboration with the general practitioner. After three weeks he 
was able to climb the stairs again and to sleep in his own bed next to his wife. Three months later, he could take over his original pre hospital life pattern. He now enjoys his retirement and has picked up his hobby gardening. 


\section{References}

1. Cruz-Jentoft AJ, Baeyens JP, Bauer JM, Boirie Y, Cederholm T, Landi F, et al. Sarcopenia: European consensus on definition and diagnosis: Report of the European Working Group on Sarcopenia in Older People. Age Ageing. 2010;39(4):412-23.

2. Cruz-Jentoft AJ, Landi F, Topinkova E, Michel JP. Understanding sarcopenia as a geriatric syndrome. Curr Opin Clin Nutr Metab Care. 2010;13(1):1-7.

3. Welch C, Z KH-S, C AG, J ML, T AJ. Acute Sarcopenia Secondary to Hospitalisation An Emerging Condition Affecting Older Adults. Aging Dis. 2018;9(1):151-64.

4. Ahlund $\mathrm{K}$, Back $\mathrm{M}$, Oberg $\mathrm{B}$, Ekerstad $\mathrm{N}$. Effects of comprehensive geriatric assessment on physical fitness in an acute medical setting for frail elderly patients. Clin Interv Aging. 2017;12:1929-39.

5. Cruz-Jentoft AJ, Bahat G, Bauer J, Boirie Y, Bruyere O, Cederholm T, et al. Sarcopenia: revised European consensus on definition and diagnosis. Age Ageing. 2019;48(1):16-31.

6. Harris-Love MO, Benson K, Leasure E, Adams B, McIntosh V. The Influence of Upper and Lower Extremity Strength on Performance-Based Sarcopenia Assessment Tests. J Funct Morphol Kinesiol. 2018;3(4).

7. Bodilsen AC, Juul-Larsen HG, Petersen J, Beyer N, Andersen O, Bandholm T. Feasibility and inter-rater reliability of physical performance measures in acutely admitted older medical patients. PLoS One. 2015;10(2):e0118248.

8. Bohannon RW. Reference values for the five-repetition sit-to-stand test: a descriptive meta-analysis of data from elders. Percept Mot Skills. 2006;103(1):215-22.

9. Yamako G, Deng G, Totoribe K, Chosa E. A novel protocol to test age-related decreases in sit-to-stand movement abilities in healthy subjects. J Orthop Sci. 2016;21(4):517-23.

10. Yanagawa N, Shimomitsu T, Kawanishi M, Fukunaga T, Kanehisa H. Relationship between performances of 10-time-repeated sit-to-stand and maximal walking tests in non-disabled older women. J Physiol Anthropol. 2016;36(1):2.

11. Mehmet $\mathrm{H}$, Yang AWH, Robinson SR. What is the optimal chair stand test protocol for older adults? A systematic review. Disabil Rehabil. 2019:1-8.

12. Samuel D, Wilson K, Martin HJ, Allen R, Sayer AA, Stokes M. Age-associated changes in hand grip and quadriceps muscle strength ratios in healthy adults. Aging Clin Exp Res. 2012;24(3):245-50.

13. Giampaoli S, Ferrucci L, Cecchi F, Lo Noce C, Poce A, Dima F, et al. Hand-grip strength predicts incident disability in non-disabled older men. Age Ageing. 1999;28(3):283-8.

14. Bohannon RW, Magasi SR, Bubela DJ, Wang YC, Gershon RC. Grip and knee extension muscle strength reflect a common construct among adults. Muscle Nerve. 2012;46(4):555-8.

15. Roberts HC, Denison HJ, Martin HJ, Patel HP, Syddall H, Cooper C, et al. A review of the measurement of grip strength in clinical and epidemiological studies: towards a standardised approach. Age Ageing. 2011;40(4):423-9.

16. Helewa A, Goldsmith $\mathrm{CH}$, Smythe $\mathrm{HA}$. The modified sphygmomanometer-an instrument to measure muscle strength: a validation study. J Chronic Dis. 1981;34(7):353-61.

17. Mathiowetz V, Weber K, Volland G, Kashman N. Reliability and validity of grip and pinch strength evaluations. J Hand Surg Am. 1984;9(2):222-6.

18. Kyle UG, Bosaeus I, De Lorenzo AD, Deurenberg P, Elia M, Manuel Gomez J, et al. Bioelectrical impedance analysis-part II: utilization in clinical practice. Clin Nutr. 2004;23(6):1430-53.

19. Yoshimura $\mathrm{Y}$, Wakabayashi H, Yamada M, Kim H, Harada A, Arai H. Interventions for Treating Sarcopenia: A Systematic Review and Meta-Analysis of Randomized Controlled Studies. J Am Med Dir Assoc. 2017;18(6):553 e1- e16. 
20. Cruz-Jentoft AJ, Landi F, Schneider SM, Zuniga C, Arai H, Boirie Y, et al. Prevalence of and interventions for sarcopenia in ageing adults: a systematic review. Report of the International Sarcopenia Initiative (EWGSOP and IWGS). Age Ageing. 2014;43(6):74859.

21. Beaudart C, McCloskey E, Bruyere O, Cesari M, Rolland Y, Rizzoli R, et al. Sarcopenia in daily practice: assessment and management. BMC Geriatr. 2016;16(1):170.

22. Beaudart C, Dawson A, Shaw SC, Harvey NC, Kanis JA, Binkley N, et al. Nutrition and physical activity in the prevention and treatment of sarcopenia: systematic review. Osteoporos Int. 2017;28(6):1817-33.

23. Deutz NE, Bauer JM, Barazzoni R, Biolo G, Boirie Y, Bosy-Westphal A, et al. Protein intake and exercise for optimal muscle function with aging: recommendations from the ESPEN Expert Group. Clin Nutr. 2014;33(6):929-36.

24. Papa EV, Dong X, Hassan M. Resistance training for activity limitations in older adults with skeletal muscle function deficits: a systematic review. Clin Interv Aging. 2017; 12:955-61.

25. Dirks ML, Wall BT, Snijders T, Ottenbros CL, Verdijk LB, van Loon LJ. Neuromuscular electrical stimulation prevents muscle disuse atrophy during leg immobilization in humans. Acta Physiol (Oxf). 2014;210(3):628-41.

26. Dirks ML, Hansen D, Van Assche A, Dendale P, Van Loon LJ. Neuromuscular electrical stimulation prevents muscle wasting in critically ill comatose patients. Clin Sci (Lond). 2015;128(6):357-65.

27. Herzig D, Maffiuletti NA, Eser P. The Application of Neuromuscular Electrical Stimulation Training in Various Non-neurologic Patient Populations: A Narrative Review. PM R. 2015;7(11):1167-78.

28. Langeard A, Bigot L, Chastan N, Gauthier A. Does neuromuscular electrical stimulation training of the lower limb have functional effects on the elderly?: A systematic review. Exp Gerontol. 2017;91:88-98.

29. Snijders T, Trommelen J, Kouw IWK, Holwerda AM, Verdijk LB, van Loon LJC. The Impact of Pre-sleep Protein Ingestion on the Skeletal Muscle Adaptive Response to Exercise in Humans: An Update. Front Nutr. 2019;6:17.

30. Deutz NE, Pereira SL, Hays NP, Oliver JS, Edens NK, Evans CM, et al. Effect of betahydroxy-beta-methylbutyrate (HMB) on lean body mass during 10 days of bed rest in older adults. Clin Nutr. 2013;32(5):704-12.

31. Cruz-Jentoft AJ. Beta-Hydroxy-Beta-Methyl Butyrate (HMB): From Experimental Data to Clinical Evidence in Sarcopenia. Curr Protein Pept Sci. 2018;19(7):668-72.

32. Oktaviana J, Zanker J, Vogrin S, Duque G. The Effect of beta-hydroxy-betamethylbutyrate (HMB) on Sarcopenia and Functional Frailty in Older Persons: A Systematic Review. J Nutr Health Aging. 2019;23(2):145-50. 


\title{
CHAPTER 7
}

\section{Summary}

\section{Samenvatting}

\author{
Valorisation
}

Dankwoord

Curriculum Vitae

\author{
Publications
}


Summary 


\section{Summary}

The acutely ill hospitalized geriatric patient is usually very frail, and has many co-morbidities and (acute) limitations in his functioning. Despite proactive multidisciplinary treatment during hospital admission aimed at functional recovery, there is still a decline in mobility, physical functioning and self-reliance in a significant proportion of these geriatric patients. In addition to the frailty, co-morbidity and severity of the acute disease, sarcopenia plays a key role in the functional decline. On the other hand, sarcopenia can also develop or worsen, as a consequence of hospitalization, resulting in a vicious circle of deterioration. According to the latest European consensus, sarcopenia is defined as a progressive loss of muscle strength and muscle mass caused by aging itself, as well as by the consequences of diseases associated with inflammation, inactivity and inadequate protein intake. Sarcopenia is associated with an increased risk of developing or worsening frailty, loss of function, loss of self-reliance, complications, loss of quality of life, and an increased risk of premature death. It is important for a treating geriatrician to identify all potentially treatable problems at the earliest possible stage and to place them in the overall context of the geriatric patient. Early recognition of sarcopenia could form the starting point for a targeted treatment to prevent its negative consequences. Every geriatric patient admitted to an acute care geriatric hospital ward is submitted to a comprehensive geriatric assessment, but screening or examination for the presence of sarcopenia is not (yet) part of this. Therefore, little is known about the prevalence and consequences of sarcopenia in this specific group of elderly people. Although there are several ways to diagnose sarcopenia, little is known about the applicability and reliability of these methods in the acutely ill geriatric patient admitted to the hospital. This thesis focuses on the prevalence and negative consequences of sarcopenia, and describes several measurement methods to determine sarcopenia in the acutely ill geriatric patient admitted to the acute care geriatric hospital ward.

Worldwide, there is no consensus (yet) regarding the definition and exact criteria for diagnosing sarcopenia. Current knowledge is mostly based on epidemiological studies in healthy elderly people. The measurement methods and cut-off values used are therefore not readily applicable to acutely ill geriatric hospitalized patients. In chapter 2, we therefore investigated the influence of different diagnostic criteria on the prevalence of sarcopenia in geriatric patients admitted to the acute care geriatric hospital ward. To determine sarcopenia, we measured walking speed (4 meter walk test), handgrip strength (Jamar handheld dynamometer and Martin Vigorimeter) and skeletal muscle mass (bio-impedance analysis, Maltron Bioscan-II). The prevalence of sarcopenia within the acute care geriatric hospital ward was high, but varies greatly depending on the criteria used for sarcopenia, ranging between 
$26-75 \%$ in women and $42-100 \%$ in men. The prevalence of sarcopenia was also highly dependent on the measurement method and cut-off value used. For example, the prevalence was different with a different formula used (Janssen vs Maltron) to measure skeletal muscle mass using bio-impedance analysis.

In chapter 3 we investigated whether, as in the general population, there is an association between sarcopenia and adverse outcomes in acutely ill geriatric patients admitted to an acute care geriatric hospital ward. We investigated the relationship and risk between different consensus criteria for sarcopenia and other patient characteristics on one hand, and the risk of death within 1 and 2 years after hospitalization on the other hand. Geriatric patients with sarcopenia had an increased mortality risk within (1 and) 2 years according to the criteria of the European Working Group on Sarcopenia in Older People (EWGSOP) and the Foundation for the National Institutes of Health $(\mathrm{FNIH})$ compared to geriatric patients who did not have sarcopenia. Furthermore, it was found that geriatric patients with a higher fat mass index (fat mass $(\mathrm{kg}) /$ height $^{2}$ ) and a higher phase angle measured by bio-impedance analysis had a better 2-year survival.

To diagnose sarcopenia, muscle mass can be measured by bio-impedance analysis and muscle strength by determining the handgrip strength. These methods have been chosen because they are practical for bedside use in acutely ill hospitalized geriatric patients. Yet, these measurements and their interpretation can be further complicated in acutely ill geriatric patients, because they often have very limited physical and mental capacities and the clinical condition changes during hospital admission. As a result, the choice of the measurement moment during hospital admission can influence the result and, thus the reliability and validity of the measurement. For example, the measurement of skeletal muscle mass can be affected by an abnormal and changing hydration status, disease course and applied treatments. We used multiple frequency bio-impedance analysis (mf-BIA) because this method has been suggested to be able to distinguish between intracellular and extracellular water. As a result, the measurement of muscle mass would be less affected by the presence of extracellular water, such as, for example, with edema. However, no information is available regarding reproducibility and the influence of abnormal hydration status in acutely ill hospitalized geriatric patients. In chapter 4 we therefore looked at the reproducibility of the measurement of skeletal muscle mass by mf-BIA in the first week of hospitalization in acutely ill geriatric patients. During the first week of hospitalization, skeletal muscle mass was measured on 3 days (on day $2 \pm 1,3 \pm 1$ and $5 \pm 2$ after admission). It was determined that the skeletal muscle mass measurement by mf-BIA is a reliable and reproducible method. However, the reliability of the measurement was influenced by an abnormal hydration status of the patient. Based on these 
findings, it is recommended not to measure muscle mass until the patient has reached normal hydration status.

The measurement of handgrip strength is usually done with the Jamar handheld dynamometer, as it is regarded as the gold standard. However, the use of the Jamar handheld dynamometer can be difficult in acutely ill geriatric patients due to the presence of physical limitations. The Martin Vigorimeter could be a good alternative because it has been specifically developed for patients with physical limitations. In chapter $\mathbf{5}$ we investigated the applicability and test-retest reliability of both the Jamar handheld dynamometer and the Martin Vigorimeter in acutely ill geriatric patients admitted to an acute care geriatric hospital ward. The test-retest reliability was found to be good for both instruments. Moreover, there was a strong correlation between both measurement methods. However, the handgrip strength measurement using the Martin Vigorimeter proved to be a more practical method, as mobilization from bed to chair, usually with the help of a nurse, was not necessary.

The overall discussion and practical application of the results from the studies of this dissertation are described in chapter 6 . We conclude that sarcopenia is a common condition in acutely ill hospitalized geriatric patients. We have shown that the presence of sarcopenia in these acutely ill frail patients with co-morbidity and disability is an independent predictor of adverse outcome, such as premature death. Because of the high prevalence and the prognostic significance of sarcopenia, it is therefore recommended to test for sarcopenia upon hospital admission, when performing the comprehensive geriatric assessment. Given the specific characteristics of the geriatric patient, a diagnostic algorithm is proposed that takes into account the heterogeneity of the acutely ill geriatric population, the frequent presence of hydration problems and the often limited physical capabilities. It is proposed to implement this diagnostic algorithm in all geriatric hospital wards in the Netherlands. In this way, a database can be generated that strengthens the evidence that this diagnostic algorithm can represent a valid instrument with good predictive properties. By means of a clinically applicable valid diagnostic algorithm, tailor made exercise training programs and nutritional interventions can be tested for applicability and effectiveness. These steps can contribute to a better physical functioning and better quality of life of the acutely ill geriatric patient admitted to the acute care geriatric hospital ward. 
Samenvatting 


\section{Samenvatting}

De acuut zieke geriatrische patiënt die in het ziekenhuis wordt opgenomen is meestal erg kwetsbaar en heeft veel co-morbiditeiten en (acute) beperkingen in zijn functioneren. Ondanks een pro-actieve multidisciplinaire behandeling tijdens de opname die gericht is op (functieherstel, zien we bij een aanzienlijk deel van de geriatrische patiënten een achteruitgang van de mobiliteit, het fysiek functioneren en de zelfredzaamheid. Naast de kwetsbaarheid, comorbiditeit en de ernst van de acute ziekte, lijkt sarcopenie de functionele achteruitgang voor een belangrijk deel te verklaren. Anderzijds kan sarcopenie deels ook een gevolg zijn van de ziekenhuisopname, resulterend in een vicieuze cirkel van achteruitgang. Sarcopenie wordt volgens de laatste Europese consensus gedefinieerd als een progressief verlies van spierkracht en spiermassa veroorzaakt door zowel veroudering zelf, als ook door de gevolgen van ziekten die gepaard gaan met inflammatie, inactiviteit en een inadequate eiwitinname. Sarcopenie is geassocieerd met een verhoogd risico op het ontstaan of de verergering van kwetsbaarheid, functieverlies, verlies van zelfredzaamheid, complicaties, afname van kwaliteit van leven en ook met een verhoogd risico op voortijdig overlijden. Voor een behandelend geriater is het belangrijk om alle potentieel behandelbare problemen in een zo vroeg mogelijk stadium vast te stellen en te plaatsen in de totale context van de geriatrische patiënt. Een tijdige herkenning van sarcopenie zou hierbij het startpunt kunnen vormen voor een gerichte behandeling ter preventie van de negatieve gevolgen ervan. Bij elke geriatrische patiënt die opgenomen wordt op een geriatrie afdeling van het ziekenhuis vindt een volledig geriatrisch onderzoek plaats, echter structureel onderzoek naar sarcopenie vormt hier (nog) geen onderdeel van. Derhalve is weinig bekend over het vóórkomen en de gevolgen van sarcopenie bij deze specifieke groep ouderen. Ondanks dat er verschillende manieren bestaan om sarcopenie vast te stellen, is er weinig bekend over de toepasbaarheid en betrouwbaarheid van deze methoden bij de acuut zieke geriatrische patiënt, opgenomen in het ziekenhuis. Dit proefschrift beschrijft naast het vóórkomen en de negatieve gevolgen van sarcopenie, enkele meetmethoden om sarcopenie vast te kunnen stellen bij de acuut zieke geriatrische patiënt die opgenomen wordt op een afdeling geriatrie in het ziekenhuis.

Wereldwijd bestaat er (nog) geen consensus met betrekking tot de definitie en de exacte criteria voor de diagnosestelling van sarcopenie. De meeste kennis aangaande het vóórkomen van sarcopenie komt uit epidemiologische studies bij veelal gezonde ouderen. De gehanteerde meetmethoden en afkapwaarden zijn hierdoor niet zonder meer toepasbaar bij in het ziekenhuis opgenomen acuut zieke geriatrische patiënten. In hoofdstuk 2 hebben we onderzocht wat de invloed is van verschillende diagnostische criteria op de prevalentie van sarcopenie bij geriatrische patiënten die opgenomen zijn op de afdeling geriatrie van een 
ziekenhuis. Om sarcopenie vast te stellen hebben we loopsnelheid (4 meter looptest), handknijpkracht (Jamar handheld dynamometer en Martin Vigorimeter) en skeletspiermassa (bio-impedantie analyse, Maltron Bioscan-II) gemeten. De prevalentie van sarcopenie binnen de afdeling geriatrie was hoog, maar was sterk afhankelijk van de gehanteerde criteria voor sarcopenie en varieerde tussen de $26-75 \%$ bij vrouwen en tussen de $42-100 \%$ bij mannen. Ook was de prevalentie van sarcopenie sterk afhankelijk van de gehanteerde meetmethode en afkapwaarde, zoals bijvoorbeeld de gehanteerde formule (Janssen vs Maltron) om de skeletspiermassa te meten met behulp van bio-impedantie.

In hoofdstuk 3 hebben we onderzocht of er, net zoals in de algemene bevolking, een associatie bestaat tussen sarcopenie en negatieve uitkomsten bij de acuut zieke geriatrische patiënt, opgenomen op een afdeling geriatrie. We hebben onderzocht wat de relatie en het risico is tussen enerzijds verschillende consensus criteria voor sarcopenie en overige patiëntkenmerken en anderzijds het risico op overlijden binnen 1 en 2 jaar na de ziekenhuis opname. Aangetoond werd dat geriatrische patiënten met sarcopenie volgens de criteria van de European Working Group on Sarcopenia in Older People (EWGSOP) en de Foundation for the National Institutes of Health (FNIH) een sterk verhoogd risico hadden om binnen (1 en) 2 jaar te overlijden vergeleken met geriatrische patiënten die geen sarcopenie hadden. Bovendien werd vastgesteld dat geriatrische patiënten met een hogere vetmassa index (vetmassa $(\mathrm{kg}) /$ lengte $^{2}$ ) en een hogere fasehoek (phase angle) gemeten met behulp van bioimpedantie analyse een betere 2 jaars overleving hadden.

Om de diagnose sarcopenie vast te stellen kan de spiermassa gemeten worden via bioimpedantie analyse en de spierkracht door het bepalen van de handknijpkracht. Deze methoden zijn gekozen omdat deze praktisch toepasbaar zijn aan bed bij acuut zieke geriatrische patiënten. Deze metingen en de interpretatie daarvan kunnen echter bij acuut zieke geriatrische patiënten extra gecompliceerd zijn, omdat ze vaak fysiek en mentaal erg beperkt belastbaar zijn en de klinische toestand tijdens de opname verandert. Hierdoor kan de keuze van het meetmoment tijdens de opname van invloed zijn op het resultaat en de betrouwbaarheid hiervan. Zo kan de meting van de skeletspiermassa beïnvloed worden door een afwijkende en veranderende hydratietoestand als gevolg van de ziekte en toegepaste behandelingen. We hebben gebruik gemaakt van multipele frequentie bio-impedantie analyse (mf-BIA) omdat van deze methode is gesuggereerd dat deze onderscheid kan maken tussen intracellulair en extracellulair water. Hierdoor zou de meting van de spiermassa minder beïnvloed worden door aanwezigheid van extracellulair water, zoals bijvoorbeeld bij oedeem. $\mathrm{Er}$ is echter geen informatie beschikbaar met betrekking tot de reproduceerbaarheid en de invloed van een afwijkende hydratietoestand bij in het ziekenhuis opgenomen zieke 
geriatrische patiënten. In hoofdstuk 4 hebben we daarom gekeken naar de reproduceerbaarheid van de meting van de skeletspiermassa middels mf-BIA in de eerste week van de ziekenhuisopname bij acuut zieke geriatrische patiënten. Tijdens de eerste week van de ziekenhuis opname werd op 3 dagen de skeletspiermassa gemeten (op dag $2 \pm 1,3 \pm 1$ en $5 \pm 2$ na opname). Hierbij werd vastgesteld dat de skeletspiermassa meting middels mf-BIA een betrouwbare en reproduceerbare methode is. Echter de betrouwbaarheid van de meting werd beïnvloed door een afwijkende hydratie toestand van de patiënt. Op basis van deze bevindingen wordt geadviseerd om de spiermassa pas te meten wanneer de patiënt een normale hydratietoestand heeft bereikt.

De meting van de handknijpkracht wordt meestal gedaan met de Jamar handheld dynamometer, omdat deze wordt gezien als gouden standaard. Het gebruik van de Jamar handheld dynamometer kan echter bij acuut zieke geriatrische patiënten moeilijk zijn door de aanwezige fysieke beperkingen. De Martin Vigorimeter zou een goed alternatief kunnen zijn omdat deze speciaal ontwikkeld is voor patiënten met fysieke beperkingen. In hoofdstuk $\mathbf{5}$ hebben we de toepasbaarheid en de test-hertest betrouwbaarheid onderzocht van zowel de Jamar handheld dynamometer als de Martin Vigorimeter bij acuut zieke geriatrische patiënten, die opgenomen zijn op een afdeling geriatrie van het ziekenhuis. De test-hertest betrouwbaarheid bleek voor beide instrumenten vergelijkbaar goed. Bovendien was er een sterke correlatie tussen beide meetmethoden. Echter, de handknijpkrachtmeting met behulp van de Martin Vigorimeter bleek een meer praktische methode, doordat mobilisatie van bed naar een stoel, meestal met behulp van een verpleegkundige, niet noodzakelijk was.

De algehele discussie en praktische toepassing van de resultaten uit de studies van dit proefschrift worden beschreven in hoofdstuk 6. We concluderen dat sarcopenie een aandoening is die vaak voorkomt bij acuut zieke geriatrische patiënten opgenomen in het ziekenhuis. We hebben aangetoond dat de aanwezigheid van sarcopenie bij deze acuut zieke kwetsbare patiënten met nogal wat co-morbiditeit en beperkingen een onafhankelijke risicofactor vormt voor een slechte uitkomst, zoals voortijdig overlijden. In verband met de hoge prevalentie en de prognostische betekenis van sarcopenie wordt derhalve voor de dagelijkse praktijk aanbevolen om bij opname, bij het uitvoeren van het volledig geriatrisch onderzoek, ook vast te stellen of er sprake is van sarcopenie. Gelet op de specifieke kenmerken van de geriatrische patiënt wordt een diagnostisch algoritme voorgesteld, dat onder andere rekening houdt met de heterogeniteit van de acuut zieke geriatrische populatie, de frequente aanwezigheid van hydratieproblemen en de vaak beperkte fysieke vermogens. Voorgesteld wordt om dit diagnostisch algoritme te implementeren op alle geriatrische afdelingen in Nederland. Op deze manier kan een database gegenereerd worden waarmee 
de bewijskracht wordt versterkt dat dit diagnostisch algoritme een valide instrument kan vormen met goede predictieve eigenschappen. Met een klinisch toepasbaar valide diagnostisch algoritme kunnen bewegings- en voedingsinterventies op maat getoetst worden op toepasbaarheid en effectiviteit. Deze stappen kunnen een bijdrage leveren aan een beter fysiek functioneren en betere kwaliteit van leven van de zieke geriatrische patiënt opgenomen op een geriatrische afdeling van het ziekenhuis. 


\section{Valorisation}




\section{Valorisation}

\section{Relevance}

Both globally and in the Netherlands, we are faced with an ever-aging society. In 2018, there were 3.2 million people aged 65 years and older in the Netherlands, representing $18 \%$ of the population. By 2041 , this number will have grown to 4.8 million people, representing about 27 percent of the total Dutch population (1). Although in 2040, people aged 65 years still have a life expectancy of 22-24 y, the last 15-17 years of their life, they have to deal with physical and functional decline including mobility problems. About $22 \%$ of the people aged $65 \mathrm{y}$ and older are frail and are at risk of functional decline. These frail older adults have more health problems and need more health care, including hospital care, compared to non-frail older adults (2). Within the older population, geriatric patients are the frailest subgroup, usually displaying several co-morbid diseases and physical and/or mental disabilities. When affected by an acute illness, they have an atypical disease presentation with rapid functional decline, and hospitalization is often unavoidable. In 2019, more than 16,000 geriatric patients were admitted to acute care geriatric hospital wards in the Netherlands because of acute problems (3). Therefore, many geriatricians recognize in a significant proportion of acutely hospitalized geriatric patients the case study of Mr. T, which we presented in this thesis. He showed considerable functional decline during hospital admission despite proactive multidisciplinary treatment aimed at functional recovery. In addition to the frailty, co-morbidity and severity of the acute disease, sarcopenia appears to explain this functional decline. On the other hand, sarcopenia can also partly be a consequence of hospitalization itself, resulting in a vicious circle of deterioration. In this dissertation, we conclude that sarcopenia is a common condition in acutely ill hospitalized geriatric patients (chapter 2). We have shown that the presence of sarcopenia in these acutely ill frail patients with co-morbidity and disability is an independent predictor of adverse outcomes including mortality (chapter 3). In addition, we have demonstrated that the assessment of sarcopenia by means of measuring skeletal muscle mass (chapter 4) and muscle strength (chapter 5) needs special attention because of the limited physical capabilities of these patients and the frequent presence of problems with their hydration status. Since all of the data were collected in a real-life hospital setting, many of the findings can be directly translated to daily practice. In doing so we are able to develop tailormade treatment plans to combat functional decline and improve quality of life and reduce health care consumption and costs.

\section{Recommendations}

Because of the high prevalence and the prognostic significance of sarcopenia in acutely hospitalized geriatric patients, it is recommended to determine whether sarcopenia is present 
upon hospital admission. This should be done when performing the comprehensive geriatric assessment (CGA) (4). Given the specific characteristics of the geriatric patient, in this thesis a diagnostic algorithm is proposed that takes into account the heterogeneity of the acutely ill geriatric population, the frequent presence of hydration problems and the often limited physical capabilities (chapter 6). It is strongly recommended to adopt this diagnostic algorithm in all geriatric hospital wards in the Netherlands. In this way, a database can be generated that strengthens the evidence that this diagnostic algorithm can represent a valid instrument with good predictive properties. This can be achieved by creating awareness among geriatricians and residents about the best evidence until now about sarcopenia in the acutely ill hospitalized geriatric patients. This can be done by sharing the results of this thesis to all members and residents of the Dutch Geriatrics Society (5) by providing each member of personal (digital) version. Furthermore, the topic of sarcopenia, and sarcopenia assessment, should be included in the research agenda of the Dutch Geriatrics Society. By means of the existing research network, many Dutch hospitals can participate in the validation of the diagnostic algorithm and become familiar in assessing sarcopenia. After further validation, the diagnostic algorithm should be incorporated in the guideline CGA of the Dutch Geriatrics Society. The author of this thesis has already provided up to date information about sarcopenia in the hospitalized geriatric patient during the input round on the way to the revision of the CGA. In addition, to improve knowledge about sarcopenia in residents in geriatric medicine the author of this thesis, is involved in their training as a regular part of the national education program.

In addition to creating awareness and up to date knowledge among the geriatricians and residents, the acute care geriatric hospital wards will have to be provided with the appropriate equipment. In order to be able to apply the diagnostic algorithm, in addition to a stopwatch, a Martin Vigorimeter and a bio-impedance device are required. The advice is to strive for the same device for all geriatric departments in the Netherlands. The bio-impedance device should be able to measure several body segments separately with different frequencies, which is also relevant for evaluation of treatment plans. Of course, a short training program in the use of these devices should accompany the application in daily practice so that it can be more easily ingrained in the daily departmental routines.

By means of a clinically applicable, valid and feasible diagnostic algorithm, we may be better able to not only diagnose sarcopenia upon hospital admission, but also test tailor-made exercise training programs, nutritional interventions and exercise mimicking treatment options for their applicability and effectiveness, both in daily practice and in research. Apart from such hospital-based applications, it is evident that geriatric patients often come into contact with several care providers, even before hospital admission as well as after hospital discharge. In preventing sarcopenia as early as possible before hospital admission, several steps can be 
taken by the general practitioners. Screening for sarcopenia in patients who visit the general practitioner for something else, or in the elderly patients who come with mobility problems should be embedded in the clinical routine, so that appropriate diagnostics and treatment can start in collaboration with the dietician and physiotherapist. Subsequently, a proper medical transfer between general practitioner and hospital is necessary when referring patients. In doing so, the chain of health care should be aimed at limiting functional decline and keeping these geriatric patients less dependent and able to stay at their homes as long as possible. This is in accordance with government policy.

The results of this thesis urge to create a better overall awareness about sarcopenia and its consequences in geriatric medicine, community medicine, as well in hospital care. The Dutch Geriatrics Society can take a leading role in creating this awareness. As we live in an ageing society with a health policy based on "aging in place", prevention of functional decline is a key topic. Many older (frail) adults are in contact to one or more health care professionals or organisations, at the same time or consecutively. Everyone, including amongst others patients, family, general practitioners, geriatricians, nurses, physical therapists, dieticians and health insurance companies should therefore be informed to become aware of the importance of sarcopenia and the possibility to prevent and combat it. This can be achieved by means of education programs on the prevention, diagnosis and treatment of sarcopenia that should become available and implemented for all these actors. To this end, the results of the studies in this thesis are not only disseminated via the literature and presented on congresses; they will also be used in the development and implementation of education programs as to aid in the overall goal of successful ageing for all individuals in our society. 


\section{References}

1. CBS Statline: Bevolkingsopbouw Nederland. https://opendata.cbs.nl/statline. Updated 2019.

2. Vektis:Ouderenzorg. https://www.vektis.nl. Updated 2019.

3. Nederlandse Zorgautoriteit. https://www.opendisdata.nl. Updated 2020.

4. NVKG. Comprehensive Geriatric

Assessment.https://richtlijnendatabase.nl/richtlijn/comprehensive_geriatric_assessment_cga. Updated 2010.

5. Nederlandse Vereniging voor Klinische Geriatrie. https://www.nvkg.nl/professionals. Updated 2020. 
Dankwoord 


\section{Dankwoord}

Dit onderzoek was onmogelijk geweest zonder de deelname van de ouderen die opgenomen geweest zijn op de afdeling Ouderengeneeskunde van Zuyderland Medisch Centrum. Nu, in 2020, zijn de meesten van hen reeds overleden. Hun bijdrage heeft een waardevol inzicht gegeven in de gevolgen van sarcopenie en waar we rekening mee moeten houden bij het meten van determinanten van sarcopenie.

Met mijn promotieteam bestaande uit prof. Luc van Loon, prof. Jos Schols en dr. Lex Verdijk had ik het niet beter kunnen treffen.

Beste Luc, je was gedurende het hele traject degene die me geleerd heeft om onderscheid te maken tussen klinisch en wetenschappelijk denken. Jouw nuchtere manier van communiceren en uitstekend analytisch denk vermogen waren voor mij erg leerzaam en inspirerend. Mijn bezoekjes aan jou gaven mij telkens weer energie en stof tot nadenken.

Beste Jos, jij was degene die me aanmoedigde om dit traject aan te gaan. Jij hebt voor mij de juiste mensen bij elkaar gebracht. Voor mij was jij een motivator, een procesbewaker, mental coach en een "pater familias". Wat heb jij veel energie, volgens mij sta jij altijd aan! Een reactie op een mail, app of manuscript kwam altijd in een mum van tijd.

Beste Lex, ik heb veel bewondering voor je nauwgezette begeleiding. Jij was mijn directe begeleider en ik heb buitengewoon veel van je geleerd. Dank voor je zorgvuldige beoordeling van alle artikelen die we geschreven hebben.

Beste heren, ik hoop dat we ook na dit traject elkaar blijven ontmoeten en samen verdere stappen kunnen zetten in de verbinding tussen wetenschap en de klinische praktijk.

Rian Bibo, Wout de Blois en Jesper Dörge wil ik graag bedanken voor hun hulp bij het verzamelen van de data. Zonder jullie hulp was het onmogelijk geweest om de metingen bij de patiënten uit te voeren.

De verpleegkundigen van de polikliniek Ouderengeneeskunde van Zuyderland Medisch Centrum hebben de afgelopen jaren regelmatig een bijdrage geleverd aan de uitvoering van metingen. De fysiotherapeuten verbonden aan afdeling Ouderengeneeskunde West 52 hebben de afgelopen jaren geholpen met een deel van de metingen. Ons secretariaat heeft ervoor gezorgd dat een en ander goed gepland werd. Dank voor jullie bereidheid en hulp. Mijn collegae binnen Co-MIK en de vakgroep Ouderengeneeskunde dank voor de ruimte die jullie mij hebben gegeven.

Prof. dr. R.A. de Bie, prof. dr. A.F. Lenssen, prof. dr., C.P.G.M. de Groot, prof. dr. A.M.W.J Schols en prof. dr. M.G.M. Olde Rikkert wil ik hartelijk bedanken voor de inhoudelijke beoordeling van mijn manuscript. 
Lieve familie en vrienden, hartelijk dank voor de belangstelling en steun de afgelopen jaren. Ma en (in herinnering) pa, bedankt voor de bagage die jullie meegaven met een (gezonde) dosis ambitie, een nuchter boeren verstand en een sterk doorzettingsvermogen, dat heb ik goed kunnen gebruiken bij dit promotie traject.

Mijn lieve vrouw Ans, dank voor je onvoorwaardelijke steun en ruimte die je me hebt gegeven. Ans en Simone dank voor jullie hulp bij de metingen en de database. Ans, mijn liefste en mijn maatje, mijn lieve kinderen Yvette, Simone, Michel en Annette wat ben ik dankbaar en gelukkig dat ik het leven met jullie mag delen. 


\section{Curriculum Vitae}




\section{Curriculum Vitae}

Walther Sipers was born on March 13, 1965 in Maastricht, the Netherlands. A farmer's son he grew up with two sisters and two brothers in Margraten, in Limburg. In 1984, he graduated from secondary school, Sophianum in Gulpen, the Netherlands. He studied medicine at the Radboud University of Nijmegen and obtained his medical degree (cum laude) in 1990. Before he started his training in geriatric medicine he worked at a department of geronto-psychiatry in "GGNet", a mental hospital in Apeldoorn, thereafter at the department of neurology in the Canisius Wilhelmina Ziekenhuis in Nijmegen and at the rehabilitation medicine in the Sint Maartenskliniek in Nijmegen. Next, in 1993 he started with a 2-year residency in internal medicine at the Canisius Wilhemina Ziekenhuis in Nijmegen, followed by 2-year geriatric medicine at the Rijnstate Ziekenhuis in Arnhem. His training was completed in 1998 with a 9 month-training in old age psychiatry in the Vincent $v$ Gogh Institute in Venray. During the last year of his medical study and during his traineeship he married and became father of three daughters and one son. He started as the first geriatrician, from 1998 until 2004, in the Deventer hospital. Then he proceeded to work as a geriatrician in Zuyderland Medical Centre until now, thus returning to the southern part of Limburg. From 2015 onwards he works as a trainer in geriatric medicine. The first preparations for the research presented in this thesis were made in 2013. 


\section{Publications}




\section{Publications}

- Ruisch JE, Sipers W, Plum PF, Spaetgens B. Individualized approach to reconsider perioperative do-not-resuscitate orders in frail older patients. Geriatr Gerontol Int. 2020;20(10):989-990. doi: 10.1111/ggi.14030 [doi].

- Castro E, Korver F, Merry A, van Moorsel F, Hazebroek M, Smid M, Ploux S, Bordachar P, Prinzen F, Sipers W, Strik M. Should we still monitor QTc duration in frail older patients on low-dose haloperidol? A prospective observational cohort study. Age Ageing. 2020;49(5):829-836. doi: 10.1093/ageing/afaa066 [doi].

- Twist van DJL, Mostard GJM, Sipers WMWH. Delayed recovery from initial orthostatic hypotension: an expression of frailty in the elderly. Clin Auton Res. 2020 Apr;30(2):105-106. doi: 10.1007/s10286-019-00664-2.

- Sipers, W M W H, Dorge J, Schols, J M G A, Verdijk LB, van Loon, L J C. Multifrequency bioelectrical impedance analysis may represent a reproducible and practical tool to assess skeletal muscle mass in euvolemic acutely ill hospitalized geriatric patients. Eur Geriatr Med. 2020;11(1):155-162. doi: 10.1007/s41999-01900253-6 [doi].

- Sipers, W M W H, de Blois W, Schols, J M G A, van Loon, L J C, Verdijk LB. Sarcopenia is related to mortality in the acutely hospitalized geriatric patient. $J$ Nutr Health Aging. 2019;23(2):128-137. doi: 10.1007/s12603-018-1134-1 [doi].

- Visser M, Schaap LA, Hobbelen J, Perkisas S, Sipers W. Sarcopenie: Screening en diagnose. Ned Tijdschr Geneeskd. 2019;163:D3824:1-7.

- Sipers WM, Verdijk LB, Sipers SJ, Schols JM, van Loon LJ. The martin vigorimeter represents a reliable and more practical tool than the jamar dynamometer to assess handgrip strength in the geriatric patient. J Am Med Dir Assoc. 2016;17(5):466.e1466.e7. doi: 10.1016/j.jamda.2016.02.026 [doi].

- de Wit HA, Hurkens KP, Mestres Gonzalvo C, Smid M, Sipers W, Winkens B, Mulder WJ, Janknegt R, Verhey FR, van der Kuy PH, Schols JM. The support of medication reviews in hospitalised patients using a clinical decision support system. Springerplus. 2016;5(1):871-1. eCollection 2016. doi: 10.1186/s40064-016-2376-1 [doi].

- Vermeulen J, Neyens JC, Spreeuwenberg MD, van Rossum E, Boessen AB, Sipers $\mathbf{W}$, de Witte LP. The relationship between balance measured with a modified bathroom scale and falls and disability in older adults: A 6-month follow-up study. J Med Internet Res. 2015;17(5):e131. doi: 10.2196/jmir.3802 [doi].

- Metzelthin SF, van Rossum E, Hendriks MR, De Witte LP, Hobma SO, Sipers W, Kempen GI. Reducing disability in community-dwelling frail older people: Cost- 
effectiveness study alongside a cluster randomised controlled trial. Age Ageing. 2015;44(3):390-396. doi: 10.1093/ageing/afu200 [doi].

- Sipers WM, Meijers JM, van Dijk RB, Halfens RJ, Schols JM. Impact of different diagnostic criteria on the prevalence of sarcopenia in an acute care geriatric ward. $J$ Frailty Aging. 2014;3(4):222-229. doi: 10.14283/jfa.2014.28 [doi].

- Olde Rikkert MG, Verhey FR, Sijben JW, Bouwman FH, Dautzenberg PL, Lansink M, Sipers WM, van Asselt DZ, van Hees AM, Stevens M, Vellas B, Scheltens P.et al. Differences in nutritional status between very mild alzheimer's disease patients and healthy controls. J Alzheimers Dis. 2014;41(1):261-271. doi: 10.3233/JAD-131892 [doi].

- Sipers WM, Mijnarends DM, Schols JM. Sarcopenia. Ned Tijdschr Tandheelkd. 2013;120(5):236-239. doi: 10.5177/ntvt.2013.05.12233 [doi].

- Metzelthin SF, van Rossum E, de Witte LP, Ambergen AW, Hobma SO, Sipers W, Kempen GI. Effectiveness of interdisciplinary primary care approach to reduce disability in community dwelling frail older people: Cluster randomised controlled trial. BMJ. 2013;347:f5264. doi: 10.1136/bmj.f5264 [doi].

- Vermeulen J, Neyens JC, Spreeuwenberg MD, van Rossum E, Sipers W, Habets H, Hewson DJ, de Witte LP. User-centered development and testing of a monitoring system that provides feedback regarding physical functioning to elderly people. Patient Prefer Adherence. 2013;7:843-854. doi: 10.2147/PPA.S45897 [doi].

- van Asselt DZ, van Bokhorst-de van der Schueren, M A, van der Cammen, T J, Disselhorst LG, Janse A, Lonterman-Monasch S, Maas HA, Popescu ME, SchölzelDorenbos CJ, Sipers WM, Veldhoven CM, Wijnen HH, Olde Rikkert MG. Assessment and treatment of malnutrition in dutch geriatric practice: Consensus through a modified delphi study. Age Ageing. 2012;41(3):399-404. doi: 10.1093/ageing/afs005 [doi].

- Sipers, W. M. W. H. Sarcopenie en andere vormen van functieverlies. In: J.W.M. Muris, C. M. E. E. de Weerd Spaetgens, ed. Ouderengeneeskunde. Bohn Stafleu van Loghum; 2012:91-107.

- Daniels R, van Rossum E, Metzelthin S, Sipers W, Habets H, Hobma S, van den Heuvel W, de Witte L. A disability prevention programme for community-dwelling frail older persons. Clin Rehabil. 2011;25(11):963-974. doi: 10.1177/0269215511410728. 
